\title{
Noncommutative principal bundles through twist deformation
}

\author{
Paolo Aschieri ${ }^{1}$, Pierre Bieliavsky $^{2}$, Chiara Pagani $^{3}$, Alexander Schenkel $^{4}$ \\ ${ }^{1}$ Dipartimento di Scienze e Innovazione Tecnologica \\ and INFN Torino, \\ Università del Piemonte Orientale, Viale T. Michel 11, 15121 Alessandria, Italy. \\ (e-mail: aschieri@to.infn.it) \\ ${ }^{2}$ Institut de recherche en mathématique et physique, Université de Louvain, \\ Chemin du Cyclotron 2 bte L7.01.02, 1348 Louvain-la-Neuve, Belgium. \\ (e-mail: pierre.bieliavsky@uclouvain.be) \\ ${ }^{3}$ Mathematisches Institut, Georg-August-Universität Göttingen, \\ Bunsenstraße 3-5, 37073 Göttingen, Germany. \\ (e-mail: chiara.pagani@mathematik.uni-goettingen.de) \\ ${ }^{4}$ Fakultät für Mathematik, Universität Regensburg, 93040 Regensburg, Germany. \\ (e-mail: aschenkel83@gmail.com)
}

May 2016

\begin{abstract}
We construct noncommutative principal bundles deforming principal bundles with a Drinfeld twist (2-cocycle). If the twist is associated with the structure group then we have a deformation of the fibers. If the twist is associated with the automorphism group of the principal bundle, then we obtain noncommutative deformations of the base space as well. Combining the two twist deformations we obtain noncommutative principal bundles with both noncommutative fibers and base space. More in general, the natural isomorphisms proving the equivalence of a closed monoidal category of modules and its twist related one are used to obtain new Hopf-Galois extensions as twists of Hopf-Galois extensions. A sheaf approach is also considered, and examples presented.
\end{abstract}

Keywords: noncommutative geometry, noncommutative principal bundles, Hopf-Galois extensions, cocycle twisting

MSC 2010: $\quad$ 16T05, 16T15, 53D55, 81R50, 81R60, 18D35. 


\section{Contents}

1 Introduction

2 Background 5

2.1 Hopf-Galois extensions . . . . . . . . . . . . . . . . . . . . . . . . . . .

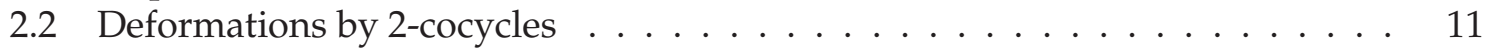

2.2.1 Hopf algebra 2-cocycles . . . . . . . . . . . . . . . 12

2.2.2 Twisting of right $H$-comodules . . . . . . . . . . . . . 13

2.2.3 Twisting of left K-comodules . . . . . . . . . . . . . . . . . . 16

2.2.4 Twisting of $(K, H)$-bicomodules . . . . . . . . . . . . 18

3 Twisting of Hopf-Galois extensions $\quad 20$

3.1 Deformation via a 2-cocycle on the structure Hopf algebra $H$. . . . . . . . . 21

3.2 Deformation via a 2-cocycle based on an external symmetry $K \ldots \ldots$. . . . 28

3.3 Combination of deformations . . . . . . . . . . . . . 35

4 Applications 37

4.1 Twisting quantum homogeneous spaces associated with quantum subgroups 37

4.2 Twisting sheaves of Hopf-Galois extensions . . . . . . . . . . . . . . 43

A Twists, 2-cocycles and untwisting 46

A.1 Drinfeld twists . . . . . . . . . . . . . . . . . . . . . . . . . . . .

A.2 Duality between twists and 2-cocycles . . . . . . . . . . . . . . . . . . . . . . .

A.3 Untwisting with 2 -cocycles . . . . . . . . . . . . . . 48

B Equivalence of closed monoidal categories and the 65 -map 49

C The twisted sheaf of the Hopf bundle over $S_{\theta}^{4}$ : top down approach 50

C.1 The spheres $S^{4}$ and $S_{\theta}^{4}$ as ringed spaces $\ldots \ldots \ldots \ldots \ldots$

\section{Introduction}

Given the relevance of Lie groups and principal bundles, the noncommutative analogues of these structures have been studied since the early days of noncommutative (NC) geometry, first examples being quantum groups and their coset spaces. The algebraic structure underlying NC principal bundles is that of Hopf-Galois extension, the structure group G being replaced by a Hopf algebra $H$ (e.g. that of functions on $G$, or more in general a neither commutative nor cocommutative Hopf algebra). Presently, in the literature, there are many examples of NC principal bundles, most of them can be understood as deformation quantization of classical principal bundles, see e.g. [9, 8]. Our NC geometry study of principal bundles is in this deformation quantization context, and specifically Drinfeld twist (or 2-cocycle) deformations [17, 16]. We provide a general theory where both the base space and the fibers are deformed, this allows to recover previously studied examples as particular cases, including a wide class of NC principal bundles on quantum coset spaces, as well as the NC instanton bundle on the $\theta$-sphere $S_{\theta}^{4}$ [14, 23, 24]. 
Drinfeld twist deformation is indeed a powerful method. It applies to any algebra $A$ that carries an action of a group (more in general a coaction of a Hopf algebra $K$ ). Given a twist on the group one first deforms the group in a quantum group and then canonically induces via its action a deformation of the algebra. Similarly, modules over algebras are twisted into modules over deformed algebras, in particular into NC vector bundles. This program has been successfully extended in [4] to the differential geometry of NC vector bundles. It has led to a theory of arbitrary (i.e., not necessary equivariant) connections on bimodules and on their tensor products that generalizes the notion of bimodule connection introduced in [28, 19]. The construction is categorical, and in particular commutative connections can be canonically quantized to NC connections. As sharpened in [5, 6] the categorical setting is that of closed monoidal categories.

In the next level of complexity after algebras and (bi)modules we find $(A, H)$-relative Hopf modules, i.e. (bi)modules with respect to an algebra $A$ and comodules with respect to an Hopf algebra $H$ (in particular we will be concerned with the example $A \otimes H$, that in the commutative case corresponds to the algebra of functions on the total space of a principal bundle tensored that on the structure group). They are the first objects of our interest because principality of NC bundles is bijectivity of a map (the so-called canonical map) between relative Hopf modules.

In this paper we thus first deform the category of $(A, H)$-relative Hopf modules by considering a twist associated with $H$ itself (this is the special degenerate case where $K=H$ ). Next we consider the case where there is a different Hopf algebra $K$ that coacts on $A$ and on $(A, H)$-relative Hopf modules, and study how to twist deform this category using twists on $K$, and then using twists both on $K$ and on $H$. Studying this category we are canonically led to twist deform classical principal bundles into NC principal bundles and more generally to prove that NC principal bundles are twisted into new NC principal bundles. The key point is to relate the canonical map between the twisted modules to the initial canonical map, so to deduce bijectivity of the first from bijectivity of the second. This is achieved via a set of isomorphisms that are explicitly constructed and have a categorical interpretation as components of natural isomorphisms.

Considering a twist on the "structure group" $H$ leads to a deformation of the fibers of the principal bundle; this result was also obtained in [27] with a different proof that disregards the natural categorical setting we are advocating. Considering a twist on "the external symmetry Hopf algebra" $K$ (classically associated with a subgroup of the automorphism group of the bundle) leads to a deformation of the base space. Combining twists on $H$ and on $K$ we obtain deformations of both the fibers and the base space.

The categorical context of relative Hopf modules and of their twist deformations we set up is furthermore used in order to prove that principal $H$-comodule algebras (i.e. HopfGalois extensions that admit the construction of associated vector bundles) are deformed into principal $\mathrm{H}$-comodule algebras (Corollary 3.19), here principality is captured by a linear map that is not in general $K$-equivariant and that has to be properly deformed. This deformation is explicitly given and shown to be related to the natural isomorphism proving the equivalence of the categories of Hopf algebra modules and of twisted Hopf algebra modules as closed monoidal categories. This same categorical context is relevant for planned further investigations in the geometry of NC principal bundles, in particular in the notion of gauge group and of principal connection. Indeed both gauge transformations and connections, as is the case for connections on NC vector bundles [4], will not in general be K-equivariant maps. 
Complementing the global description of principal G-bundles as G-manifolds with extra properties, there is the important local description based on trivial principal bundles and on transition functions. We therefore also present a local theory of twists deformations of NC principal bundles, based on a sheaf theoretic approach that complements the initial global approach. The explicit example of the $\theta$-sphere $S_{\theta}^{4}$ is detailed.

Finally we observe that the present study is mainly algebraic so that the examples treated are either in the context of formal deformation quantization, using Fréchet Hopf-Galois extensions on the ring $\mathbb{C}[[\hbar]]$ (cf. the main Example 3.24), or obtained via abelian Drinfeld twists associated with tori actions on algebraic varieties. However these latter NC algebras can be completed to $C^{*}$-algebras by the general deformation construction of Rieffel [32]; furthermore, also deformations of smooth manifolds based on nonabelian Drinfeld twists can be constructed nonformally [7]. It is then promising to combine these nonformal deformation techniques with the algebraic and categorical ones here developed in order to consider nonformal deformations of principal bundles. This is even more so because, contrary to the well established theory of NC vector bundles (consider for example finite projective $C^{*}$ modules over $C^{*}$-algebras), a general characterization of $\mathrm{NC}$ principal bundles beyond the algebraic level and in terms of NC topology is still missing. In particular we are interested in the wide class of nonformal NC principal bundles that could be obtained via twists based on an external symmetry Hopf algebra $K$. The present paper is also motivated by this program and can be seen as the first step toward its accomplishment.

The outline of the paper is the following: in $\$ 2.1$ we recall the basic definitions and results about Hopf-Galois extensions, while in \$2.2 we review some results from the theory of deformations of Hopf algebras and comodule algebras by 2-cocycles and extend them to the category of relative Hopf-modules, relevant to our study. The main results of the present paper are contained in $\$ 3$, in three successive subsections we study deformations of H-Hopf-Galois extensions by 2-cocycles on the structure group $H$ (\$3.1), on an external Hopf algebra $K$ of symmetries (\$3.2), and the combination of these deformations (\$3.3). In $\$ 4$ we apply the theory developed to deformations of quantum homogeneous spaces (\$4.1) and to encompass sheaves of Hopf-Galois extensions (\$4.2), providing also examples. Appendix $\mathrm{A}$ reviews the close relationship between the theory of 2-cocycles and that of Drinfeld twists, and Appendix B clarifies the relationship between one of our deformation maps (the 65 map) and the natural transformation which establishes that twisting may be regarded as an equivalence of closed monoidal categories. Appendix C presents a complementary study of the twisted sheaf describing the instanton bundle on $S_{\theta}^{4}$.

Acknowledgments: We would like to thank Tomasz Brzeziński, Lucio Cirio, Francesco D'Andrea, Rita Fioresi, Giovanni Landi, Zoran Škoda and Stefan Waldmann for useful comments and discussions. The research of P.B. and (in the initial stages of the project) C.P. was supported by the Belgian Scientific Policy under IAP grant DYGEST. A.S. was supported by a research fellowship of the Deutsche Forschungsgemeinschaft (DFG, Germany). The authors are members of the COST Action MP1405 QSPACE, supported by COST (European Cooperation in Science and Technology). P.A. is affiliated to INdAM, GNFM (Istituto Nazionale di Alta Matematica, Gruppo Nazionale di Fisica Matematica). 


\section{Background}

Notation: We work in the category of $\mathbb{K}$-modules, for $\mathbb{K}$ a fixed commutative ring with unit $1_{\mathbb{K}}$. We denote the tensor product over $\mathbb{K}$ just by $\otimes$. Morphisms of $\mathbb{K}$-modules are simply called $\mathbb{K}$-linear maps. In the following, all algebras are over $\mathbb{K}$ and assumed to be unital and associative. The product in an algebra $A$ is denoted by $m_{A}: A \otimes A \rightarrow A, a \otimes b \mapsto a b$ and the unit map by $\eta_{A}: \mathbb{K} \rightarrow A$, with $1_{A}:=\eta_{A}\left(1_{\mathbb{K}}\right)$ the unit element. Analogously all coalgebras are assumed to be over $\mathbb{K}$, counital and coassociative. We denote the coproduct and counit of a coalgebra $C$ by $\Delta_{C}: C \rightarrow C \otimes C$ and $\varepsilon_{C}: C \rightarrow \mathbb{K}$ respectively. We use the standard Sweedler notation for the coproduct: $\Delta_{C}(c)=c_{(1)} \otimes \mathcal{C}_{(2)}$ (sum understood), for all $c \in C$, and for iterations of it $\Delta_{C}^{n}=\left(\mathrm{id} \otimes \Delta_{C}\right) \circ \Delta_{C}^{n-1}: c \mapsto c_{(1)} \otimes c_{(2)} \otimes \cdots \otimes c_{(n+1)}, n>1$. We denote by $*$ the convolution product in the dual $\mathbb{K}$-module $C^{\prime}:=\operatorname{Hom}(C, \mathbb{K}),\left(f * f^{\prime}\right)(c):=f\left(c_{(1)}\right) f^{\prime}\left(c_{(2)}\right)$, for all $c \in C$, $f, f^{\prime} \in C^{\prime}$. Finally, for a Hopf algebra $H$, we denote by $S_{H}: H \rightarrow H$ its antipode. For all maps mentioned above we will omit the subscripts referring to the co/algebras involved when no risk of confusion can occur. Many of the examples presented will concern co/algebras equipped with an antilinear involution (*-structure); we will assume all maps therein to be compatible with the *-structure. To indicate an object $V$ in a category $C$ we frequently simply write $V \in C$. Finally, all monoidal categories appearing in this paper will have a trivial associator, hence we can unambiguously write $V_{1} \otimes V_{2} \otimes \cdots \otimes V_{n}$ for the tensor product of $n$ objects.

\subsection{Hopf-Galois extensions}

We briefly collect the algebraic preliminaries on Hopf-Galois extensions required for our work. Let $H$ be a bialgebra (or just a coalgebra). A right $H$-comodule is a $\mathbb{K}$-module $V$ with a $\mathbb{K}$-linear map $\delta^{V}: V \rightarrow V \otimes H$ (called a right $H$-coaction) such that

$$
(\mathrm{id} \otimes \Delta) \circ \delta^{V}=\left(\delta^{V} \otimes \mathrm{id}\right) \circ \delta^{V}, \quad(\mathrm{id} \otimes \varepsilon) \circ \delta^{V}=\mathrm{id} .
$$

The coaction on an element $v \in V$ is written in Sweedler notation as $\delta^{V}(v)=v_{(0)} \otimes v_{(1)}$ (sum understood). The right $H$-comodule properties (2.1) then read as, for all $v \in V$,

$$
\begin{aligned}
v_{(0)} \otimes\left(v_{(1)}\right)_{(1)} \otimes\left(v_{(1)}\right)_{(2)} & =\left(v_{(0)}\right)_{(0)} \otimes\left(v_{(0)}\right)_{(1)} \otimes v_{(1)}=: v_{(0)} \otimes v_{(1)} \otimes v_{(2)}, \\
v_{(0)} \varepsilon\left(v_{(1)}\right) & =v .
\end{aligned}
$$

We denote by $\mathcal{M}^{H}$ the category of right $H$-comodules, with the obvious definition of right $H$-comodule morphisms: a morphism between $V, W \in \mathcal{M}^{H}$ is a $\mathbb{K}$-linear map $\psi: V \rightarrow W$ which satisfies $\delta^{W} \circ \psi=(\psi \otimes \mathrm{id}) \circ \delta^{V}$ (H-equivariance condition). If $H$ is a bialgebra, then $\mathcal{M}^{H}$ is a monoidal category: given $V, W \in \mathcal{M}^{H}$, then the tensor product $V \otimes W$ is an object in $\mathcal{M}^{H}$ with the right $H$-coaction

$$
\begin{aligned}
\delta^{V \otimes W}: V \otimes W & \longrightarrow V \otimes W \otimes H, \\
v \otimes w & \longmapsto v_{(0)} \otimes w_{(0)} \otimes v_{(1)} w_{(1)} .
\end{aligned}
$$

The unit object in $\mathcal{M}^{H}$ is $\mathbb{K}$ together with the coaction given by the unit map of $H$, i.e., $\delta^{\mathbb{K}}:=\eta_{H}: \mathbb{K} \rightarrow \mathbb{K} \otimes H \simeq H$.

If $A$ is a right $H$-comodule and also an algebra it is natural to require the additional structures of product $m_{A}: A \otimes A \rightarrow A$ and unit $\eta_{A}: \mathbb{K} \rightarrow A$ to be morphisms in the category 
$\mathcal{M}^{H}$ (with $A \otimes A \in \mathcal{M}^{H}$ via $\delta^{A \otimes A}$ as above). Explicitly, a right $H$-comodule algebra $A$ is an algebra which is a right $H$-comodule and such that

$$
\delta^{A} \circ m_{A}=\left(m_{A} \otimes \mathrm{id}\right) \circ \delta^{A \otimes A}, \quad \delta^{A} \circ \eta_{A}=\left(\eta_{A} \otimes \mathrm{id}\right) \circ \delta^{\mathbb{K}} .
$$

This is equivalent to require the coaction $\delta^{A}: A \rightarrow A \otimes H$ to be a morphism of unital algebras (where $A \otimes H$ has the tensor product algebra structure), for all $a, a^{\prime} \in A$,

$$
\delta^{A}\left(a a^{\prime}\right)=\delta^{A}(a) \delta^{A}\left(a^{\prime}\right), \quad \delta^{A}\left(1_{A}\right)=1_{A} \otimes 1_{H} .
$$

A morphism between two right $H$-comodule algebras is a morphism in $\mathcal{M}^{H}$ which preserves products and units. We shall denote by $\mathcal{A}^{H}$ the category of right $H$-comodule algebras.

If $V$ is a right $H$-comodule and also a left $A$-module, where $A \in \mathcal{A}^{H}$, it is natural to require the $A$-module structure (or $A$-action) $\triangleright_{V}: A \otimes V \rightarrow V, a \otimes v \mapsto a \triangleright_{V} v$ to be a morphism in the category $\mathcal{M}^{H}$, i.e. $\delta^{V} \circ \triangleright_{V}=\left(\triangleright_{V} \otimes\right.$ id) $\circ \delta^{A \otimes V}$ (with $A \otimes V \in \mathcal{M}^{H}$ via $\delta^{A \otimes V}$ as above). We thus define the category of relative Hopf modules:

Definition 2.1. Let $H$ be a bialgebra and $A \in \mathcal{A}^{H}$. An $(A, H)$-relative Hopf module $V$ is a right $H$-comodule with a compatible left $A$-module structure, i.e. the left $A$-action $\triangleright_{V}$ is a morphism of $H$-comodules according to the following commutative diagram

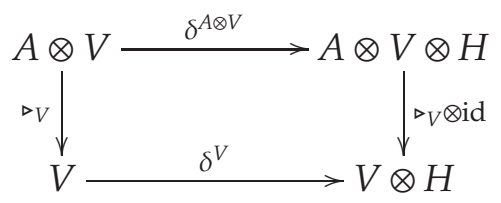

Explicitly, for all $a \in A$ and $v \in V$,

$$
\left(a \triangleright_{V} v\right)_{(0)} \otimes\left(a \triangleright_{V} v\right)_{(1)}=a_{(0)} \triangleright_{V} v_{(0)} \otimes a_{(1)} v_{(1)}
$$

A morphism of $(A, H)$-relative Hopf modules is a morphism of right $H$-comodules which is also a morphism of left $A$-modules. We denote by ${ }_{A} \mathcal{M}^{H}$ the category of $(A, H)$-relative Hopf modules.

Remark 2.2. If $V$ is a left $A$-module then $V \otimes H$ is a left $(A \otimes H)$-module via the left $(A \otimes H)$-action $(a \otimes h)\left(v \otimes h^{\prime}\right):=a \triangleright_{V} v \otimes h h^{\prime}$, for all $a \in A, v \in V, h, h^{\prime} \in H$. The commutativity of the diagram (2.6) is equivalent to

$$
\delta^{V}\left(a \triangleright_{V} v\right)=\delta^{A}(a) \delta^{V}(v),
$$

i.e., $\delta^{V}$ is a left $A$-module morphism, where the left $A$-action on $V \otimes H$ is via $\delta^{A}: A \rightarrow A \otimes H$ and the left $(A \otimes H)$-action above.

Analogously to Definition 2.1 we define the categories of relative Hopf modules $\mathcal{M}_{A}{ }^{H}$ and ${ }_{A} \mathcal{M}_{A}{ }^{H}$ :

Definition 2.3. Let $H$ be a bialgebra and $A \in \mathcal{A}^{H}$. 
(i) The objects in the category $\mathcal{M}_{A}{ }^{H}$ are right $H$-comodules with a compatible right $A$ module structure, i.e. $V$ is an object in $\mathcal{M}_{A}{ }^{H}$ if the right $A$-action $\triangleleft_{V}$ is a morphism of $H$-comodules according to the following commutative diagram

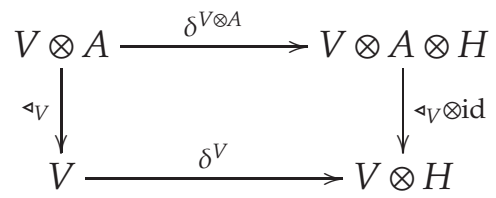

Explicitly, for all $a \in A$ and $v \in V$,

$$
\left(v \triangleleft_{V} a\right)_{(0)} \otimes\left(v \triangleleft_{V} a\right)_{(1)}=v_{(0)} \triangleleft_{V} a_{(0)} \otimes v_{(1)} a_{(1)} .
$$

The morphisms in the category $\mathcal{M}_{A}{ }^{H}$ are morphisms of right $H$-comodules which are also morphisms of right $A$-modules.

(ii) The objects in the category ${ }_{A} \mathcal{M}_{A}{ }^{H}$ are right $H$-comodules with a compatible $A$-bimodule structure, i.e. the commuting left and right $A$-actions satisfy respectively (2.6) and (2.9). The morphisms in the category ${ }_{A} \mathcal{M}_{A}{ }^{H}$ are morphisms of right $H$-comodules which are also morphisms of $A$-bimodules.

Given a left $A$-module $V$ and a $\mathbb{K}$-module $W$, the $\mathbb{K}$-module $V \otimes W$ is a left $A$-module with left action defined by $\triangleright_{V \otimes W}:=\triangleright_{V} \otimes$ id, i.e.

$$
\begin{aligned}
\triangleright_{V \otimes W}: A \otimes V \otimes W & \longrightarrow V \otimes W, \\
a \otimes v \otimes w & \longmapsto\left(a \triangleright_{V} v\right) \otimes w .
\end{aligned}
$$

Lemma 2.4. If $V \in{ }_{A} \mathcal{M}^{H}$ and $W \in \mathcal{M}^{H}$, then the right $H$-comodule $V \otimes W$ equipped with the left A-action given by (2.11) is an object in ${ }_{A} \mathcal{M}^{H}$.

Proof. We prove that the compatibility condition (2.7) between the left $A$-action $\triangleright_{V \otimes W}$ (see (2.11) ) and the right $H$-coaction $\delta^{V \otimes W}$ (see (2.3) ) is satisfied:

$$
\begin{aligned}
\left(a \triangleright_{V \otimes W}(v \otimes w)\right)_{(0)} \otimes\left(a \triangleright_{V \otimes W}(v \otimes w)\right)_{(1)} & =\left(\left(a \triangleright_{V} v\right) \otimes w\right)_{(0)} \otimes\left(\left(a \triangleright_{V} v\right) \otimes w\right)_{(1)} \\
& =\left(a \triangleright_{V} v\right)_{(0)} \otimes w_{(0)} \otimes\left(a \triangleright_{V} v\right)_{(1)} w_{(1)} \\
& =\left(a_{(0)} \triangleright_{V} v_{(0)}\right) \otimes w_{(0)} \otimes a_{(1)} v_{(1)} w_{(1)} \\
& =a_{(0)} \triangleright_{V \otimes W}\left(v_{(0)} \otimes w_{(0)}\right) \otimes a_{(1)} v_{(1)} w_{(1)} \\
& =a_{(0)} \triangleright_{V \otimes W}(v \otimes w)_{(0)} \otimes a_{(1)}(v \otimes w)_{(1)},
\end{aligned}
$$

where in the third passage we have used that (2.7) holds for the left $A$-action $\triangleright_{V}$ and the right $H$-coaction $\delta^{V}$.

Remark 2.5. More abstractly, Lemma 2.4 states that ${ }_{A} \mathcal{M}^{H}$ is a (right) module category over the monoidal category $\mathcal{M}^{H}$, see e.g. [29]. Indeed, we have a bifunctor (denoted with abuse of notation also by $\otimes) \otimes:{ }_{A} \mathcal{M}^{H} \times \mathcal{M}^{H} \rightarrow{ }_{A} \mathcal{M}^{H}$ which assigns to an object $(V, W) \in{ }_{A} \mathcal{M}^{H} \times \mathcal{M}^{H}$ the object $V \otimes W \in{ }_{A} \mathcal{M}^{H}$ constructed in Lemma 2.4 and to a morphism $\left(f: V_{1} \rightarrow V_{2}, g\right.$ : $\left.W_{1} \rightarrow W_{2}\right)$ in ${ }_{A} \mathcal{M}^{H} \times \mathcal{M}^{H}$ the ${ }_{A} \mathcal{M}^{H}$-morphism $f \otimes g: V_{1} \otimes W_{1} \rightarrow V_{2} \otimes W_{2}, v \otimes w \mapsto f(v) \otimes g(w)$. (It is easy to check that $f \otimes g$ is a morphism of left $A$-modules). 
Analogously, given a right $A$-module $V$ and a $\mathbb{K}$-module $W$, the $\mathbb{K}$-module $W \otimes V$ is a right $A$-module with right action defined by $\triangleleft_{W \otimes V}:=\mathrm{id} \otimes \triangleleft_{V}$. We omit the proof of the corresponding

Lemma 2.6. If $V \in \mathcal{M}_{A}{ }^{H}$ and $W \in \mathcal{M}^{H}$, then the right $H$-comodule $W \otimes V$ equipped with the right A-action given by $\triangleleft_{W \otimes V}:=\mathrm{id} \otimes \triangleleft_{V}$ is an object in $\mathcal{M}_{A}{ }^{H}$.

Remark 2.7. Analogously to Remark 2.5, Lemma 2.6 states that $\mathcal{M}_{A}{ }^{H}$ is a (left) module category over the monoidal category $\mathcal{M}^{H}$. We denote again with an abuse of notation the corresponding bifunctor simply by $\otimes: \mathcal{M}^{H} \times \mathcal{M}_{A}^{H} \rightarrow \mathcal{M}_{A}^{H}$.

Remark 2.8. Combining Remark 2.5 and Remark 2.7 we have a bifunctor (denoted again by the same symbol) $\otimes:{ }_{A} \mathcal{M}^{H} \times \mathcal{M}_{A}{ }^{H} \rightarrow{ }_{A} \mathcal{M}_{A}{ }^{H}$. Precomposing this functor with the forgetful functor ${ }_{A} \mathcal{M}_{A}{ }^{H} \times{ }_{A} \mathcal{M}_{A}{ }^{H} \rightarrow{ }_{A} \mathcal{M}^{H} \times \mathcal{M}_{A}{ }^{H}$ we obtain another bifunctor (denoted once more by the same symbol) $\otimes:{ }_{A} \mathcal{M}_{A}{ }^{H} \times{ }_{A} \mathcal{M}_{A}{ }^{H} \rightarrow{ }_{A} \mathcal{M}_{A}{ }^{H}$. Notice that this bifunctor satisfies the associativity constraint, however it does not structure ${ }_{A} \mathcal{M}_{A}{ }^{H}$ as a monoidal category since there exists no unit object $I \in{ }_{A} \mathcal{M}_{A}{ }^{H}$. We therefore consider the tensor product over the algebra $A, \otimes_{A}:{ }_{A} \mathcal{M}_{A}{ }^{H} \times{ }_{A} \mathcal{M}_{A}{ }^{H} \rightarrow{ }_{A} \mathcal{M}_{A}{ }^{H}$ (obtained via the standard quotient procedure) that turns ${ }_{A} \mathcal{M}_{A}{ }^{H}$ into a monoidal category with unit object $A \in{ }_{A} \mathcal{M}_{A}{ }^{H}$.

In the following $H$ will be assumed to be a Hopf algebra.

Definition 2.9. Let $H$ be a Hopf algebra and $A \in \mathcal{A}^{H}$. Let $B \subseteq A$ be the subalgebra of coinvariants, i.e.

$$
B:=A^{c o H}=\left\{b \in A \mid \delta^{A}(b)=b \otimes 1_{H}\right\}
$$

The map

$$
\begin{aligned}
\chi:=(m \otimes \mathrm{id}) \circ\left(\mathrm{id} \otimes_{B} \delta^{A}\right): A \otimes_{B} A & \longrightarrow A \otimes H, \\
a \otimes_{B} a^{\prime} & \longmapsto a a_{(0)}^{\prime} \otimes a_{(1)}^{\prime}
\end{aligned}
$$

is called the canonical map. The extension $B \subseteq A$ is called an $H$-Hopf-Galois extension provided the canonical map is bijective.

The notion of Hopf-Galois extensions in this general context of (not necessarily commutative) algebras appeared in [22]. It generalizes the classical notion of Galois field extensions and with a noncommutative flavor it can be viewed as encoding the data of a principal bundle. We refer the reader to the references [26], [10, Part VII] and examples therein. See also Example 2.13 below.

In the special case when $A$ is commutative (and hence also $B \subseteq A$ is commutative), then $A \otimes_{B} A$ is an algebra and the canonical map $\chi$ is an algebra morphism. In general however $A$ is noncommutative and also $B$ is not contained in the center of $A$, so $A \otimes_{B} A$ does not even inherit an algebra structure. As we shall now show, in the general case the canonical map $\chi$ is a morphism in the category of relative Hopf modules ${ }_{A} \mathcal{M}_{A}{ }^{H}$.

The tensor product $A \otimes A$ is an object in ${ }_{A} \mathcal{M}_{A}{ }^{H}$ because of Lemma 2.4 (take $V=A$ with left $A$-action given by the product in $A$ and $W=A$ ) and of Lemma 2.6 (take $V=A$ with right $A$-action given by the product in $A$ and $W=A$ ); the compatibility between the left and the right $A$-actions is immediate: $c\left(\left(a \otimes a^{\prime}\right) c^{\prime}\right)=c a \otimes a^{\prime} c^{\prime}=\left(c\left(a \otimes a^{\prime}\right)\right) c^{\prime}$, for all $a, a^{\prime}, c, c^{\prime} \in A$. 
The right $H$-coaction $\delta^{A \otimes A}: A \otimes A \rightarrow A \otimes A \otimes H$ descends to the quotient $A \otimes_{B} A$ because $B \subseteq A$ is the subalgebra of $H$-coinvariants. We denote the induced right $H$-coaction by $\delta^{A \otimes_{B} A}$. The left and right $A$-actions on $A \otimes A$ also canonically descend to the quotient $A \otimes_{B} A$, hence they are compatible with the right $H$-comodule structure (cf. (2.6) and (2.9)) and therefore $A \otimes_{B} A \in{ }_{A} \mathcal{M}_{A}^{H}$.

The tensor product $A \otimes H$ is also an object in ${ }_{A} \mathcal{M}_{A}{ }^{H}$. First we regard the Hopf algebra $H$ as the right $H$-comodule $\underline{H}$ defined to be the $\mathbb{K}$-module $H$ with the right adjoint $H$-coaction

$$
\delta \underline{H}=\mathrm{Ad}: \underline{H} \longrightarrow \underline{H} \otimes H, h \longmapsto h_{(2)} \otimes S\left(h_{(1)}\right) h_{(3)} .
$$

(The notation $\underline{H}$ is in order to distinguish this structure from the Hopf algebra structure). Then, since $A \in{ }_{A} \mathcal{M}^{H}$ and $\underline{H} \in \mathcal{M}^{H}$, Lemma 2.4 implies that $A \otimes \underline{H} \in{ }_{A} \mathcal{M}^{H}$. Explicitly the right $H$-coaction $\delta^{A \otimes \underline{H}}: A \otimes \underline{H} \rightarrow A \otimes \underline{H} \otimes H$ is given by 1 (cf. (2.3) ), for all $a \in A, h \in \underline{H}$,

$$
\delta A \underline{H}(a \otimes h)=a_{(0)} \otimes h_{(2)} \otimes a_{(1)} S\left(h_{(1)}\right) h_{(3)} \in A \otimes \underline{H} \otimes H .
$$

Finally, $A \otimes \underline{H}$ is a right $A$-module with the action

$$
\begin{aligned}
\triangleleft_{A \otimes \underline{H}}: A \otimes \underline{H} \otimes A & \longrightarrow A \otimes \underline{H}, \\
a \otimes h \otimes c & \longmapsto a c_{(0)} \otimes h c_{(1)} .
\end{aligned}
$$

This right $A$-action is easily seen to be a morphism in $\mathcal{M}^{H}$, indeed the diagram

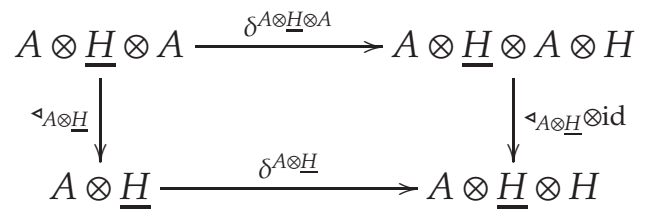

is commutative. Here, according to (2.3), $\delta^{A \otimes \underline{H} \otimes A}(a \otimes h \otimes c)=a_{(0)} \otimes h_{(2)} \otimes c_{(0)} \otimes a_{(1)} S\left(h_{(1)}\right) h_{(3)} c_{(1)}$, for all $a, c \in A, h \in \underline{H}$. This shows that $A \otimes \underline{H} \in \mathcal{M}_{A}^{H}$. Since the left and right $A$-actions commute we conclude that $A \otimes \underline{H} \in{ }_{A} \mathcal{M}_{A}{ }^{H}$.

Proposition 2.10. The canonical map $\chi=(m \otimes \mathrm{id}) \circ\left(\mathrm{id} \otimes_{B} \delta^{A}\right): A \otimes_{B} A \rightarrow A \otimes \underline{H}$ is a morphism in ${ }_{A} \mathcal{M}_{A}{ }^{H}$ with respect to the ${ }_{A} \mathcal{M}_{A}{ }^{H}$-structures on $A \otimes_{B} A$ and $A \otimes \underline{H}$ described above.

Proof. We show that the canonical map is a morphism of right $H$-comodules, for all $a, a^{\prime} \in A$,

$$
\begin{aligned}
\delta^{A \otimes \underline{H}}\left(\chi\left(a \otimes_{B} a^{\prime}\right)\right) & =\delta^{A \otimes \underline{H}}\left(a a_{(0)}^{\prime} \otimes a_{(1)}^{\prime}\right)=a_{(0)} a_{(0)}^{\prime} \otimes a_{(3)}^{\prime} \otimes a_{(1)} a_{(1)}^{\prime} S\left(a_{(2)}^{\prime}\right) a_{(4)}^{\prime} \\
& =a_{(0)} a_{(0)}^{\prime} \otimes a_{(1)}^{\prime} \otimes a_{(1)} a_{(2)}^{\prime}=(\chi \otimes \mathrm{id})\left(\left(a_{(0)} \otimes_{B} a_{(0)}^{\prime}\right) \otimes a_{(1)} a_{(1)}^{\prime}\right) \\
& =(\chi \otimes \mathrm{id})\left(\delta^{A \otimes_{B} A}\left(a \otimes_{B} a^{\prime}\right)\right) .
\end{aligned}
$$

It is immediate to see that $\chi$ is a morphism of left and right $A$-modules.

\footnotetext{
${ }^{1}$ Similarly on the tensor product $A \otimes H$ we also have the $H$-comodule structure $\delta^{A \otimes H}$ induced by the right regular coaction (coproduct) of $H$. Notice that if $A$ is isomorphic to the $H$-comodule $B \otimes H$ with right coaction $\mathrm{id}_{B} \otimes \Delta$ (hence in particular if $A$ is cleft, see page 11), then the $H$-comodules $\left(A \otimes H, \delta^{A \otimes H}\right)$ and $\left(A \otimes \underline{H}, \delta^{A \otimes}\right)$ are isomorphic. The isomorphism is given by $A \otimes H \rightarrow A \otimes \underline{H},(a \otimes h) \mapsto\left(a h_{1} \otimes h_{2}\right)$, with inverse $A \otimes \underline{H} \rightarrow A \otimes H$, $(a \otimes h) \mapsto\left(a S\left(h_{1}\right) \otimes h_{2}\right)$, where $a h$ indicates the action of $H$ on $A \simeq B \otimes H$ given by right multiplication.
} 
Example 2.11. Let $A=H$ be the right $H$-comodule algebra with right $H$-coaction given by the coproduct $\Delta$. Since $(\varepsilon \otimes \mathrm{id}) \Delta(h)=h$, for all $h \in H$, we have $H^{c o H} \simeq \mathbb{K}$. The canonical map $\chi: H \otimes H \rightarrow H \otimes \underline{H}$ is an isomorphism with inverse $\chi^{-1}\left(h \otimes h^{\prime}\right)=h S\left(h^{\prime}{ }_{(1)}\right) \otimes h^{\prime}{ }_{(2)}$, for all $h \in H$ and $h^{\prime} \in \underline{H}$. Hence, $\mathbb{K} \subseteq H$ is an $H$-Hopf-Galois extension. Notice that $S(h)=(\operatorname{id} \otimes \varepsilon) \chi^{-1}(1 \otimes h)$, for all $h \in H$; actually a bialgebra $H$ is a Hopf algebra if and only if $\chi: H \otimes H \rightarrow H \otimes \underline{H}$ is an isomorphism.

Example 2.12. Let $B$ be an algebra with trivial right $H$-coaction, i.e. $\delta^{B}(b)=b \otimes 1$ for all $b \in B$. Let, as in the previous example, $H$ be the right $H$-comodule algebra with the coaction given by the coproduct $\Delta$. Then $A:=B \otimes H$ is a right $H$-comodule algebra (with the usual tensor product algebra and right $H$-comodule structure). We have $A^{c o H} \simeq B$ and $\chi:(B \otimes H) \otimes_{B}(B \otimes H) \simeq B \otimes H \otimes H \rightarrow B \otimes H \otimes \underline{H}, b \otimes h \otimes h^{\prime} \mapsto b \otimes h h_{(1)}^{\prime} \otimes h_{(2)}^{\prime}$ is easily seen to be invertible; hence $B \subseteq A$ is an $H$-Hopf-Galois extension.

Example 2.13. Let $G$ be a Lie group, $M$ a manifold and $\pi: P \rightarrow M$ a principal $G$-bundle over $M$ with right $G$-action denoted by $r_{P}: P \times G \rightarrow P,(p, g) \mapsto p g$. (All manifolds here are assumed to be finite-dimensional and second countable). We assign to the total space $P$ its space of smooth functions $C^{\infty}(P)$ and recall that it is a (nuclear) Fréchet space with respect to the usual $C^{\infty}$-topology. Even more, the Fréchet space $A=C^{\infty}(P)$ is a unital Fréchet algebra with (continuous) product $m:=\operatorname{diag}_{P}^{*}: A \widehat{\otimes} A \rightarrow A$ and unit $\eta:=\mathrm{t}_{P}^{*}: \mathbb{K} \rightarrow A$. Here $A \widehat{\otimes} A \simeq C^{\infty}(P \times P)$ denotes the completed tensor product and the product and unit are defined as the pull-back on functions of the diagonal map $\operatorname{diag}_{P}: P \rightarrow P \times P$ and the terminal map $\mathrm{t}_{P}: P \rightarrow$ pt to a point. Similarly, $B=C^{\infty}(M)$ is a Fréchet algebra and $H=C^{\infty}(G)$ is a Fréchet Hopf algebra with co-structures and antipode defined by the pull-backs of the Lie group structures on $G$. (In a Fréchet Hopf algebra also the antipode, counit and coproduct $\Delta: H \rightarrow H \widehat{\otimes} H$ are continuous maps). The right $G$-action $r_{P}: P \times G \rightarrow P$ induces the structure of a Fréchet right $H$-comodule algebra on $A$ and we denote the (continuous) right $H$-coaction by $\delta^{A}:=r_{P}^{*}: A \rightarrow A \widehat{\otimes} H$. The $H$-coinvariant subalgebra is $A^{\mathrm{coH}}=C^{\infty}(P / G)$ and $A^{\mathrm{co} H} \simeq B=C^{\infty}(M)$ is the pull-back of the isomorphism $M \simeq P / G$ of the principal $G$-bundle $P \rightarrow M$. The canonical map in the present case may be obtained by considering the pull-back of the smooth map

$$
P \times G \longrightarrow P \times_{M} P,(p, g) \longmapsto(p, p g),
$$

where $P \times_{M} P:=\{(p, q) \in P \times P \mid \pi(p)=\pi(q)\}$ is the fibered product. This map is an isomorphism of right $G$-spaces, because the $G$-action on the fibers of $P$ is free and transitive. It follows that the canonical map 2 $\chi: A \widehat{\otimes}_{B} A \rightarrow A \widehat{\otimes} \underline{H}$ is an ${ }_{A} \mathcal{M}_{A}{ }^{H}$-isomorphism, hence $B \subseteq A$ is a Fréchet $H$-Hopf-Galois extension.

The previous two examples correspond to (algebraic versions of) the principal G-bundle $G \rightarrow$ pt over a point and to the trivial principal $G$-bundle $M \times G \rightarrow M$ over $M$.

An $H$-Hopf-Galois extension $B:=A^{c o H} \subseteq A$ is said to have the normal basis property if there exists an isomorphism $A \simeq B \otimes H$ of left $B$-modules and right $H$-comodules (where $B \otimes H$ is a left $B$-module via $m_{B} \otimes$ id and a right $H$-comodule via id $\otimes \Delta$, cf. Example 2.12).

\footnotetext{
2 The topological tensor product over $B$ is defined as follows: Consider the two parallel continuous linear maps $m \widehat{\otimes}$ id and id $\widehat{\otimes} m$ from $A \widehat{\otimes} B \widehat{\otimes} A$ to $A \widehat{\otimes} A$, which describe the right and respectively left $B$-action on $A$. We set $A \widehat{\otimes}_{B} A:=A \widehat{\otimes} A / \overline{\operatorname{Im}(m \widehat{\otimes} \mathrm{id}-\mathrm{id} \widehat{\otimes} m)}$, where - denotes the closure in the Fréchet space $A \widehat{\otimes} A$. Notice that $A \widehat{\otimes}_{B} A \simeq C^{\infty}\left(P \times_{M} P\right)$.
} 
This condition captures the algebraic aspect of triviality of a principal bundle. We recall that the normal basis property is equivalent to the existence of a convolution invertible map $j: H \rightarrow A$ (called cleaving map) that is a right $H$-comodule morphism, i.e.

$$
\delta^{A} \circ j=(j \otimes \mathrm{id}) \circ \Delta .
$$

A comodule algebra $A$ for which there exists a convolution invertible morphism of $H$ comodules $j: H \rightarrow A$ is called a cleft extension of $A^{c o H}$. Given an isomorphism $\theta: B \otimes H \rightarrow A$ of left $B$-modules and right $H$-comodules, then a cleaving map $j: H \rightarrow A$ and its convolution inverse $\bar{j}: H \rightarrow A$ are determined by

$$
\begin{aligned}
& j: H \longrightarrow A, h \longmapsto \theta(1 \otimes h), \\
& \bar{j}: H \longrightarrow A, h \longmapsto(\mathrm{id} \otimes \varepsilon) \circ\left(\mathrm{id} \otimes_{B} \theta^{-1}\right) \circ \chi^{-1}(1 \otimes h) .
\end{aligned}
$$

(In order to prove that $j * \bar{j}=\eta_{A} \circ \varepsilon$ use $A$-linearity of $\chi^{-1}$, then that $\theta$ is an $H$-comodule map and then recall the definition of $\chi$. In order to prove that $\bar{j} * j=\eta_{A} \circ \varepsilon$ it is convenient to set $\chi^{-1}(h)=h^{<1>} \otimes h^{<2>}$ for all $h \in H$, then use $\left(\chi^{-1} \otimes \mathrm{id}\right)(\mathrm{id} \otimes \Delta)=\left(\mathrm{id} \otimes \delta^{A}\right) \chi^{-1}$, observe that since $\theta$ is an $H$-comodule map so is $\theta^{-1}$ and hence (id $\left.\otimes \varepsilon \otimes \mathrm{id}\right)\left(\theta^{-1} \otimes \mathrm{id}\right) \delta^{A}=\theta^{-1}$, and that, due to left $B$-linearity of $\theta$, (id $\otimes \varepsilon)\left(\theta^{-1}\left(a_{(0)}\right) \theta\left(1 \otimes a_{(1)}\right)=a\right.$ for all $a \in A$. See e.g. [26, Theorem 8.2.4]).

To conclude this subsection, let us recall the definition of principal comodule algebra which, as it is the case for principal bundles, allows for the construction of associated vector bundles (i.e. associated finitely generated and projective $B$-modules). Among the equivalent formulations we consider the one here below [15] (see also [10, Part VII, §6.3 and §6.4]) because it will be easily seen to be preserved by twist deformations.

Definition 2.14. Let $H$ be a Hopf algebra with invertible antipode over a field $\mathbb{K}$. A principal $H$-comodule algebra is an object $A \in \mathcal{A}^{H}$ such that $B:=A^{c o H} \subseteq A$ is an $H$-Hopf-Galois extension and $A$ is equivariantly projective as a left $B$-module, i.e. there exists a left $B$-module and right $H$-comodule morphism $s: A \rightarrow B \otimes A$ that is a section of the (restricted) product $m: B \otimes A \rightarrow A$, i.e. such that $m \circ s=\mathrm{id}_{A}$.

In particular if $H$ is a Hopf algebra with bijective antipode over a field, the condition of equivariant projectivity of $A$ is equivalent to that of faithful flatness of $A$ [33]. Moreover, by the Theorem of characterization of faithfully flat extensions [34], if $H$ is cosemisimple and has a bijective antipode, then surjectivity of the canonical map is sufficient to prove the principality of $A$.

\subsection{Deformations by 2-cocycles}

We review some results from the theory of deformations of Hopf algebras $H$ and their comodule (co)algebras by 2-cocycles $\gamma: H \otimes H \rightarrow \mathbb{K}$. The notion of 2-cocycle is dual to that of Drinfeld twist. In Appendix $\mathrm{A}$ we detail this duality for the reader more familiar with the Drinfeld twist notation. We omit some of the proofs of standard results, see e.g. [16] and also [21, §10.2], or, in the dual Drinfeld twist picture, [25, §2.3]. We also extend results from the category of $\mathrm{H}$-comodules to those of relative Hopf (co)modules and bicomodules (cf. Proposition 2.21, Proposition 2.25, and Proposition 2.27) which will be relevant to our construction in 83 . 


\subsubsection{Hopf algebra 2-cocycles}

Let $H$ be a Hopf algebra and recall that $H \otimes H$ is canonically a coalgebra with coproduct $\Delta_{H \otimes H}(h \otimes k)=h_{(1)} \otimes k_{(1)} \otimes h_{(2)} \otimes k_{(2)}$ and counit $\varepsilon_{H \otimes H}(h \otimes k)=\varepsilon(h) \varepsilon(k)$, for all $h, k \in H$. In particular, we can consider the convolution product of $\mathbb{K}$-linear maps $H \otimes H \rightarrow \mathbb{K}$.

Definition 2.15. A $\mathbb{K}$-linear map $\gamma: H \otimes H \rightarrow \mathbb{K}$ is called a convolution invertible, unital 2-cocycle on $H$, or simply a 2-cocycle, provided $\gamma$ is convolution invertible, unital, i.e. $\gamma(h \otimes 1)=\varepsilon(h)=\gamma(1 \otimes h)$, for all $h \in H$, and satisfies the 2-cocycle condition

$$
\gamma\left(g_{(1)} \otimes h_{(1)}\right) \gamma\left(g_{(2)} h_{(2)} \otimes k\right)=\gamma\left(h_{(1)} \otimes k_{(1)}\right) \gamma\left(g \otimes h_{(2)} k_{(2)}\right),
$$

for all $g, h, k \in H$.

The following lemma is easily proven. The stated equalities will be used for computations in the next sections.

Lemma 2.16. Let $\gamma: H \otimes H \rightarrow \mathbb{K}$ be a convolution invertible $\mathbb{K}$-linear map, with inverse denoted by $\bar{\gamma}: H \otimes H \rightarrow \mathbb{K}$. Then the following are equivalent:

(i) $\gamma$ satisfies (2.21)

(ii) $\bar{\gamma}\left(g_{(1)} h_{(1)} \otimes k\right) \bar{\gamma}\left(g_{(2)} \otimes h_{(2)}\right)=\bar{\gamma}\left(g \otimes h_{(1)} k_{(1)}\right) \bar{\gamma}\left(h_{(2)} \otimes k_{(2)}\right)$, for all $g, h, k \in H$

(iii) $\gamma\left(g_{(1)} h_{(1)} \otimes k_{(1)}\right) \bar{\gamma}\left(g_{(2)} \otimes h_{(2)} k_{(2)}\right)=\bar{\gamma}\left(g \otimes h_{(1)}\right) \gamma\left(h_{(2)} \otimes k\right)$, for all $g, h, k \in H$

(iv) $\gamma\left(g_{(1)} \otimes h_{(1)} k_{(1)}\right) \bar{\gamma}\left(g_{(2)} h_{(2)} \otimes k_{(2)}\right)=\gamma\left(g \otimes h_{(2)}\right) \bar{\gamma}\left(h_{(1)} \otimes k\right)$, for all $g, h, k \in H$

Given a 2-cocycle $\gamma$, with the help of (iii) and (iv), it is possible to prove that the maps

$$
\begin{aligned}
& u_{\gamma}: H \longrightarrow \mathbb{K}, h \longmapsto \gamma\left(h_{(1)} \otimes S\left(h_{(2)}\right)\right), \\
& \bar{u}_{\gamma}: H \longrightarrow \mathbb{K}, h \longmapsto \bar{\gamma}\left(S\left(h_{(1)}\right) \otimes h_{(2)}\right),
\end{aligned}
$$

are the convolution inverse of each other.

Proposition 2.17. Let $\gamma: H \otimes H \rightarrow \mathbb{K}$ be a 2-cocycle. Then

$$
m_{\gamma}(h \otimes k):=h \cdot \gamma k:=\gamma\left(h_{(1)} \otimes k_{(1)}\right) h_{(2)} k_{(2)} \bar{\gamma}\left(h_{(3)} \otimes k_{(3)}\right),
$$

for all $h, k \in H$, defines a new associative product on (the $\mathbb{K}$-module underlying) $H$. The resulting algebra $H_{\gamma}:=\left(H, m_{\gamma}, 1_{H}\right)$ is a Hopf algebra when endowed with the unchanged coproduct $\Delta$ and counit $\varepsilon$ and with the new antipode $S_{\gamma}:=u_{\gamma} * S * \bar{u}_{\gamma}$. We call $H_{\gamma}$ the twisted Hopf algebra of $H$ by $\gamma$.

Remark 2.18. The twisted Hopf algebra $H_{\gamma}$ can be 'untwisted' by using the convolution inverse $\bar{\gamma}: H \otimes H \rightarrow \mathbb{K}$; indeed, $\bar{\gamma}$ is a 2-cocycle for $H_{\gamma}$ and the twisted Hopf algebra of $H_{\gamma}$ by $\bar{\gamma}$ is isomorphic to $H$ via the identity map (see Corollary A.4). 


\subsubsection{Twisting of right $H$-comodules}

The deformation of a Hopf algebra $H$ by a 2-cocycle $\gamma: H \otimes H \rightarrow \mathbb{K}$ affects also the category $\mathcal{M}^{H}$ of right $H$-comodules. Indeed, if $V \in \mathcal{M}^{H}$ with coaction $\delta^{V}: V \rightarrow V \otimes H$, then $V$ with the same coaction, but now thought of as a map with values in $V \otimes H_{\gamma}$, is a right $H_{\gamma}$-comodule. This is evident from the fact that the comodule condition (cf. (2.1) ) only involves the coalgebra structure of $H$, and $H_{\gamma}$ coincides with $H$ as a coalgebra. When thinking of $V$ as an object in $\mathcal{M}^{H_{\gamma}}$ we will denote it by $V_{\gamma}$ and the coaction by $\delta^{V_{\gamma}}: V_{\gamma} \rightarrow V_{\gamma} \otimes H_{\gamma}$. Moreover, any morphism $\psi: V \rightarrow W$ in $\mathcal{M}^{H}$ can be thought as a morphism $\psi: V_{\gamma} \rightarrow W_{\gamma}$ in $\mathcal{M}^{H_{\gamma}}$; indeed, $H$-equivariance of $\psi: V \rightarrow W$ implies $H_{\gamma}$-equivariance of $\psi: V_{\gamma} \rightarrow W_{\gamma}$ since by construction the right $H$-coaction in $V$ agrees with the right $H_{\gamma}$-coaction in $V_{\gamma}$. Hence we have a functor

$$
\Gamma: \mathcal{M}^{H} \rightarrow \mathcal{M}^{H_{\gamma}}
$$

defined by $\Gamma(V):=V_{\gamma}$ and $\Gamma(\psi):=\psi: V_{\gamma} \rightarrow W_{\gamma}$. Furthermore this functor $\Gamma$ induces an equivalence of categories because we can use the convolution inverse $\bar{\gamma}$ in order to twist back $H_{\gamma}$ to $\left(H_{\gamma}\right)_{\bar{\gamma}}=H$ and $V_{\gamma}$ to $\left(V_{\gamma}\right)_{\bar{\gamma}}=V$.

The equivalence between the categories $\mathcal{M}^{H}$ and $\mathcal{M}^{H_{\gamma}}$ extends to their monoidal structure. We denote by $\left(\mathcal{M}^{H_{\gamma}}, \otimes^{\gamma}\right)$ the monoidal category corresponding to the Hopf algebra $H_{\gamma}$. Explicitly, for all objects $V_{\gamma}, W_{\gamma} \in \mathcal{M}^{H_{\gamma}}$ (with coactions $\delta^{V_{\gamma}}: V_{\gamma} \rightarrow V_{\gamma} \otimes H_{\gamma}$ and $\delta^{W_{\gamma}}: W_{\gamma} \rightarrow$ $\left.W_{\gamma} \otimes H_{\gamma}\right)$, the right $H^{\gamma}$-coaction on $V_{\gamma} \otimes^{\gamma} W_{\gamma}$, according to (2.3), is given by

$$
\begin{aligned}
\delta^{V_{\gamma} \otimes^{\gamma} W_{\gamma}}: V_{\gamma} \otimes^{\gamma} W_{\gamma} & \longrightarrow V_{\gamma} \otimes^{\gamma} W_{\gamma} \otimes H_{\gamma}, \\
v \otimes^{\gamma} w & \longmapsto v_{(0)} \otimes^{\gamma} w_{(0)} \otimes v_{(1)} \cdot \gamma w_{(1)},
\end{aligned}
$$

Theorem 2.19. The functor $\Gamma: \mathcal{M}^{H} \rightarrow \mathcal{M}^{H_{\gamma}}$ induces an equivalence between the monoidal categories $\left(\mathcal{M}^{H}, \otimes\right)$ and $\left(\mathcal{M}^{H_{\gamma}}, \otimes^{\gamma}\right)$. The natural isomorphism $\varphi: \otimes^{\gamma} \circ(\Gamma \times \Gamma) \Rightarrow \Gamma \circ \otimes$ is given by the $\mathcal{M}^{H_{\gamma_{-}}}$ isomorphisms

$$
\begin{aligned}
\varphi_{V, W}: V_{\gamma} \otimes^{\gamma} W_{\gamma} & \longrightarrow(V \otimes W)_{\gamma} \\
v \otimes^{\gamma} w & \longmapsto v_{(0)} \otimes w_{(0)} \bar{\gamma}\left(v_{(1)} \otimes w_{(1)}\right),
\end{aligned}
$$

for all objects $V, W \in \mathcal{M}^{H}$.

Proof. The invertibility of $\varphi_{V, W}$ follows immediately from the invertibility of the cocycle $\gamma$. The fact that it is a morphism in the category $\mathcal{M}^{H_{\gamma}}$ is easily shown as follows:

$$
\begin{aligned}
&\left(\varphi_{V, W} \otimes \mathrm{id}\right)\left(\delta^{V_{\gamma} \otimes{ }^{\gamma} W_{\gamma}}\left(v \otimes \otimes^{\gamma} w\right)\right)=v_{(0)} \otimes w_{(0)} \bar{\gamma}\left(v_{(1)} \otimes w_{(1)}\right) \otimes \gamma\left(v_{(2)} \otimes w_{(2)}\right) v_{(3)} w_{(3)} \bar{\gamma}\left(v_{(4)} \otimes w_{(4)}\right) \\
&=v_{(0)} \otimes w_{(0)} \otimes v_{(1)} w_{(1)} \bar{\gamma}\left(v_{(2)} \otimes w_{(2)}\right) \\
&=\delta^{(V \otimes W)_{\gamma}}\left(v_{(0)} \otimes w_{(0)}\right) \bar{\gamma}\left(v_{(1)} \otimes w_{(1)}\right) \\
&=\delta^{(V \otimes W)_{\gamma}}\left(\varphi_{V, W}(v \otimes \gamma\right. \\
&
\end{aligned}
$$

where the coaction $\delta^{(V \otimes W)_{\gamma}}$ is given by $\delta^{(V \otimes W)_{\gamma}}: v \otimes w \longmapsto v_{(0)} \otimes w_{(0)} \otimes v_{(1)} w_{(1)}$ (cf. (2.3)). Hence $(\Gamma, \varphi):\left(\mathcal{M}^{H}, \otimes\right) \rightarrow\left(\mathcal{M}^{H_{\gamma}}, \otimes^{\gamma}\right)$ is a monoidal functor.

The monoidal categories are equivalent (actually they are isomorphic) because $\bar{\gamma}$ twists back $H_{\gamma}$ to $H$ and $V_{\gamma}$ to $V$ so that the monoidal functor $(\Gamma, \varphi)$ has an inverse $(\bar{\Gamma}, \bar{\varphi})$, where $\bar{\Gamma}: \mathcal{M}^{H_{\gamma}} \rightarrow \mathcal{M}^{H}$ is the inverse of the functor $\Gamma$ and $\bar{\varphi}_{V_{\gamma}, W_{\gamma}}:\left(V_{\gamma}\right)_{\bar{\gamma}} \otimes\left(W_{\gamma}\right)_{\bar{\gamma}} \rightarrow\left(V_{\gamma} \otimes W_{\gamma}\right)_{\bar{\gamma}}$, $v \otimes w \mapsto v_{(0)} \otimes \gamma w_{(0)} \gamma\left(v_{(1)} \otimes w_{(1)}\right)$. 
If $V \in \mathcal{M}^{H}$ carries additional structures, i.e. if it is an object in $\mathcal{A}^{H},{ }_{A} \mathcal{M}^{H}, \mathcal{M}_{A}{ }^{H}$ or ${ }_{A} \mathcal{M}_{A}{ }^{H}$ (with $A \in \mathcal{A}^{H}$ ), then these additional structures are also deformed by the 2-cocycle $\gamma: H \otimes H \rightarrow \mathbb{K}$. Let us illustrate this for the category $\mathcal{A}^{H}$ of right $H$-comodule algebras: Recall that an object $A \in \mathcal{A}^{H}$ is an object $A \in \mathcal{M}^{H}$ together with two $\mathcal{M}^{H}$-morphisms, $m: A \otimes A \rightarrow A$ and $\eta: \mathbb{K} \rightarrow A$, which satisfy the axioms of an algebra product and unit. Using the functor $\Gamma$ of Theorem 2.19, we can assign to this data the object $\Gamma(A)=A_{\gamma} \in \mathcal{M}^{H_{\gamma}}$ and the two $\mathcal{M}^{H_{\gamma}}$-morphisms $\Gamma(m): \Gamma(A \otimes A) \rightarrow \Gamma(A)$ and $\Gamma(\eta): \Gamma(\mathbb{K}) \rightarrow \Gamma(A)$. The deformed algebra structure $m_{\gamma}, \eta_{\gamma}$ on $A_{\gamma} \in \mathcal{A}^{H_{\gamma}}$ is now defined by using the components $\varphi_{-,-}$(cf. (2.26) ) of the natural isomorphism $\varphi$, and the commutative diagrams
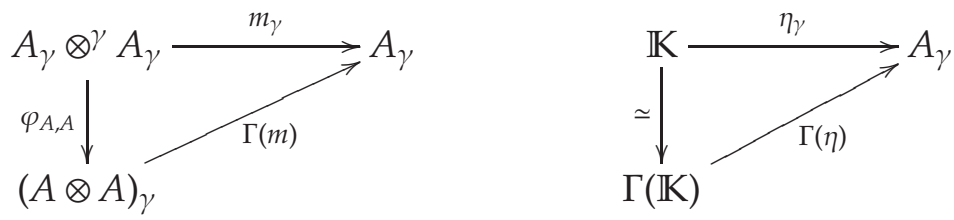

in the category $\mathcal{M}^{H_{\gamma}}$. The deformed product $m_{\gamma}$ is associative due to the 2-cocycle condition of $\gamma$, and $\eta_{\gamma}$ is the unit for $m_{\gamma}$ since $\gamma$ is unital. Explicitly we have that $\eta_{\gamma}=\eta$ and the deformed product reads as

$$
m_{\gamma}: A_{\gamma} \otimes{ }^{\gamma} A_{\gamma} \longrightarrow A_{\gamma}, a \otimes \otimes^{\gamma} a^{\prime} \longmapsto a_{(0)} a_{(0)}^{\prime} \bar{\gamma}\left(a_{(1)} \otimes a_{(1)}^{\prime}\right)=: a \bullet \gamma a^{\prime}
$$

This construction provides us with a functor $\Gamma: \mathcal{A}^{H} \rightarrow \mathcal{A}^{H_{\gamma}}$; indeed it can be easily checked that $\Gamma(\psi):=\psi: A_{\gamma} \rightarrow A_{\gamma}^{\prime}$ is a morphism in $\mathcal{A}^{H_{\gamma}}$ for any $\mathcal{A}^{H}$-morphism $\psi: A \rightarrow A^{\prime}$. Using again the convolution inverse $\bar{\gamma}$ of $\gamma$ we can twist back $A_{\gamma}$ to $A$. In summary we have obtained

Proposition 2.20. Given a 2-cocycle $\gamma: H \otimes H \rightarrow \mathbb{K}$ the functor $\Gamma: \mathcal{A}^{H} \rightarrow \mathcal{A}^{H_{\gamma}}$ induces an equivalence of categories.

By a similar construction one obtains the functors (all denoted by the same symbol) $\Gamma:{ }_{A} \mathcal{M}^{H} \rightarrow{ }_{A_{\gamma}} \mathcal{M}^{H_{\gamma}}, \Gamma: \mathcal{M}_{A}{ }^{H} \rightarrow \mathcal{M}_{A_{\gamma}}{ }^{H_{\gamma}}$ and $\Gamma:{ }_{A} \mathcal{M}_{A}{ }^{H} \rightarrow{ }_{A_{\gamma}} \mathcal{M}_{A_{\gamma}}{ }^{H_{\gamma}}$. Explicitly, the deformed left $A_{\gamma}$-actions are given by

$$
\begin{aligned}
\triangleright_{V_{\gamma}}: A_{\gamma} \otimes^{\gamma} V_{\gamma} & \longrightarrow V_{\gamma}, \\
a \otimes^{\gamma} v & \longmapsto\left(a_{(0)} \triangleright_{V} v_{(0)}\right) \bar{\gamma}\left(a_{(1)} \otimes v_{(1)}\right),
\end{aligned}
$$

while the deformed right $A_{\gamma}$-actions read as

$$
\begin{aligned}
\triangleleft_{V_{\gamma}}: V_{\gamma} \otimes{ }^{\gamma} A_{\gamma} & \longrightarrow V_{\gamma}, \\
v \otimes \otimes^{\gamma} a & \longmapsto\left(v_{(0)} \triangleleft_{V} a_{(0)}\right) \bar{\gamma}\left(v_{(1)} \otimes a_{(1)}\right) .
\end{aligned}
$$

The $A_{\gamma}$-module and $A_{\gamma}$-bimodule properties follow again from the 2-cocycle condition and unitality of $\gamma$. Moreover the bifunctors $\otimes$ described in the Remarks 2.5, 2.7 and 2.8 on module categories are preserved, i.e., the $\mathcal{M}^{H_{\gamma}}$-isomorphisms (2.26), that in the context of Theorem 2.19 define the natural isomorphism $\varphi: \otimes^{\gamma} \circ(\Gamma \times \Gamma) \Rightarrow \Gamma \circ \otimes$, now are respectively $A_{\gamma} \mathcal{M}^{H_{\gamma}}$, $\mathcal{M}_{A_{\gamma}}{ }^{H_{\gamma}}$ and ${ }_{A_{\gamma}} \mathcal{M}_{A_{\gamma}}{ }^{H_{\gamma}}$-isomorphisms; they define the natural isomorphism $\varphi: \otimes{ }^{\gamma} \circ(\Gamma \times \Gamma) \Rightarrow$ $\Gamma \circ \otimes$, where here $\Gamma$ is any of the three functors described above, and where $\otimes \gamma^{\prime}$ is the tensor product corresponding to the Hopf algebra $\mathrm{H}_{\gamma}$. 
For example we here prove that, for all $V \in{ }_{A} \mathcal{M}^{H}$ and $W \in \mathcal{M}^{H}$, the $\mathcal{M}^{H}$-isomorphism $\varphi_{V, W}: V_{\gamma} \otimes W_{\gamma} \rightarrow(V \otimes W)_{\gamma}$ given in (2.26) is an ${ }_{A_{\gamma}} \mathcal{M}^{H_{\gamma}}$-isomorphism. On the one hand, from definition (2.29) we have

$$
\begin{aligned}
\varphi_{V, W}\left(a \triangleright_{V_{\gamma} \otimes \gamma} W_{\gamma}\left(v \otimes^{\gamma} w\right)\right) & \left.=\varphi_{V, W}\left(\left(a \triangleright_{V_{\gamma}} v\right) \otimes^{\gamma} w\right)\right) \\
& =\varphi_{V, W}\left(\left(a_{(0)} \triangleright_{V} v_{(0)}\right) \otimes{ }^{\gamma} w\right) \bar{\gamma}\left(a_{(1)} \otimes v_{(1)}\right) \\
& =\left(a_{(0)} \triangleright_{V} v_{(0)}\right) \otimes w_{(0)} \bar{\gamma}\left(a_{(1)} v_{(1)} \otimes w_{(1)}\right) \bar{\gamma}\left(a_{(2)} \otimes v_{(2)}\right)
\end{aligned}
$$

where in the third line we used (2.7). On the other hand, we have

$$
\begin{aligned}
a \triangleright(V \otimes W)_{\gamma}\left(\varphi_{V, W}(v \otimes \gamma w)\right) & =a \triangleright(V \otimes W)_{\gamma}\left(v_{(0)} \otimes w_{(0)}\right) \bar{\gamma}\left(v_{(1)} \otimes w_{(1)}\right) \\
& =\left(a_{(0)} \triangleright_{V} v_{(0)}\right) \otimes w_{(0)} \bar{\gamma}\left(a_{(1)} \otimes v_{(1)} w_{(1)}\right) \bar{\gamma}\left(v_{(2)} \otimes w_{(2)}\right) .
\end{aligned}
$$

These two expressions coincide because of the 2-cocycle condition (cf. (iii) in Lemma 2.16).

In summary, we have obtained

Proposition 2.21. Given a 2-cocycle $\gamma: H \otimes H \rightarrow \mathbb{K}$ the following functors induce equivalences of categories:

(i) $\Gamma:{ }_{A} \mathcal{M}^{H} \rightarrow{ }_{A_{\gamma}} \mathcal{M}^{H_{\gamma}}$; the left $A_{\gamma}$-actions are defined by (2.29).

(ii) $\Gamma: \mathcal{M}_{A}{ }^{H} \rightarrow \mathcal{M}_{A_{\gamma}}{ }^{H_{\gamma}}$; the right $A_{\gamma}$-actions are defined by (2.30).

(iii) $\Gamma:{ }_{A} \mathcal{M}_{A}{ }^{H} \rightarrow{ }_{A_{\gamma}} \mathcal{M}_{A_{\gamma}}{ }^{H_{\gamma}}$; the $A_{\gamma}$-bimodule structures are defined by (2.29) and (2.30).

In all three cases we have that the maps in (2.26) are isomorphisms in the corresponding categories $\left({ }_{A_{\gamma}} \mathcal{M}^{H_{\gamma}}, \mathcal{M}_{A_{\gamma}}{ }^{H_{\gamma}}\right.$ and ${ }_{A_{\gamma}} \mathcal{M}_{A_{\gamma}}{ }^{H_{\gamma}}$ respectively); they are the components of the natural isomorphism $\varphi: \otimes \gamma \circ(\Gamma \times \Gamma) \Rightarrow \Gamma \circ \otimes$, where the bifunctors $\otimes$ are defined in the Remarks 2.5, 2.7 and 2.8 .

In particular (i) and (ii) induce the following equivalences of $\mathcal{M}^{H}$ - and $\mathcal{M}^{H_{\gamma}}$-module categories: $\left({ }_{A} \mathcal{M}^{H}, \otimes\right) \simeq\left({ }_{A_{\gamma}} \mathcal{M}^{H_{\gamma}, \otimes{ }^{\gamma}}\right)$ and $\left(\mathcal{M}_{A}{ }^{H}, \otimes\right) \simeq\left(\mathcal{M}_{A_{\gamma}}{ }^{H_{\gamma}}, \otimes{ }^{\gamma}\right)$.

We finish this subsection by studying the twisting of the category $\mathrm{C}^{\mathrm{H}}$ of right $\mathrm{H}$-comodule coalgebras. An object $C \in C^{H}$ is an object $C \in \mathcal{M}^{H}$ together with two $\mathcal{M}^{H}$-morphisms $\Delta_{C}: C \rightarrow C \otimes C$ (coproduct) and $\varepsilon_{C}: C \rightarrow \mathbb{K}$ (counit), i.e.

$$
\delta^{C \otimes C} \circ \Delta_{C}=\left(\Delta_{C} \otimes \mathrm{id}\right) \circ \delta^{C}, \quad \delta^{\mathbb{K}} \circ \varepsilon_{C}=\left(\varepsilon_{C} \otimes \mathrm{id}\right) \circ \delta^{C},
$$

which satisfy the axioms of a coalgebra. Morphisms in $C^{H}$ are $H$-comodule maps which are also coalgebra maps (i.e., preserve coproducts and counits). Given now a 2-cocycle $\gamma: H \otimes H \rightarrow \mathbb{K}$, we can use the functor $\Gamma: \mathcal{M}^{H} \rightarrow \mathcal{M}^{H_{\gamma}}$ in order to assign to an object $C \in C^{H}$ (with coproduct $\Delta_{C}$ and counit $\varepsilon_{C}$ ) the object $C_{\gamma} \in C^{H_{\gamma}}$ with coproduct $\Delta_{C \gamma}$ and counit $\varepsilon_{C}$ defined by the commutative diagrams
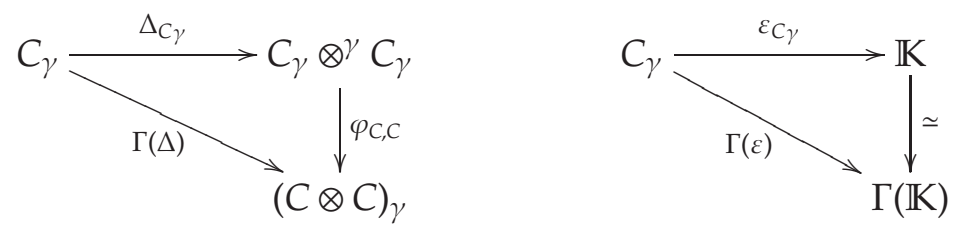
in the category $\mathcal{M}^{H_{\gamma}}$. Notice that $\varepsilon_{C \gamma}=\varepsilon_{C}$ and that the deformed coproduct explicitly reads as

$$
\Delta_{C \gamma}: C_{\gamma} \longrightarrow C_{\gamma} \otimes^{\gamma} C_{\gamma}, C \longmapsto\left(c_{(1)}\right)_{(0)} \otimes^{\gamma}\left(c_{(2)}\right)_{(0)} \gamma\left(\left(c_{(1)}\right)_{(1)} \otimes\left(c_{(2)}\right)_{(1)}\right) .
$$

It is easy to check that $C_{\gamma}$ is an object in $C^{H_{\gamma}}$ and that the assignment $\Gamma: C^{H} \rightarrow C^{H_{\gamma}}$ is a functor (as before, $\Gamma$ acts as the identity on morphisms). In summary, we have obtained

Proposition 2.22. Given a 2-cocycle $\gamma: H \otimes H \rightarrow \mathbb{K}$ the functor $\Gamma: C^{H} \rightarrow C^{H_{\gamma}}$ induces an equivalence of categories.

Example 2.23. The right $H$-comodule $\underline{H}$ is a comodule coalgebra with coproduct and counit canonically inherited from the Hopf algebra $H$, i.e., $\Delta_{\underline{H}}=\Delta$ and $\varepsilon_{\underline{H}}=\varepsilon$. For ease of notation we will omit the indices and denote by $\delta \underline{H}, \Delta, \varepsilon$ the comodule coalgebra structure of $\underline{H}$. Cocycle deformations of $\underline{H}$ will be relevant in $\$ 3.1$.

\subsubsection{Twisting of left $K$-comodules}

Of course, similar twist deformation constructions as in $\$ 2.2 .2$ are available for left Hopf algebra comodules rather than right ones. We briefly collect the corresponding formulae as they will be needed in $\$ 3$. As we later consider also the case where two (in general different) Hopf algebras coact from respectively the left and the right, we denote the Hopf algebra which coacts from the left by $K$.

Let $K$ be a Hopf algebra. A left $K$-comodule is a $\mathbb{K}$-module $V$ together with a $\mathbb{K}$-linear map $\rho^{V}: V \rightarrow K \otimes V$ (called a left $K$-coaction) which satisfies

$$
(\Delta \otimes \mathrm{id}) \circ \rho^{V}=\left(\mathrm{id} \otimes \rho^{V}\right) \circ \rho^{V}, \quad(\varepsilon \otimes \mathrm{id}) \circ \rho^{V}=\mathrm{id} .
$$

The Sweedler notation for the left $K$-coaction is $\rho^{V}(v)=v_{(-1)} \otimes v_{(0)}$ (sum understood), with the $K$-comodule properties (2.36) reading as, for all $v \in V$

$$
\begin{aligned}
\left(v_{(-1)}\right)_{(1)} \otimes\left(v_{(-1)}\right)_{(2)} \otimes v_{(0)} & =v_{(-1)} \otimes\left(v_{(0)}\right)_{(-1)} \otimes\left(v_{(0)}\right)_{(0)}=: v_{(-2)} \otimes v_{(-1)} \otimes v_{(0)}, \\
\varepsilon\left(v_{(-1)}\right) v_{(0)} & =v .
\end{aligned}
$$

We denote by ${ }^{K} \mathcal{M}$ the category of left $K$-comodules; the morphisms in ${ }^{K} \mathcal{M}$ are $\mathbb{K}$-linear maps that preserve the left $K$-coactions, i.e. a $\mathbb{K}$-linear map $\psi: V \rightarrow W$ is a morphism in ${ }^{K} \mathcal{M}$ provided that

$$
\rho^{W} \circ \psi=(\mathrm{id} \otimes \psi) \circ \rho^{V} .
$$

Notice that ${ }^{K} \mathcal{M}$ is a monoidal category with bifunctor $\otimes:{ }^{K} \mathcal{M} \times{ }^{K} \mathcal{M} \rightarrow{ }^{K} \mathcal{M}$ defined by equipping the tensor product (of $\mathbb{K}$-modules) $V \otimes W$ with the tensor product coaction

$$
\begin{aligned}
\rho^{V \otimes W}: V \otimes W & \longrightarrow K \otimes V \otimes W, \\
v \otimes w & \longmapsto v_{(-1)} w_{(-1)} \otimes v_{(0)} \otimes w_{(0)} .
\end{aligned}
$$

The tensor product of morphisms is again $f \otimes g: V \otimes W \rightarrow V^{\prime} \otimes W^{\prime}, v \otimes w \mapsto f(v) \otimes g(w)$ and the unit object in ${ }^{K} \mathcal{M}$ is $\mathbb{K}$ together with the left $K$-coaction $\rho^{\mathbb{K}}:=\eta_{K}: \mathbb{K} \rightarrow K \otimes \mathbb{K} \simeq K$ given by the unit in $K$. 
Let $\sigma: K \otimes K \rightarrow \mathbb{K}$ be a 2-cocycle on $K$ (we use the symbol $\sigma$ in order to distinguish it from 2-cocycles on the Hopf algebra $H$ ). Proposition 2.17 provides us with a deformed Hopf algebra $K_{\sigma}$. We can further construct a functor $\Sigma:{ }^{K} \mathcal{M} \rightarrow{ }^{K}{ }_{\sigma} \mathcal{M}$ by assigning to an object $V \in{ }^{K} \mathcal{M}$ the object $\Sigma(V):={ }_{\sigma} V \in{ }^{K}{ }_{\sigma} \mathcal{M}$, where ${ }_{\sigma} V$ has the same underlying $\mathbb{K}$-module structure of $V$ and left $K_{\sigma}$-coaction $\rho^{\sigma} V:{ }_{\sigma} V \rightarrow K_{\sigma} \otimes{ }_{\sigma} V$ that, as a $\mathbb{K}$-linear map, is given by the left $K$-coaction $\rho^{V}: V \rightarrow K \otimes V$. On morphisms $\psi: V \rightarrow W$ we have $\Sigma(\psi):=\psi:{ }_{\sigma} V \rightarrow{ }_{\sigma} W$. Analogously to Theorem 2.19 we have

Theorem 2.24. The functor $\Sigma:{ }^{K} \mathcal{M} \rightarrow{ }^{K}{ }_{\sigma} \mathcal{M}$ induces an equivalence between the monoidal categories $\left({ }^{K} \mathcal{M}, \otimes\right)$ and $\left({ }^{K_{\sigma}} \mathcal{M},{ }^{\sigma} \otimes\right)$. The natural isomorphism $\varphi^{\ell}:{ }^{\sigma} \otimes \circ(\Gamma \times \Gamma) \Rightarrow \Gamma \circ \otimes$ is given by the ${ }^{K_{\sigma}} \mathcal{M}$ isomorphisms

$$
\begin{aligned}
\varphi_{V, W}^{\ell}:{ }_{\sigma} V^{\sigma} \otimes{ }_{\sigma} W & \longrightarrow \sigma(V \otimes W), \\
v^{\sigma} \otimes w & \longmapsto \sigma\left(v_{(-1)} \otimes w_{(-1)}\right) v_{(0)} \otimes w_{(0)},
\end{aligned}
$$

for all objects $V, W \in{ }^{K} \mathcal{M}$.

The category ${ }^{K} \mathcal{A}$ of left $K$-comodule algebras and the categories ${ }^{K}{ }_{A} \mathcal{M},{ }^{K} \mathcal{M}_{A}$ and ${ }^{K}{ }_{A} \mathcal{M}_{A}$ of relative Hopf modules are defined analogously to the case where the Hopf algebra coacts from the right. As in Remark 2.5 and in Remark $2.7{ }^{K}{ }_{A} \mathcal{M}$ and ${ }^{K} \mathcal{M}_{A}$ are respectively right and left module categories over the monoidal category ${ }^{K} \mathcal{M}$. As in Proposition 2.20 and Proposition 2.21 we obtain

Proposition 2.25. Let $\sigma: K \otimes K \rightarrow \mathbb{K}$ be a 2-cocycle on $K$. Then the monoidal functor $\left(\Sigma, \varphi^{\ell}\right)$ : $\left({ }^{K} \mathcal{M}, \otimes\right) \rightarrow\left({ }^{K_{\sigma}} \mathcal{M},{ }^{\sigma} \otimes\right)$ leads to the following functors, which induce equivalences of categories:

(i) $\Sigma:{ }^{K} \mathcal{A} \rightarrow{ }^{K} \mathcal{A}$; the deformed products are defined by

$$
\begin{aligned}
{ }_{\sigma} m:{ }_{\sigma} A^{\sigma} \otimes_{\sigma} A & \longrightarrow{ }_{\sigma} A, \\
a^{\sigma} \otimes a^{\prime} & \longmapsto \sigma\left(a_{(-1)} \otimes a_{(-1)}^{\prime}\right) a_{(0)} a_{(0)}^{\prime}=: a_{\sigma} \bullet a^{\prime} .
\end{aligned}
$$

(ii) $\Sigma:{ }_{A} \mathcal{M} \rightarrow{ }^{K_{\sigma}}{ }_{\sigma} A \mathcal{M}$; the left ${ }_{\sigma} A$-actions are defined by

$$
\begin{aligned}
\triangleright_{\sigma} V:{ }_{\sigma} A^{\sigma} \otimes{ }_{\sigma} V & \longrightarrow{ }_{\sigma} V, \\
a^{\sigma} \otimes v & \longmapsto \sigma\left(a_{(-1)} \otimes v_{(-1)}\right) a_{(0)} \triangleright_{V} v_{(0)} .
\end{aligned}
$$

(iii) $\Sigma:{ }^{K} \mathcal{M}_{A} \rightarrow{ }^{K} \mathcal{M}_{\sigma}$; the right ${ }_{\sigma} A$-actions are defined by

$$
\begin{aligned}
\triangleleft_{\sigma} V:{ }_{\sigma} V^{\sigma} \otimes{ }_{\sigma} A & \longrightarrow{ }_{\sigma} V, \\
v^{\sigma} \otimes a & \longmapsto \sigma\left(v_{(-1)} \otimes a_{(-1)}\right) v_{(0)} \triangleleft_{V} a_{(0)} .
\end{aligned}
$$

(iv) $\Sigma:{ }_{A} \mathcal{M}_{A} \rightarrow{ }_{\sigma}{ }_{\sigma} A \mathcal{M}_{\sigma}$; the ${ }_{\sigma} A$-bimodule structures are defined by (2.42) and (2.43).

In the cases (ii), (iii) and (iv) we have that the maps in (2.40) are isomorphisms in the corresponding categories $\left(K_{\sigma}{ }_{\sigma} A \mathcal{M}, K_{\sigma} \mathcal{M}_{\sigma} A\right.$ and $K_{\sigma}{ }_{\sigma} A \mathcal{M}_{\sigma} A$ respectively); they are the components of the natural isomorphism $\varphi^{\ell}:{ }^{\sigma} \otimes \circ(\Sigma \times \Sigma) \Rightarrow \Sigma \circ \otimes$, where the bifunctors $\otimes$ are the left comodule analogues of those in Remarks 2.5, 2.7 and 2.8

In particular (ii) and (iii) induce the following equivalences of ${ }^{K} \mathcal{M}$ - and ${ }^{K_{\sigma}} \mathcal{M}$-module categories: $\left({ }_{A}{ }_{A} \mathcal{M}, \otimes\right) \simeq\left({ }_{\sigma}^{K_{\sigma} A} \mathcal{M},{ }^{\sigma} \otimes\right)$ and $\left({ }^{K} \mathcal{M}_{A}, \otimes\right) \simeq\left({ }^{K_{\sigma}} \mathcal{M}_{\sigma} A,{ }^{\sigma} \otimes\right)$. 


\subsubsection{Twisting of $(K, H)$-bicomodules}

Let $K$ and $H$ be two (in general different) Hopf algebras. As our last scenario we consider the situation where we have $\mathbb{K}$-modules $V$ together with a left $K$-coaction $\rho^{V}: V \rightarrow K \otimes V$ and a right $H$-coaction $\delta^{V}: V \rightarrow V \otimes H$ which are compatible in the sense of $(K, H)$-bicomodules, i.e.,

$$
\left(\rho^{V} \otimes \mathrm{id}\right) \circ \delta^{V}=\left(\mathrm{id} \otimes \delta^{V}\right) \circ \rho^{V} .
$$

Evaluated on an element $v \in V$ this condition reads

$$
\left(v_{(0)}\right)_{(-1)} \otimes\left(v_{(0)}\right)_{(0)} \otimes v_{(1)}=v_{(-1)} \otimes\left(v_{(0)}\right)_{(0)} \otimes\left(v_{(0)}\right)_{(1)}=: v_{(-1)} \otimes v_{(0)} \otimes v_{(1)} .
$$

We denote by ${ }^{K} \mathcal{M}^{H}$ the category of $(K, H)$-bicomodules, where ${ }^{K} \mathcal{M}^{H}$-morphisms are $\mathbb{K}$-linear maps that are both $\mathcal{M}^{H}$-comodule and ${ }^{K} \mathcal{M}$-comodule morphisms. It is a monoidal category; the tensor product of $V, W \in{ }^{K} \mathcal{M}^{H}$ is the object $V \otimes W \in{ }^{K} \mathcal{M}^{H}$ with left $K$-comodule structure $\rho^{V \otimes W}$ given in (2.39) and right $H$-comodule structure $\delta^{V \otimes W}$ given in (2.3). Notice that $\delta^{V \otimes W}$ and $\rho^{V \otimes W}$ are compatible in the sense of (2.44),

$$
\begin{aligned}
\left(\rho^{V \otimes W} \otimes \mathrm{id}\right) \circ \delta^{V \otimes W}(v \otimes w) & =\left(v_{(0)}\right)_{(-1)}\left(w_{(0)}\right)_{(-1)} \otimes\left(v_{(0)}\right)_{(0)} \otimes\left(w_{(0)}\right)_{(0)} \otimes v_{(1)} w_{(1)} \\
& =v_{(-1)} w_{(-1)} \otimes v_{(0)} \otimes w_{(0)} \otimes v_{(1)} w_{(1)} \\
& =v_{(-1)} w_{(-1)} \otimes\left(v_{(0)}\right)_{(0)} \otimes\left(w_{(0)}\right)_{(0)} \otimes\left(v_{(0)}\right)_{(1)}\left(w_{(0)}\right)_{(1)} \\
& =v_{(-1)} w_{(-1)} \otimes \delta^{V \otimes W}\left(v_{(0)} \otimes w_{(0)}\right) \\
& =\left(\mathrm{id} \otimes \delta^{V \otimes W}\right) \circ \rho^{V \otimes W}(v \otimes w)
\end{aligned}
$$

for all $v \in V$ and $w \in W$, where in the second and third passage we have used that both $V$ and $W$ are objects in ${ }^{K} \mathcal{M}^{H}$ and so their coactions satisfy the compatibility condition (2.45).

Given a 2-cocycle $\sigma: K \otimes K \rightarrow \mathbb{K}$ and a 2-cocycle $\gamma: H \otimes H \rightarrow \mathbb{K}$, we have by $\$ 2.2 .3$ and $\$ 2.2 .2$ the monoidal functors $\left(\Sigma, \varphi^{\ell}\right):\left({ }^{K} \mathcal{M}^{H}, \otimes\right) \rightarrow\left({ }^{K_{\sigma}} \mathcal{M}^{H},{ }^{\sigma} \otimes\right)$ and $(\Gamma, \varphi):\left({ }^{K} \mathcal{M}^{H}, \otimes\right) \rightarrow\left({ }^{K} \mathcal{M}^{H_{\gamma}}, \otimes^{\gamma}\right)$. We therefore can construct two monoidal functors

$$
\begin{aligned}
& \left(\Sigma, \varphi^{\ell}\right) \circ(\Gamma, \varphi):\left({ }^{K} \mathcal{M}^{H}, \otimes\right) \longrightarrow\left({ }^{K_{\sigma}} \mathcal{M}^{H_{\gamma}},{ }^{\sigma} \otimes^{\gamma}\right), \\
& (\Gamma, \varphi) \circ\left(\Sigma, \varphi^{\ell}\right):\left({ }^{K} \mathcal{M}^{H}, \otimes\right) \longrightarrow\left({ }^{K_{\sigma}} \mathcal{M}^{H_{\gamma},},{ }^{\sigma} \otimes{ }^{\gamma}\right) \text {. }
\end{aligned}
$$

Proposition 2.26. The two monoidal functors in (2.47) are equal.

Proof. As functors, $\Sigma \circ \Gamma$ is equal to $\Gamma \circ \Sigma$ as both functors act as the identity on objects and on morphisms. Thus, we just have to prove that the diagram

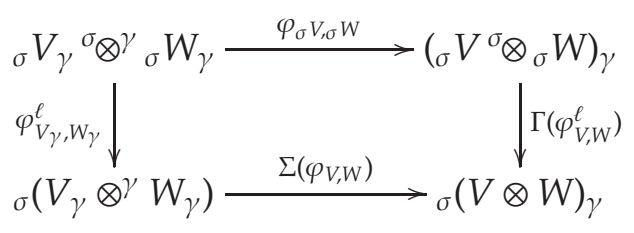


in ${ }^{K_{\sigma}} \mathcal{M}^{H_{\gamma}}$ commutes, for all objects $V, W \in{ }^{K} \mathcal{M}^{H}$; indeed,

$$
\begin{aligned}
& \varphi_{V, W}^{\ell}\left(\varphi_{\sigma} V_{, \sigma} W\left(v^{\sigma} \otimes \gamma w\right)\right)=\varphi_{V, W}^{\ell}\left(v_{(0)}{ }^{\sigma} \otimes w_{(0)}\right) \bar{\gamma}\left(v_{(1)} \otimes w_{(1)}\right) \\
& =\sigma\left(\left(v_{(0)}\right)_{(-1)} \otimes\left(w_{(0)}\right)_{(-1)}\right)\left(v_{(0)}\right)_{(0)} \otimes\left(w_{(0)}\right)_{(0)} \bar{\gamma}\left(v_{(1)} \otimes w_{(1)}\right) \\
& =\sigma\left(v_{(-1)} \otimes w_{(-1)}\right)\left(v_{(0)}\right)_{(0)} \otimes\left(w_{(0)}\right)_{(0)} \bar{\gamma}\left(\left(v_{(0)}\right)_{(1)} \otimes\left(w_{(0)}\right)_{(1)}\right) \\
& =\sigma\left(v_{(-1)} \otimes w_{(-1)}\right) \varphi_{V, W}\left(v_{(0)} \otimes w^{\gamma} w_{(0)}\right) \\
& =\varphi_{V, W}\left(\varphi_{V_{\gamma}, W_{\gamma}}^{\ell}\left(v^{\sigma} \otimes^{\gamma} w\right)\right),
\end{aligned}
$$

for all $v \in V$ and $w \in W$. In the third equality we have used the bicomodule property (2.45) for $V$ and $W$.

In short the above proposition states that it does not matter if we first deform by $\sigma$ and then by $\gamma$ or if we first deform by $\gamma$ and then by $\sigma$.

Let us now consider the category ${ }^{K} \mathcal{A}^{H}$ of $(K, H)$-bicomodule algebras, where objects and morphisms are in ${ }^{K} \mathcal{A}^{H}$ if they are in $\mathcal{A}^{H},{ }^{K} \mathcal{A}$ and ${ }^{K} \mathcal{M}^{H}$. For $A \in{ }^{K} \mathcal{A}^{H}$, we further consider the categories of relative Hopf modules ${ }^{K} \mathcal{M}^{H},{ }^{K} \mathcal{M}_{A}{ }^{H}$ and ${ }^{K}{ }_{A} \mathcal{M}_{A}{ }^{H}$, where by definition objects and morphisms are in ${ }_{A}{ }_{A} \mathcal{M}_{A}{ }^{H}$ if they are in ${ }_{A} \mathcal{M}_{A}{ }^{H},{ }_{A} \mathcal{M}_{A}$ and ${ }^{K} \mathcal{M}^{H}$ (the categories ${ }_{A}{ }_{A} \mathcal{M}^{H}$ and ${ }^{K} \mathcal{M}_{A}{ }^{H}$ are similarly defined). In complete analogy to Propositions 2.20, 2.21 and 2.25 and because of the compatibility between the $K$ - and the $H$-coactions we obtain the following

Proposition 2.27. Let $\sigma: K \otimes K \rightarrow \mathbb{K}$ and $\gamma: H \otimes H \rightarrow \mathbb{K}$ be two 2-cocycles. Then the monoidal functor $(\Gamma, \varphi) \circ\left(\Sigma, \varphi^{\ell}\right)=\left(\Sigma, \varphi^{\ell}\right) \circ(\Gamma, \varphi):{ }^{K} \mathcal{M}^{H} \rightarrow{ }^{K}{ } \mathcal{M}^{H_{\gamma}}$ leads to the following functors, which induce equivalences of categories.

(i) $\Gamma \circ \Sigma=\Sigma \circ \Gamma:{ }^{K} \mathcal{A}^{H} \rightarrow K_{\sigma} \mathcal{A}^{H_{\gamma}}$; the deformed products are defined by

$$
\begin{aligned}
{ }_{\sigma} m_{\gamma}:{ }_{\sigma} A_{\gamma}{ }^{\sigma} \otimes \gamma^{\gamma}{ }_{\sigma} A_{\gamma} & \longrightarrow{ }_{\sigma} A_{\gamma}, \\
a^{\sigma} \otimes{ }^{\gamma} a^{\prime} & \longmapsto \sigma\left(a_{(-1)} \otimes a_{(-1)}\right) a_{(0)} a_{(0)}^{\prime} \bar{\gamma}\left(a_{(1)} \otimes a_{(1)}\right)=: a_{\sigma} \bullet a_{\gamma} a^{\prime} .
\end{aligned}
$$

(ii) $\Gamma \circ \Sigma=\Sigma \circ \Gamma:{ }_{A} \mathcal{M}^{H} \rightarrow{ }_{\sigma}^{K_{\sigma}} A_{\gamma} \mathcal{M}^{H_{\gamma}}$; the left ${ }_{\sigma} A_{\gamma}$-actions are defined by

$$
\begin{aligned}
\triangleright_{\sigma} V_{\gamma}:{ }_{\sigma} A_{\gamma}{ }^{\sigma} \otimes^{\gamma}{ }_{\sigma} V_{\gamma} & \longrightarrow{ }_{\sigma} V_{\gamma}, \\
a^{\sigma} \otimes{ }^{\gamma} v & \longmapsto \sigma\left(a_{(-1)} \otimes v_{(-1)}\right) a_{(0)} \triangleright_{V} v_{(0)} \bar{\gamma}\left(a_{(1)} \otimes v_{(1)}\right) .
\end{aligned}
$$

(iii) $\Gamma \circ \Sigma=\Sigma \circ \Gamma:{ }^{K} \mathcal{M}_{A}{ }^{H} \rightarrow{ }^{K_{\sigma}} \mathcal{M}_{\sigma} A_{\gamma}{ }^{H}$; the right ${ }_{\sigma} A_{\gamma}$-actions are defined by

$$
\begin{aligned}
\triangleleft_{\sigma} V_{\gamma}:{ }_{\sigma} V_{\gamma}{ }^{\sigma} \otimes{ }^{\gamma}{ }_{\sigma} A_{\gamma} & \longrightarrow{ }_{\sigma} V_{\gamma}, \\
v^{\sigma} \otimes{ }^{\gamma} a & \longmapsto \sigma\left(v_{(-1)} \otimes a_{(-1)}\right) v_{(0)} \triangleleft_{V} a_{(0)} \bar{\gamma}\left(v_{(1)} \otimes a_{(1)}\right) .
\end{aligned}
$$

(iv) $\Gamma \circ \Sigma=\Sigma \circ \Gamma:{ }_{A} \mathcal{M}_{A}{ }^{H} \rightarrow{ }_{\sigma}^{K_{\sigma}} A_{\gamma} \mathcal{M}_{\sigma} A_{\gamma}{ }^{H_{\gamma}}$; the ${ }_{\sigma} A_{\gamma}$-bimodule structures are defined by (2.50) and (2.51).

In the cases (ii), (iii) and (iv), $\Gamma\left(\varphi_{V, W}^{\ell}\right) \circ \varphi_{\sigma} V_{, \sigma} W$ are isomorphisms in the corresponding categories $\left(K_{\sigma}{ }_{\sigma} A_{\gamma} \mathcal{M}^{H_{\gamma}}, K_{\sigma} \mathcal{M}_{\sigma} A_{\gamma}{ }^{H_{\gamma}}\right.$ and ${ }_{{ }_{\sigma}} A_{\gamma} \mathcal{M}_{\sigma} A_{\gamma}{ }^{H_{\gamma}}$ respectively); they are the components of the natural isomorphism $\Gamma\left(\varphi^{\ell}\right) \circ \varphi:{ }^{\sigma} \otimes \gamma \circ(\Gamma \circ \Sigma \times \Gamma \circ \Sigma) \Rightarrow \Gamma \circ \Sigma \circ \otimes$, where the bifunctors $\otimes$ are the bicomodule analogues of those in the Remarks 2.5, 2.7 and 2.8 .

In particular (ii) and (iii) induce the following equivalences of ${ }^{\mathrm{K}} \mathcal{M}^{H}$-and ${ }^{K_{\sigma}} \mathcal{M}^{H_{\gamma}}$-module categories: $\left({ }_{A} \mathcal{M}^{H}, \otimes\right) \simeq\left({ }_{\sigma}^{K_{\sigma}} A_{\gamma} \mathcal{M}^{H_{\gamma}},{ }^{\sigma} \otimes{ }^{\gamma}\right)$ and $\left({ }^{K} \mathcal{M}_{A}{ }^{H}, \otimes\right) \simeq\left({ }^{K_{\sigma}} \mathcal{M}_{\sigma} A_{\gamma}{ }^{H_{\gamma}},{ }^{\sigma} \otimes{ }^{\gamma}\right)$. 


\section{Twisting of Hopf-Galois extensions}

Suppose $B=A^{c o H} \subseteq A$ is an $H$-Hopf-Galois extension with total space $A$, base space $B$ and structure Hopf algebra $H$ (see Definition 2.9). We are interested in studying how the invertibility of the canonical map $\chi$ behaves under deformations via 2-cocycles. We are in particular interested in deforming classical principal bundles (cf. Example 2.13) in order to obtain noncommutative principal bundles or quantum principal bundles, i.e., principal comodule algebras (cf. Definition 2.14) obtained via deformation of principal bundles. Let us observe that if we consider a 2-cocycle on the structure Hopf algebra $H$ and use it to twist the data $(A, B, H)$, the deformation of the base space $B$ turns out to be trivial as a direct consequence of the triviality of the right $H$-coaction on coinvariants. In the language of noncommutative principal bundles this means that by twisting a classical principal bundle with a 2-cocycle on $H$, we only have the possibility to obtain a noncommutative principal bundle with a classical (i.e. not deformed) base space. In order to obtain a more general theory, which also allows for deformations of the base space, we shall also consider the case of $A$ carrying an external symmetry (described by a second Hopf algebra $K$ ) that is compatible with the right $H$-comodule structure. Indeed, by assuming the total space $A$ to be a $(K, H)$-bicomodule algebra, we can use a 2-cocycle on $K$ to induce a deformation of $A$, which in general also deforms the subalgebra $B$ of $H$-coinvariants.

Notice that it would be also possible to develop this theory by assuming the existence of an action of a Hopf algebra $\mathcal{U}$ (dual to $K$ ) and use a twist $\mathcal{F} \in \mathcal{U} \otimes \mathcal{U}$, rather than a 2-cocycle on $K$, to implement the deformation (see the discussion in Appendix $\mathrm{A}$ ). Nevertheless, we shall use here the language of coactions as usual in the literature on Hopf-Galois extensions.

Example 3.1. In the setting of Example 2.13, a natural choice for the Hopf algebra $\mathcal{U}$ is the universal enveloping algebra of the Lie algebra of $G$-equivariant vector fields on $P$, i.e. the Lie algebra of derivations of $A=C^{\infty}(P)$ which commute with the right G-action. The Hopf algebra $\mathcal{U}$ describes the infinitesimal automorphisms of the principal G-bundle $\pi: P \rightarrow M$. A natural choice for the Hopf algebra $K$ would be the Hopf algebra of functions on a finitedimensional Lie subgroup of the group of automorphisms $\phi: P \rightarrow P$ of the bundle.

We therefore consider the following three scenarios:

\$3.1 A deformation based on a 2-cocycle on the structure Hopf algebra $H$. Here the total space $A$ and the structure Hopf algebra $H$ are twisted, while the base space $B$ is undeformed.

83.2 A deformation based on a 2-cocycle on an external Hopf algebra $K$ of symmetries of $A$ with $H$-equivariant coaction. Here the total space $A$ and the base $B$ are twisted, while the structure Hopf algebra $H$ is undeformed.

$\$ 3.3$ The combination of the previous two cases. Here the total space $A$, the structure Hopf algebra $H$ and the base space $B$ are all twisted in a compatible way.

In all these cases we shall show that Hopf-Galois extensions and principal comodule algebras are respectively deformed into Hopf-Galois extensions and principal comodule algebras. In particular principal bundles are deformed into noncommutative principal bundles. Our proof relies on relating the canonical map of the twisted bundle with the canonical map of the original bundle via a commutative diagram in the appropriate category. 


\subsection{Deformation via a 2-cocycle on the structure Hopf algebra $H$}

Let $\gamma: H \otimes H \rightarrow \mathbb{K}$ be a 2-cocycle on $H$ which we use to deform $H$ into a new Hopf algebra $H_{\gamma}$ with the same co-structures and unit, but different product and antipode given in Proposition 2.17 Using the techniques from \$2.2.2, we can deform $A \in \mathcal{A}^{H}$ into $A_{\gamma} \in \mathcal{A}^{H_{\gamma}}$ by introducing the twisted product (2.28). As we have already observed above, the algebra structure of the subalgebra of $H$-coinvariants $B \subseteq A$ does not change under our present class of 2-cocycle deformations, since the coaction of $H$ on the elements of $B$ is trivial. In other words, the subalgebra of coinvariants $B_{\gamma}=A_{\gamma}^{\mathrm{coH}_{\gamma}}$ of $A_{\gamma}$ is isomorphic (via the identity map) to $B=A^{\mathrm{coH}}$ as an algebra (see (2.28) ).

We shall relate the twisted canonical map $\chi_{\gamma}: A_{\gamma} \otimes_{B}^{\gamma} A_{\gamma} \rightarrow A_{\gamma} \otimes^{\gamma} H_{\gamma}$ with the original one $\chi: A \otimes_{B} A \rightarrow A \otimes \underline{H}$ by understanding both as morphisms in the category ${ }_{A_{\gamma}} \mathcal{M}_{A_{\gamma}}{ }{ }_{\gamma}$. Our strategy is as follows: First, we notice that applying the functor $\Gamma:{ }_{A} \mathcal{M}_{A}{ }^{H} \rightarrow{ }_{A_{\gamma}} \mathcal{M}_{A_{\gamma}} H_{\gamma}$ from Proposition 2.21 (iii) on the original canonical map $\chi$ (which is a morphism in ${ }_{A} \mathcal{M}_{A}{ }^{H}$, cf. Proposition 2.10), we obtain the morphism $\Gamma(\chi):\left(A \otimes_{B} A\right)_{\gamma} \rightarrow(A \otimes \underline{H})_{\gamma}$ in ${ }_{A_{\gamma}} \mathcal{M}_{A_{\gamma}} H_{\gamma}$. Next, we relate the two morphisms $\Gamma(\chi)$ and $\chi_{\gamma}$ in ${ }_{A_{\gamma}} \mathcal{M}_{A_{\gamma}}{ }^{H_{\gamma}}$ via the natural transformation $\varphi_{-,-}$ (cf. (2.26) and an isomorphism (5 introduced in Theorem 3.4 below after a few technical lemmas. The role of the isomorphism $6 \mathfrak{5}$ is to relate the two twist deformations of $H$ into right $H_{\gamma}$-comodule coalgebras: $\underline{H}_{\gamma}$ and $\underline{H}_{\gamma}$; recall Example 2.23. While $\underline{H}_{\gamma}$ is the deformation of the $H$-comodule coalgebra $\underline{H}$, in $H_{\gamma}$ we first deform the Hopf algebra $H$ to $H_{\gamma}$ and then regard it as an $H_{\gamma}$-comodule coalgebra. The isomorphism $\mathfrak{G}_{\mathfrak{5}}$ is related to the natural isomorphism proving the equivalence of the categories of Hopf algebra modules and twisted Hopf algebra modules as closed categories, cf. Appendix B,

Let us now discuss the construction in detail. By Proposition 2.10 we have that the canonical map $\chi: A \otimes_{B} A \rightarrow A \otimes \underline{H}$ is a morphism in ${ }_{A} \mathcal{M}_{A}{ }^{H}$. Applying the functor $\Gamma$ : ${ }_{A} \mathcal{M}_{A}{ }^{H} \rightarrow{ }_{A_{\gamma}} \mathcal{M}_{A_{\gamma}}{ }^{H_{\gamma}}$ from Proposition 2.21(iii) we obtain the ${ }_{A_{\gamma}} \mathcal{M}_{A_{\gamma}}{ }^{H_{\gamma}}$-morphism $\Gamma(\chi)=\chi$ : $\left(A \otimes_{B} A\right)_{\gamma} \rightarrow(A \otimes \underline{H})_{\gamma^{\prime}}$, where $\left(A \otimes_{B} A\right)_{\gamma^{\prime}}=\Gamma\left(A \otimes_{B} A\right)$ and $(A \otimes \underline{H})_{\gamma}=\Gamma(A \otimes \underline{H})$ are objects in ${ }_{A_{\gamma}} \mathcal{M}_{A_{\gamma}}{ }^{H_{\gamma}}$, while $A \otimes_{B} A$ and $A \otimes \underline{H}$ are in ${ }_{A} \mathcal{M}_{A}{ }^{H}$. Explicitly, the left $A_{\gamma}$-action on $\left(A \otimes_{B} A\right)_{\gamma}$ reads

$$
\begin{aligned}
\triangleright_{\left(A \otimes_{B} A\right)_{\gamma}: A_{\gamma} \otimes^{\gamma}\left(A \otimes_{B} A\right)_{\gamma}} & \longrightarrow\left(A \otimes_{B} A\right)_{\gamma}, \\
c \otimes^{\gamma}\left(a \otimes_{B} a^{\prime}\right) & \longmapsto c_{(0)} a_{(0)} \otimes_{B} a_{(0)}^{\prime} \bar{\gamma}\left(c_{(1)} \otimes a_{(1)} a_{(1)}^{\prime}\right) .
\end{aligned}
$$

The right $H_{\gamma}$-coaction on $\left(A \otimes_{B} A\right)_{\gamma}$ is given by the right $H$-coaction on $A \otimes_{B} A$, and the right $A_{\gamma}$-action reads

$$
\begin{aligned}
\triangleleft_{\left(A \otimes_{B} A\right)_{\gamma}:\left(A \otimes_{B} A\right)_{\gamma} \otimes^{\gamma} A_{\gamma}} & \longrightarrow\left(A \otimes_{B} A\right)_{\gamma}, \\
\left(a \otimes_{B} a^{\prime}\right) \otimes^{\gamma} c & \longmapsto a_{(0)} \otimes_{B} a_{(0)}^{\prime} C_{(0)} \bar{\gamma}\left(a_{(1)} a^{\prime}{ }_{(1)} \otimes \mathcal{C}_{(1)}\right) .
\end{aligned}
$$

Analogously, on $(A \otimes \underline{H})_{\gamma}$ the left $A_{\gamma}$-action reads

$$
\begin{aligned}
\nabla_{(A \otimes \underline{H})_{\gamma}}: A_{\gamma} \otimes^{\gamma}(A \otimes \underline{H})_{\gamma} & \longrightarrow(A \otimes \underline{H})_{\gamma}, \\
c \otimes \otimes^{\gamma}(a \otimes h) & \longmapsto c_{(0)} a_{(0)} \otimes h_{(2)} \bar{\gamma}\left(c_{(1)} \otimes a_{(1)} S\left(h_{(1)}\right) h_{(3)}\right),
\end{aligned}
$$

the right $H_{\gamma}$-coaction on $(A \otimes \underline{H})_{\gamma}$ is given by the right $H$-coaction on $A \otimes \underline{H}$, and the right 
$A_{\gamma}$-action reads

$$
\begin{aligned}
\triangleleft(A \otimes \underline{H})_{\gamma}:(A \otimes \underline{H})_{\gamma} \otimes \gamma^{\gamma} A_{\gamma} & \longrightarrow(A \otimes \underline{H})_{\gamma}, \\
(a \otimes h) \otimes^{\gamma} c & \longmapsto a_{(0)} c_{(0)} \otimes h_{(2)} c_{(1)} \bar{\gamma}\left(a_{(1)} S\left(h_{(1)}\right) h_{(3)} \otimes c_{(2)}\right) .
\end{aligned}
$$

We proceed with the second step and introduce the isomorphism 65 mentioned above relating the two deformations of $H$ when thought of as a Hopf algebra or as a right $H$ comodule coalgebra $\underline{H}$. We first need the following technical lemmas. Recall the definition of $u_{\gamma}$ and its convolution inverse from (2.22).

Lemma 3.2. Every 2-cocycle $\gamma: H \otimes H \rightarrow \mathbb{K}$ satisfies

$$
\begin{gathered}
u_{\gamma}\left(h_{(1)}\right) \bar{\gamma}\left(S\left(h_{(2)}\right) \otimes k\right)=\gamma\left(h_{(1)} \otimes S\left(h_{(2)}\right) k\right), \\
\bar{u}_{\gamma}\left(h_{(1)}\right) \gamma\left(h_{(2)} \otimes k\right)=\bar{\gamma}\left(S\left(h_{(1)}\right) \otimes h_{(2)} k\right), \\
\gamma\left(g h_{(1)} \otimes S\left(h_{(2)}\right) k\right)=\bar{\gamma}\left(g_{(1)} \otimes h_{(1)}\right) u_{\gamma}\left(h_{(2)}\right) \bar{\gamma}\left(S\left(h_{(3)}\right) \otimes k_{(1)}\right) \gamma\left(g_{(2)} \otimes k_{(2)}\right),
\end{gathered}
$$

for all $g, h, k \in H$.

Proof. Recalling the definition of $u_{\gamma}$ and Lemma 2.16 (iv) we have (3.5). Similarly from Lemma 2.16 (iii) we have (3.6). From Lemma 2.16 (iii) we also obtain (recall $\bar{\gamma} * \gamma=\varepsilon \otimes \varepsilon$ )

$$
\gamma(g h \otimes k)=\bar{\gamma}\left(g_{(1)} \otimes h_{(1)}\right) \gamma\left(h_{(2)} \otimes k_{(1)}\right) \gamma\left(g_{(2)} \otimes h_{(3)} k_{(2)}\right),
$$

for all $g, h, k \in H$. Use of this identity and of (3.5) proves (3.7):

$$
\begin{aligned}
\gamma\left(g h_{(1)} \otimes S\left(h_{(2)}\right) k\right) & =\bar{\gamma}\left(g_{(1)} \otimes h_{(1)}\right) \gamma\left(h_{(2)} \otimes S\left(h_{(5)}\right) k_{(1)}\right) \gamma\left(g_{(2)} \otimes h_{(3)} S\left(h_{(4)}\right) k_{(2)}\right) \\
& =\bar{\gamma}\left(g_{(1)} \otimes h_{(1)}\right) \gamma\left(h_{(2)} \otimes S\left(h_{(3)}\right) k_{(1)}\right) \gamma\left(g_{(2)} \otimes k_{(2)}\right) \\
& =\bar{\gamma}\left(g_{(1)} \otimes h_{(1)}\right) u_{\gamma}\left(h_{(2)}\right) \bar{\gamma}\left(S\left(h_{(3)}\right) \otimes k_{(1)}\right) \gamma\left(g_{(2)} \otimes k_{(2)}\right) .
\end{aligned}
$$

Lemma 3.3. Let $\underline{H}$ be the right $H$-comodule coalgebra with adjoint coaction $\delta \underline{H}=$ Ad : $\underline{H} \rightarrow$ $\underline{H} \otimes H, h \mapsto h_{(2)} \otimes S\left(h_{(1)}\right) h_{(3)}$. Then the twisted right $H_{\gamma}$-comodule coalgebra $\underline{H}_{\gamma}$, has coproduct

$$
\Delta_{\gamma}(h)=h_{(3)} \otimes^{\gamma} h_{(7)} \bar{\gamma}\left(S\left(h_{(2)}\right) \otimes h_{(4)}\right) u_{\gamma}\left(h_{(5)}\right) \bar{\gamma}\left(S\left(h_{(6)}\right) \otimes h_{(8)}\right) \gamma\left(S\left(h_{(1)}\right) \otimes h_{(9)}\right) .
$$

Proof. By the general theory, the twisted right $H_{\gamma}$-comodule coalgebra $\underline{H}_{\gamma}$ has coproduct given by $\Delta_{\gamma}(h)=h_{(2)} \otimes^{\gamma} h_{(5)} \gamma\left(S\left(h_{(1)}\right) h_{(3)} \otimes S\left(h_{(4)}\right) h_{(6)}\right)$ (see (2.35)), then (3.8) follows by applying (3.7) above.

On the other hand, the twisted Hopf algebra $H_{\gamma}$ can be considered as a right $H_{\gamma}$-comodule coalgebra, denoted by $H_{\gamma}$, via the $H_{\gamma}$-adjoint coaction

$$
\delta \underline{H_{\gamma}}=\operatorname{Ad}_{\gamma}: \underline{H_{\gamma}} \longrightarrow \underline{H_{\gamma}} \otimes H_{\gamma}, h \longmapsto h_{(2)} \otimes S_{\gamma}\left(h_{(1)}\right) \cdot \gamma h_{(3)}
$$

and the coproduct $\Delta: \underline{H_{\gamma}} \rightarrow \underline{H_{\gamma}} \otimes H^{\gamma}, h \mapsto h_{(1)} \otimes \gamma h_{(2)}$. 
Theorem 3.4. The K-linear map

$$
\text { (5 }: \underline{H_{\gamma}} \longrightarrow \underline{H}_{\gamma}, h \longmapsto h_{(3)} u_{\gamma}\left(h_{(1)}\right) \bar{\gamma}\left(S\left(h_{(2)}\right) \otimes h_{(4)}\right)
$$

is an isomorphism of right $H_{\gamma}$-comodule coalgebras, with inverse

$$
\mathfrak{G}^{-1}: \underline{H}_{\gamma} \longrightarrow \underline{H}_{\gamma}, h \longmapsto h_{(3)} \bar{u}_{\gamma}\left(h_{(2)}\right) \gamma\left(S\left(h_{(1)}\right) \otimes h_{(4)}\right) .
$$

Proof. It is easy to prove by a direct calculation that $\mathfrak{5}^{-1}$ is the inverse of 65 . We now show that $\mathfrak{G}$ is a right $H_{\gamma}$-comodule morphism, for all $h \in \underline{H_{\gamma}}$,

$$
\begin{aligned}
(\mathfrak{5} \otimes & \mathrm{id})\left(\operatorname{Ad}_{\gamma}(h)\right)= \\
& =\left(\mathfrak{G}\left(h_{(2)}\right) \otimes S_{\gamma}\left(h_{(1)}\right) \cdot \gamma h_{(3)}\right. \\
& =\left(\mathfrak{5}\left(h_{(4)}\right) \otimes u_{\gamma}\left(h_{(1)}\right) S\left(h_{(2)}\right) \bar{u}_{\gamma}\left(h_{(3)}\right) \cdot \gamma h_{(5)}\right. \\
& =u_{\gamma}\left(h_{(6)}\right) h_{(8)} \bar{\gamma}\left(S\left(h_{(7)}\right) \otimes h_{(9)}\right) \otimes u_{\gamma}\left(h_{(1)}\right) \bar{u}_{\gamma}\left(h_{(5)}\right) \gamma\left(S\left(h_{(4)}\right) \otimes h_{(10)}\right) S\left(h_{(3)}\right) h_{(11)} \bar{\gamma}\left(S\left(h_{(2)}\right) \otimes h_{(12)}\right) \\
& =h_{(6)} \bar{\gamma}\left(S\left(h_{(5)}\right) \otimes h_{(7)}\right) \otimes u_{\gamma}\left(h_{(1)}\right) \gamma\left(S\left(h_{(4)}\right) \otimes h_{(8)}\right) S\left(h_{(3)}\right) h_{(9)} \bar{\gamma}\left(S\left(h_{(2)}\right) \otimes h_{(10)}\right) \\
& \left.=u_{\gamma}\left(h_{(1)}\right) h_{(4)} \otimes S\left(h_{(3)}\right) h_{(5)}\right) \bar{\gamma}\left(S\left(h_{(2)}\right) \otimes h_{(6)}\right)=\operatorname{Ad}((\mathfrak{5}(h)),
\end{aligned}
$$

where in the fourth passage we used $u_{\gamma}\left(h_{(6)}\right) \bar{u}_{\gamma}\left(h_{(5)}\right)=\varepsilon\left(h_{(5)}\right)$, and in the fifth $h_{(6)} \bar{\gamma}\left(S\left(h_{(5)}\right) \otimes\right.$ $\left.h_{(7)}\right) \gamma\left(S\left(h_{(4)}\right) \otimes h_{(8)}\right)=h_{(5)} \varepsilon\left(h_{(4)}\right) \varepsilon\left(h_{(6)}\right)$. Next, we prove that $(5$ is a coalgebra morphism, i.e. $\left(\mathfrak{5} \otimes \gamma(\mathfrak{5}) \circ \Delta=\Delta_{\gamma} \circ(\mathfrak{5}\right.$,

$$
\begin{aligned}
\left(\mathfrak{5} \otimes^{\gamma}(\mathfrak{5}) \circ \Delta \circ \mathfrak{5}^{-1}(h)\right. & =\bar{u}_{\gamma}\left(h_{(2)}\right) \mathfrak{F}_{\left(h_{(3)}\right) \otimes \gamma}\left(\mathfrak{5}\left(h_{(4)}\right) \gamma\left(S\left(h_{(1)}\right) \otimes h_{(5)}\right)\right. \\
& =\bar{u}_{\gamma}\left(h_{(2)}\right) u_{\gamma}\left(h_{(3)}\right) \bar{\gamma}\left(S\left(h_{(4)}\right) \otimes h_{(6)}\right) h_{(5)} \otimes^{\gamma}\left(\mathfrak{5}\left(h_{(7)}\right) \gamma\left(S\left(h_{(1)}\right) \otimes h_{(8)}\right)\right. \\
& =\bar{\gamma}\left(S\left(h_{(2)}\right) \otimes h_{(4)}\right) h_{(3)} \otimes^{\gamma}\left(\mathfrak{5}\left(h_{(5)}\right) \gamma\left(S\left(h_{(1)}\right) \otimes h_{(6)}\right)\right. \\
& =\bar{\gamma}\left(S\left(h_{(2)}\right) \otimes h_{(4)}\right) h_{(3)} \otimes^{\gamma} h_{(7)} u_{\gamma}\left(h_{(5)}\right) \bar{\gamma}\left(S\left(h_{(6)}\right) \otimes h_{(8)}\right) \gamma\left(S\left(h_{(1)}\right) \otimes h_{(9)}\right) \\
& =\Delta_{\gamma}(h),
\end{aligned}
$$

for all $h \in \underline{H}_{\gamma}$. The last equality follows from comparison with 3.8).

Remark 3.5. If we dualize this picture by considering a dually paired Hopf algebra $H^{\prime}$ (and dual modules) then the right $H$-adjoint coaction dualizes into the right $H^{\prime}$-adjoint action, $\zeta \triangleleft \xi=S\left(\xi_{(1)}\right) \zeta \xi_{(2)}$ for all $\zeta, \xi \in H^{\prime}$. If we further consider a mirror construction by using left adjoint actions rather than right ones, then the analogue of the isomorphism 65 is the isomorphism $D$ studied in [2] and more in general in [4]. Explicitly, as explained in Appendix B. the isomorphism 65 is dual to the isomorphism $D$ relative to the Hopf algebra $H^{\prime o p}$ cop with opposite product and coproduct; this latter is a component of a natural transformation determining the equivalence of the closed monoidal categories of left $H^{\prime o p}{ }^{c o p}$-modules and left $\left(H_{\gamma}^{\prime}\right)^{o p ~ c o p}$-modules.

After these preliminaries we can now relate the twisted canonical map $\chi_{\gamma}$ with the original one $\chi$.

Theorem 3.6. Let $H$ be a Hopf algebra and $A$ an $H$-comodule algebra. Consider the algebra extension $B=A^{c o H} \subseteq A$ and the associated canonical map $\chi: A \otimes_{B} A \longrightarrow A \otimes H$. Given a 2-cocycle 
$\gamma: H \otimes H \rightarrow \mathbb{K}$ the diagram

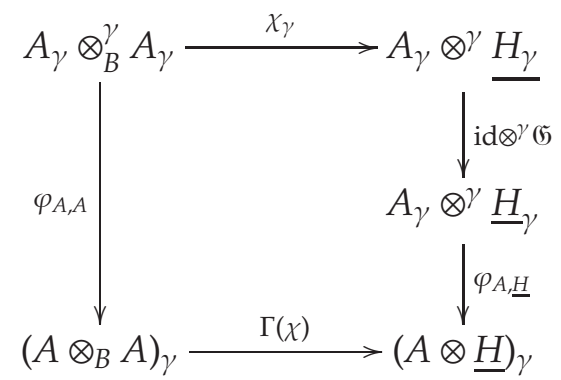

in ${ }_{A_{\gamma}} \mathcal{M}_{A_{\gamma}}{ }^{H_{\gamma}}$ commutes.

Proof. A straightforward computation shows that $\varphi_{A, A}: A_{\gamma} \otimes \gamma A_{\gamma} \rightarrow(A \otimes A)_{\gamma}$ descends to a well-defined isomorphism on the quotients $\varphi_{A, A}: A_{\gamma} \otimes_{B}^{\gamma} A_{\gamma} \rightarrow\left(A \otimes_{B} A\right)_{\gamma}$, i.e. the left vertical arrow is well defined. We prove that the diagram (3.12) commutes. We obtain for the composition (id $\otimes^{\gamma}(\mathfrak{5}) \circ \chi_{\gamma}$ the following expression

$$
\begin{aligned}
\left(\operatorname{id} \otimes^{\gamma}(\mathfrak{5})\left(\chi_{\gamma}\left(a \otimes_{B}^{\gamma} a^{\prime}\right)\right)\right. & =a_{(0)} a^{\prime}{ }_{(0)} \otimes^{\gamma}\left(\mathfrak{F}\left(a^{\prime}{ }_{(2)}\right) \bar{\gamma}\left(a_{(1)} \otimes a^{\prime}{ }_{(1)}\right)\right. \\
& =a_{(0)} a^{\prime}{ }_{(0)} \otimes^{\gamma} a^{\prime}{ }_{(4)} u_{\gamma}\left(a^{\prime}{ }_{(2)}\right) \bar{\gamma}\left(S\left(a_{(3)}^{\prime}\right) \otimes a_{(5)}^{\prime}\right) \bar{\gamma}\left(a_{(1)} \otimes a^{\prime}{ }_{(1)}\right) .
\end{aligned}
$$

On the other hand, from (2.26) and (2.14) we have

$$
\varphi_{A, \underline{H}}^{-1}(a \otimes h)=a_{(0)} \otimes h_{(2)}^{\gamma} \gamma\left(a_{(1)} \otimes S\left(h_{(1)}\right) h_{(3)}\right),
$$

so that for the composition $\varphi_{A, \underline{H}}^{-1} \circ \Gamma(\chi) \circ \varphi_{A, A}$ we obtain (recalling that $\Gamma(\chi)=\chi$ )

$$
\begin{aligned}
\varphi_{A, \underline{H}}^{-1} & \left(\Gamma(\chi)\left(\varphi_{A, A}\left(a \otimes_{B}^{\gamma} a^{\prime}\right)\right)\right) \\
& =\varphi_{A, \underline{H}}^{-1}\left(a_{(0)} a^{\prime}{ }_{(0)} \otimes a^{\prime}{ }_{(1)}\right) \bar{\gamma}\left(a_{(1)} \otimes a^{\prime}{ }_{(2)}\right) \\
& =a_{(0)} a^{\prime}{ }_{(0)} \otimes{ }^{\prime} a_{(3)}^{\prime} \gamma\left(a_{(1)} a^{\prime}{ }_{(1)} \otimes S\left(a_{(2)}^{\prime}\right) a_{(4)}^{\prime}\right) \bar{\gamma}\left(a_{(2)} \otimes a_{(5)}^{\prime}\right) \\
& =a_{(0)} a^{\prime}{ }_{(0)} \otimes{ }^{\gamma} a^{\prime}{ }_{(4)} \bar{\gamma}\left(a_{(1)} \otimes a_{(1)}\right) u_{\gamma}\left(a^{\prime}{ }_{(2)}\right) \bar{\gamma}\left(S\left(a^{\prime}{ }_{(3)}\right) \otimes a_{(5)}^{\prime}\right) \gamma\left(a_{(2)} \otimes a_{(6)}^{\prime}\right) \bar{\gamma}\left(a_{(3)} \otimes a_{(7)}^{\prime}\right),
\end{aligned}
$$

where we have used (3.7). The last two terms simplify, giving the desired identity.

From the properties of the canonical map (Proposition 2.10) and from Proposition 2.21 it immediately follows that all arrows in the diagram are $A_{\gamma} \mathcal{M}^{H_{\gamma}}$-morphisms. In 3.1.1 below we introduce a right $A_{\gamma}$-module structure on $A_{\gamma} \otimes^{\gamma} \underline{H}_{\gamma}$ such that $A_{\gamma} \otimes^{\gamma} \underline{H}_{\gamma}$ is an object in ${ }_{A_{\gamma}} \mathcal{M}_{A_{\gamma}}{ }^{H_{\gamma}}$, and show that all arrows in the diagram are also morphisms in ${ }_{A_{\gamma}} \mathcal{M}_{A_{\gamma}}{ }^{H_{\gamma}}$.

Corollary 3.7. $B=A^{\mathrm{coH}} \subseteq A$ is an H-Hopf-Galois extension if and only if $B \simeq A_{\gamma}^{\mathrm{coH}_{\gamma}} \subseteq A_{\gamma}$ is an $H_{\gamma}$-Hopf-Galois extension. Moreover, $B \subseteq A$ is cleft if and only if $B \subseteq A_{\gamma}$ is cleft.

Proof. The main statement follows from the invertibility of the morphisms $\varphi_{A, H}, \varphi_{A, A}$ and $(5$ in diagram (3.12). For the statement about cleftness, recall, from the end of $\$ 2.1$, that the Hopf-Galois extension $B \subseteq A$ is cleft if and only if there exists an isomorphism $\theta: B \otimes H \rightarrow A$ of left $B$-modules and right $H$-comodules, where here $B \otimes H$ is the object in ${ }_{B} \mathcal{M}^{H}$ with left $B$-action given by $m_{B} \otimes$ id and right $H$-coaction given by id $\otimes \Delta$. Now, due to the functor 


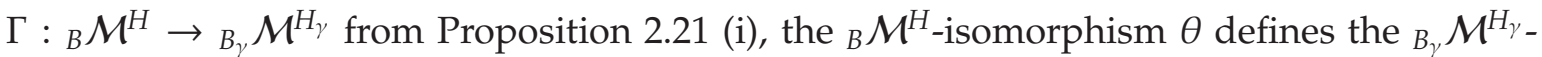
isomorphism $\Gamma(\theta):(B \otimes H)_{\gamma} \rightarrow A_{\gamma}$, which composed with the $B_{\gamma} \mathcal{M}^{H_{\gamma}}$-isomorphism $\varphi_{B, H}$

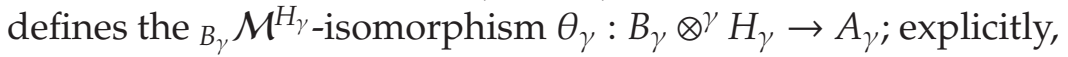

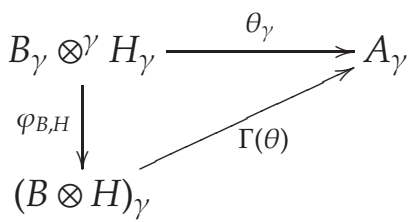

Notice that since on $B=A^{c o H} \subseteq A$ the $H$-coaction is trivial, it follows that $\varphi_{B, H}=$ id and as $\mathbb{K}$-linear maps $\theta_{\gamma}=\theta$.

Remark 3.8. Montgomery and Schneider in [27, Th. 5.3] prove the above corollary by using that as $\mathbb{K}$-modules $A \otimes_{B} A=A_{\gamma} \otimes_{B} A_{\gamma}$ and $A \otimes H=A_{\gamma} \otimes H_{\gamma}$, and showing that the canonical map $\chi$ is the composition of $\chi_{\gamma}$ with an invertible map. The proof is not within the natural categorical setting of twists of Hopf-Galois extensions that we consider.

Recalling from Definition 2.14 the notion of principal $H$-comodule algebra it is easy to show that deformations by 2-cocycles $\gamma: H \otimes H \rightarrow \mathbb{K}$ preserve this structure.

Corollary 3.9. $A$ is a principal $H$-comodule algebra if and only if $A_{\gamma}$ is a principal $H_{\gamma}$-comodule algebra.

Proof. The $B_{\gamma} \mathcal{M}^{H_{\gamma}}$-morphism $m_{\gamma}: B_{\gamma} \otimes^{\gamma} A_{\gamma} \rightarrow A_{\gamma}$ is related to the ${ }_{B} \mathcal{M}^{H}$-morphism $m: B \otimes A \rightarrow$ $A$ via $m_{\gamma}=\Gamma(m) \circ \varphi_{B, A}$. Given now a ${ }_{B} \mathcal{M}^{H}$-morphism $s: A \rightarrow B \otimes A$ that is a section of $m$, we define the ${ }_{B_{\gamma}} \mathcal{M}^{H_{\gamma}}$-morphism $s_{\gamma}:=\varphi_{B, A}^{-1} \circ \Gamma(s): A_{\gamma} \rightarrow B_{\gamma} \otimes A^{\gamma} A_{\gamma}$. We obtain the commutative diagram

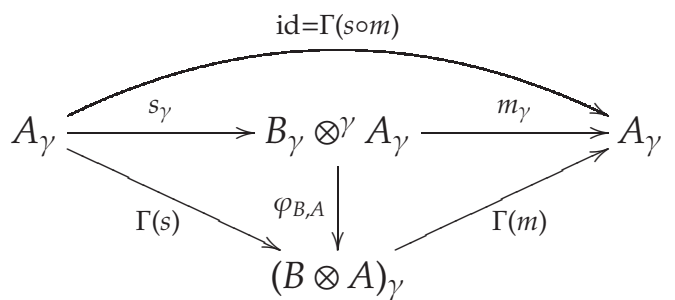

in the category ${ }_{B}, \mathcal{M}^{H_{\gamma}}$, from which it follows that $s_{\gamma}$ is a section of $m_{\gamma}$. The reverse implication follows using the convolution inverse $\bar{\gamma}$ of $\gamma$ that twists back $A_{\gamma}$ to $A$ and $(B \otimes A)_{\gamma}$ to $B \otimes A$, so that, given the section $s_{\gamma}$ of $m_{\gamma}$, the section of $m$ is $\bar{\Gamma}\left(\varphi_{B, A} \circ s_{\gamma}\right)=\varphi_{B, A} \circ s_{\gamma}: A \rightarrow B \otimes A$.

\subsubsection{Completion of the proof of Theorem 3.6 (the right $A_{\gamma}$-module structure on $A_{\gamma} \otimes^{\gamma} \underline{H}_{\gamma}$ )}

We here complete the proof of Theorem 3.6 i.e., we show that the diagram (3.12) is a diagram in the category ${ }_{A_{\gamma}} \mathcal{M}_{A_{\gamma}}{ }^{H_{\gamma}}$ (not just in ${ }_{A_{\gamma}} \mathcal{M}^{H_{\gamma}}$ ). This is the case if all morphisms in (3.12) are in ${ }_{A_{\gamma}} \mathcal{M}_{A_{\gamma}}{ }^{H_{\gamma}}$. By Proposition 2.10 it is clear that the morphism $\chi_{\gamma}$ is in ${ }_{A_{\gamma}} \mathcal{M}_{A_{\gamma}}{ }^{H_{\gamma}}$, and using Proposition 2.21(iii) we observe that also $\varphi_{A, A}$ and $\Gamma(\chi)$ are morphisms in $A_{\gamma} \mathcal{M}_{A_{\gamma}} H_{\gamma}$. In order to prove that the remaining morphisms id $\otimes^{\gamma}\left(\mathfrak{5}\right.$ and $\varphi_{A, \underline{H}}$ in the right column in (3.12) are 
morphisms in ${ }_{A_{\gamma}} \mathcal{M}_{A_{\gamma}}{ }^{H_{\gamma}}$, we just have to introduce a right $A_{\gamma}$-module structure on $A_{\gamma} \otimes \gamma \underline{H}_{\gamma}$ and prove that these morphisms are morphisms of right $A_{\gamma}$-modules (Lemma 3.11). Indeed,

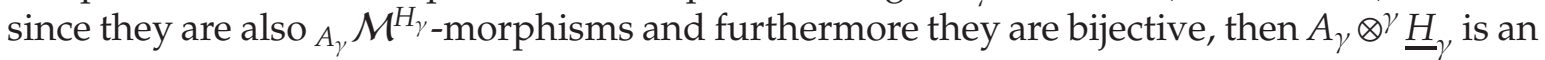
object in ${ }_{A_{\gamma}} \mathcal{M}_{A_{\gamma}}{ }^{H_{\gamma}}$ because it is the target of the first (or equivalently because it is the source

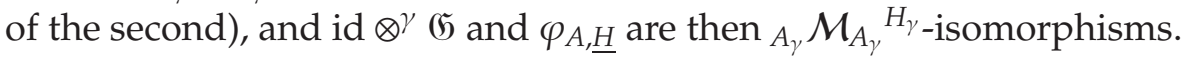

We are therefore left to introduce a right $A_{\gamma}$-module structure on $A_{\gamma} \otimes^{\gamma} \underline{H}_{\gamma}$ and prove that $\mathrm{id} \otimes^{\gamma}\left(5\right.$ and $\varphi_{A, H}$ are right $A_{\gamma}$-modules morphisms (Lemma 3.11). To this aim let us recall that the right $A$-action on $A \otimes \underline{H}$ is given by $(a \otimes h) \triangleleft A \otimes \underline{H} c=a c_{(0)} \otimes h c_{(1)}$, for all $a, c \in A$ and $h \in \underline{H}$ (cf. (2.16)). Applying Proposition 2.21(iii), we observe that the right $A_{\gamma}$-module structure on $(A \otimes \underline{H})_{\gamma}$ is given by

$$
(a \otimes h) \triangleleft(A \otimes \underline{H})_{\gamma} c=a_{(0)} c_{(0)} \otimes h_{(2)} c_{(1)} \bar{\gamma}\left(a_{(1)} S\left(h_{(1)}\right) h_{(3)} \otimes c_{(2)}\right),
$$

for all $a \in A, h \in \underline{H}$ and $c \in A_{\gamma}$. Again by (2.16), the right $A_{\gamma}$-module structure on $A_{\gamma} \otimes \gamma H_{\gamma}$ reads

$$
\left(a \otimes^{\gamma} h\right) \triangleleft A_{\gamma} \otimes^{\gamma} \underline{H_{\gamma}} c=a \bullet \gamma c_{(0)} \otimes^{\gamma} h \cdot \gamma c_{(1)},
$$

for all $a, c \in A_{\gamma}$ and $h \in H_{\gamma}$. The right $A_{\gamma}$-module structure on $A_{\gamma} \otimes^{\gamma} \underline{H}_{\gamma}$ is induced by the Hopf algebra structure on $\underline{H}_{\gamma}$ that is inherited from the Hopf algebra structure on $H_{\gamma}$ and the isomorphism 5 of Theorem 3.4 Explicitly, we have the following Corollary of Theorem 3.4 .

Corollary 3.10. The right $H_{\gamma}$-comodule coalgebra isomorphism $\left(5: H_{\gamma} \rightarrow \underline{H}_{\gamma}\right.$ defines the $\mathbb{K}$-linear isomorphism $15: H_{\gamma} \rightarrow \underline{H}_{\gamma}$ (with slight abuse of notation we use the same letter (5) that induces on $\underline{H}_{\gamma}$ a Hopf algebra structure from the Hopf algebra structure on $H_{\gamma}$. Explicitly, we define the product $m_{\underline{H}_{\gamma}}$ on $\underline{H}_{\gamma}$ via the commutative diagram of $\mathbb{K}$-linear maps

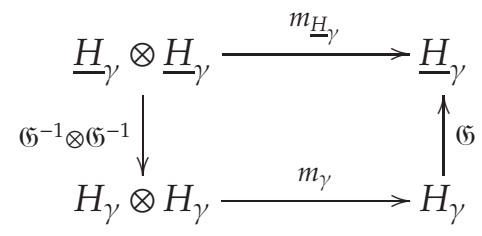

and the antipode $\underline{S}_{\underline{H}_{\gamma}}$ on $\underline{H}_{\gamma}$ via the commutative diagram of $\mathbb{K}$-linear maps

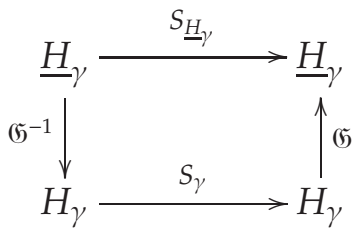

By construction, $\mathrm{H}_{\gamma}$ and $\underline{H}_{\gamma}$ are isomorphic Hopf algebras via the isomorphism $(\mathfrak{5}$.

As a simple consequence of this corollary, every right $H_{\gamma}$-comodule is also a right $\underline{H}_{\gamma}$ comodule; just use the isomorphism $(5)$ between the Hopf algebras $H_{\gamma}$ and $\underline{H}_{\gamma}$ in order to turn a right $H_{\gamma}$-comodule structure into a right $\underline{H}_{\gamma}$-comodule structure. In particular, we have 
that the right $H_{\gamma}$-comodule algebra $A_{\gamma}$ is a right $\underline{H}_{\gamma}$-comodule algebra with coaction given by

$$
\begin{aligned}
\underline{\delta}^{A_{\gamma}}:=\left(\mathrm{id} \otimes(\mathfrak{5}) \circ \delta^{A_{\gamma}}: A_{\gamma}\right. & \longrightarrow A_{\gamma} \otimes \underline{H}_{\gamma}, \\
a & \longmapsto a_{(0)} \otimes \mathfrak{5}\left(a_{(1)}\right) .
\end{aligned}
$$

Using this right $\underline{H}_{\gamma}$-comodule structure on $A_{\gamma}$ we canonically define the right $A_{\gamma}$-module structure on $A_{\gamma} \otimes^{\gamma} \underline{H}_{\gamma}$ by (cf. (2.16)),

$$
\left(a \otimes{ }^{\gamma} h\right) \triangleleft_{A_{\gamma} \otimes \underline{H}_{\gamma}^{\gamma}} c=a \bullet \gamma \mathcal{c}_{(0)} \otimes^{\gamma} m_{\underline{H}_{\gamma}}\left(h \otimes \mathfrak{G}\left(c_{(1)}\right)\right) .
$$

Lemma 3.11. The vertical arrows $\mathrm{id} \otimes{ }^{\gamma}\left(5\right.$ and $\varphi_{A, \underline{H}}$ in diagram (3.12) are right $A_{\gamma}$-module morphisms.

Proof. Using (3.14), (3.18) and (3.15) we immediately obtain that id $\otimes \gamma(5$ is a morphism of right $A_{\gamma}$-modules:

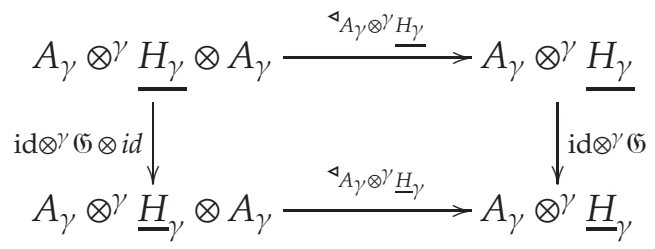

We now show that $\varphi_{A, \underline{H}}: A_{\gamma} \otimes^{\gamma} \underline{H}_{\gamma} \rightarrow(A \otimes \underline{H})_{\gamma}$ is a morphism of right $A_{\gamma}$-modules. (This is automatic in the case of Hopf-Galois extension because of the invertibility of all maps in the commutative diagram (3.12)).

The structure of right $A_{\gamma}$-modules of $A_{\gamma} \otimes^{\gamma} \underline{H}_{\gamma}$ was given just above in (3.18). We compute it explicitly by using the expression of the product in $\underline{H}_{\gamma}$ and of the map ${\left(5^{-1}\right.}^{\text {: }}$

$$
\begin{aligned}
(a \otimes h) \triangleleft A_{\gamma} \otimes \underline{H}_{\gamma} c & =a \bullet \gamma c_{(0)} \otimes m_{\underline{H}_{\gamma}}\left(h \otimes\left(\mathfrak{5}\left(c_{(1)}\right)\right)\right. \\
\quad & =a \bullet \gamma c_{(0)} \otimes\left(5\left(h_{(4)} c_{(2)}\right) \gamma\left(h_{(3)} \otimes c_{(1)}\right) \bar{\gamma}\left(h_{(5)} \otimes c_{(3)}\right) \bar{u}_{\gamma}\left(h_{(2)}\right) \gamma\left(S\left(h_{(1)}\right) \otimes h_{(6)}\right)\right. \\
& =a \bullet \gamma c_{(0)} \otimes h_{(6)} c_{(4)} \gamma\left(h_{(4)} c_{(2)} \otimes S\left(c_{(3)}\right) S\left(h_{(5)}\right) h_{(7)} c_{(5)}\right) \gamma\left(h_{(3)} \otimes c_{(1)}\right) \bar{u}_{\gamma}\left(h_{(2)}\right) \bar{\gamma}\left(h_{(8)} \otimes c_{(6)}\right) \gamma\left(S\left(h_{(1)}\right) \otimes h_{(9)}\right)
\end{aligned}
$$

where in the last passage we used the expression, $\left(\mathfrak{5}(g)=g_{(3)} \gamma\left(g_{(1)} \otimes S\left(g_{(2)}\right) g_{(4)}\right)\right.$, for all $g \in H$, that immediately follows from the definition of $(5)$ using (3.5). Now we apply (3.6) in the form $\gamma\left(h_{(3)} \otimes c_{(1)}\right) \bar{u}_{\gamma}\left(h_{(2)}\right)=\bar{\gamma}\left(S\left(h_{(2)}\right) \otimes h_{(3)} c_{(1)}\right)$ and then, by applying Lemma2.16 (iii) to the resulting term

$$
\gamma\left(h_{(4)} \mathcal{C}_{(2)} \otimes S\left(c_{(3)}\right) S\left(h_{(5)}\right) h_{(7)} \mathcal{C}_{(5)}\right) \bar{\gamma}\left(S\left(h_{(2)}\right) \otimes h_{(3)} c_{(1)}\right)
$$

the above expression becomes

$$
\begin{aligned}
& a \bullet \gamma c_{(0)} \otimes h_{(6)} c_{(5)} \gamma\left(c_{(1)} \otimes S\left(c_{(4)}\right) S\left(h_{(5)}\right) h_{(7)} c_{(6)}\right) \bar{\gamma}\left(S\left(h_{(2)}\right) \otimes h_{(3)} c_{(2)} S\left(c_{(3)}\right) S\left(h_{(4)}\right) h_{(8)} c_{(7)}\right) \gamma\left(S\left(h_{(1)}\right) \otimes h_{(10)}\right) \\
& \bar{\gamma}\left(h_{(9)} \otimes c_{(8)}\right) \\
& =a \bullet \gamma c_{(0)} \otimes h_{(4)} c_{(3)} \gamma\left(c_{(1)} \otimes S\left(c_{(2)}\right) S\left(h_{(3)}\right) h_{(5)} c_{(4)}\right) \bar{\gamma}\left(S\left(h_{(2)}\right) \otimes h_{(6)} c_{(5)}\right) \gamma\left(S\left(h_{(1)}\right) \otimes h_{(8)}\right) \bar{\gamma}\left(h_{(7)} \otimes c_{(6)}\right) \\
& =a \bullet \gamma c_{(0)} \otimes h_{(5)} c_{(3)} \gamma\left(c_{(1)} \otimes S\left(c_{(2)}\right) S\left(h_{(4)}\right) h_{(6)} c_{(4)}\right) \bar{\gamma}\left(S\left(h_{(3)}\right) \otimes h_{(7)} c_{(5)}\right) \gamma\left(S\left(h_{(2)}\right) \otimes h_{(8)} c_{(6)}\right) \bar{\gamma}\left(S\left(h_{(1)}\right) h_{(9)} \otimes c_{(7)}\right)
\end{aligned}
$$

where in the last passage we have used the cocycle condition (iv). Finally by simplifying the convolution product term $\bar{\gamma}\left(S\left(h_{(3)}\right) \otimes h_{(7)} \mathcal{C}_{(5)}\right) \gamma\left(S\left(h_{(2)}\right) \otimes h_{(8)} \mathcal{C}_{(6)}\right)$ we obtain

$$
(a \otimes h) \triangleleft_{A_{\gamma} \otimes \underline{H}_{\gamma}} c=a \bullet \gamma c_{(0)} \otimes h_{(3)} c_{(3)} \gamma\left(c_{(1)} \otimes S\left(c_{(2)}\right) S\left(h_{(2)}\right) h_{(4)} c_{(4)}\right) \bar{\gamma}\left(S\left(h_{(1)}\right) h_{(5)} \otimes c_{(5)}\right) .
$$


The invertible map $\varphi_{A, \underline{H}}$ is a right $A_{\gamma}$-modules isomorphism if this expression coincides with $\varphi_{A, \underline{H}}^{-1}\left(\left(\varphi_{A, \underline{H}}(a \otimes h)\right) \triangleleft(A \otimes \underline{H})_{\gamma} c\right)$. Recalling the right $A_{\gamma}$-module structure of $(A \otimes \underline{H})_{\gamma}$, explicitly given in (3.13), we have

$$
\begin{aligned}
\varphi_{A, \underline{H}}^{-1}\left(\left(\varphi_{A, \underline{H}}(a \otimes h)\right) \triangleleft(A \otimes \underline{H})_{\gamma} c\right)=\bar{\gamma}\left(a_{(2)} \otimes S\left(h_{(1)}\right) h_{(5)}\right) \varphi_{A, \underline{H}}^{-1}\left(a_{(0)} c_{(0)} \otimes h_{(3)} c_{(1)}\right) \bar{\gamma}\left(a_{(1)} S\left(h_{(2)}\right) h_{(4)} \otimes c_{(2)}\right) \\
=\bar{\gamma}\left(a_{(3)} \otimes S\left(h_{(1)}\right) h_{(7)}\right) \gamma\left(a_{(1)} c_{(1)} \otimes S\left(c_{(2)}\right) S\left(h_{(3)}\right) h_{(5)} c_{(4)}\right) a_{(0)} c_{(0)} \otimes h_{(4)} c_{(3)} \bar{\gamma}\left(a_{(2)} S\left(h_{(2)}\right) h_{(6)} \otimes c_{(5)}\right) .
\end{aligned}
$$

By using (3.7) to expand the term $\gamma\left(a_{(1)} c_{(1)} \otimes S\left(c_{(2)}\right) S\left(h_{(3)}\right) h_{(5)} c_{(4)}\right)$, the above expression becomes

$$
\begin{array}{r}
a_{(0)} c_{(0)} \bar{\gamma}\left(a_{(1)} \otimes c_{(1)}\right) \otimes h_{(5)} c_{(4)} u_{\gamma}\left(c_{(2)}\right) \bar{\gamma}\left(S\left(c_{(3)}\right) \otimes S\left(h_{(4)}\right) h_{(6)} c_{(5)}\right) \gamma\left(a_{(2)} \otimes S\left(h_{(3)}\right) h_{(7)} c_{(6)}\right) \bar{\gamma}\left(a_{(3)} S\left(h_{(2)}\right) h_{(8)} \otimes c_{(7)}\right) \\
\bar{\gamma}\left(a_{(4)} \otimes S\left(h_{(1)}\right) h_{(9)}\right) \\
=a_{(0)} c_{(0)} \bar{\gamma}\left(a_{(1)} \otimes c_{(1)}\right) \otimes h_{(5)} c_{(4)} u_{\gamma}\left(c_{(2)}\right) \bar{\gamma}\left(S\left(c_{(3)}\right) \otimes S\left(h_{(4)}\right) h_{(6)} c_{(5)}\right) \bar{\gamma}\left(S\left(h_{(3)}\right) h_{(7)} \otimes c_{(6)}\right) \gamma\left(a_{(2)} \otimes S\left(h_{(2)}\right) h_{(8)}\right) \\
=a_{(0)} c_{(0)} \bar{\gamma}\left(a_{(1)} \otimes c_{(1)}\right) \otimes h_{(3)} c_{(4)} u_{\gamma}\left(c_{(2)}\right) \bar{\gamma}\left(S\left(c_{(3)}\right) \otimes S\left(h_{(2)}\right) h_{(4)} c_{(5)}\right) \bar{\gamma}\left(S\left(h_{(1)}\right) h_{(5)} \otimes c_{(6)}\right) \\
=a \bullet \gamma c_{(0)} \otimes h_{(3)} c_{(3)} u_{\gamma}\left(c_{(1)}\right) \bar{\gamma}\left(S\left(c_{(2)}\right) \otimes S\left(h_{(2)}\right) h_{(4)} c_{(4)}\right) \bar{\gamma}\left(S\left(h_{(1)}\right) h_{(5)} \otimes c_{(5)}\right)
\end{array}
$$

where in the second step we have used Lemma 2.16 (iv). Finally, by using (3.5) the expression of $\varphi_{A, \underline{H}}^{-1}\left(\left(\varphi_{A, \underline{H}}(a \otimes h)\right) \triangleleft(A \otimes \underline{H})_{\gamma} c\right)$ coincides with $\underline{3.20}$ ) above.

\subsection{Deformation via a 2-cocycle based on an external symmetry $K$}

In this section we first define the notion of external symmetry (with Hopf algebra $K$ ) of an H-Hopf-Galois extension, and study the corresponding category. Then we deform this extension with a 2-cocycle on $K$.

Consider a Lie group $L$ acting via diffeomorphisms on both the total manifold and the base manifold of a bundle $P \rightarrow M$, these actions being compatible with the bundle projection (hence $L$ acts via automorphisms of $P \rightarrow M$ ). We say that $L$ is an external symmetry of $P \rightarrow M$. Considering algebras rather than manifolds (cf. Example 2.13), we term a Hopf algebra $K$ an external symmetry of the extension $B \subset A$, if $A$ is a (left) $K$-comodule algebra with $B$ a K-subcomodule algebra. If we consider principal $G$-bundles $P \rightarrow M$ then we also require $G$-equivariance of the $L$-action on the total manifold leading to algebras $A$ that are $(K, H)$-bicomodules algebras, whose category is denoted ${ }^{K} \mathcal{A}^{H}$ and defined in $\$ 2.2 .4$ before Proposition 2.27

We are thus led to term a Hopf algebra $K$ an external symmetry of an $H$-Hopf Galois extension $B=A^{c O H} \subseteq A$, if $A \in K \mathcal{A}^{H}$ and if $B=A^{c O H}$ is a $K$-subcomodule. It immediately follows that $B=A^{c o H}$ is a $(K, H)$-subbicomodule algebra.

The requirement that $B=A^{c o H}$ is a $K$-subcomodule of $A$ holds automatically true in particular if $K$ is a flat module. We recall that $K$ is a flat $\mathbb{K}$-module if any short exact sequence of $\mathbb{K}$-modules $0 \rightarrow U \stackrel{i}{\rightarrow} V \stackrel{j}{\rightarrow} W \rightarrow 0$ implies the short exact sequence of $\mathbb{K}$-modules $0 \rightarrow K \otimes U \stackrel{\mathrm{id}_{K} \otimes i}{\longrightarrow} K \otimes V \stackrel{\mathrm{id}_{K} \otimes j}{\longrightarrow} K \otimes W \rightarrow 0$. In particular all modules are flat if $\mathbb{K}$ is a field or the ring of formal power series with coefficients in a field.

Proposition 3.12. Let $H$ and $K$ be Hopf algebras, let $K$ be flat as $\mathbb{K}$-module, and let $A \in{ }^{K} \mathcal{A}^{H}$; then $B=A^{c o H}$ is a K-subcomodule algebra. 
Proof. The short exact sequence $0 \longrightarrow A^{\mathrm{coH}} \stackrel{i}{\longrightarrow} A \stackrel{\delta^{A}-\mathrm{id}_{A} \otimes \eta_{H}}{\longrightarrow} \operatorname{Im}\left(\delta^{A}-\mathrm{id}_{A} \otimes \eta_{H}\right) \longrightarrow 0$, where $\mathrm{id}_{A} \otimes \eta_{H}: A \otimes \mathbb{K} \simeq A \rightarrow A \otimes H, a \mapsto a \otimes 1_{H}$, defines the algebra of $H$-coinvariants $A^{c o H}$. If $K$ is flat we have the associated short exact sequence

$$
0 \longrightarrow K \otimes A^{c o H} \stackrel{\mathrm{id}_{K} \otimes i}{\longrightarrow} K \otimes A \stackrel{\mathrm{id}_{K} \otimes\left(\delta^{A}-\mathrm{id}_{A} \otimes \eta_{H}\right)}{\longrightarrow} K \otimes \operatorname{Im}\left(\delta^{A}-\mathrm{id}_{A} \otimes \eta_{H}\right) \longrightarrow 0
$$

Now the compatibility between the $H$ - and $K$-coactions $\delta^{A}$ and $\rho^{A}$ (cf. (2.44) implies that, for all $b \in A^{c o H}$, we have $\left(\operatorname{id}_{K} \otimes \delta^{A}\right)\left[\rho^{A}(b)\right]=\left(\rho^{A} \otimes \operatorname{id}_{H}\right)\left[\delta^{A}(b)\right]=\rho^{A}(b) \otimes 1_{H}=\left(\operatorname{id}_{K} \otimes \operatorname{id}_{A} \otimes \eta_{H}\right)\left[\rho^{A}(b)\right]$ and therefore $\rho^{A}\left(A^{c o H}\right) \in \operatorname{ker}\left[\mathrm{id}_{K} \otimes\left(\delta^{A}-\mathrm{id}_{A} \otimes \eta_{H}\right)\right]=K \otimes A^{c o H}$, where the last equality is due to the exact sequence. This proves that $B=A^{\mathrm{coH}}$ is a $K$-subcomodule of $A$, and hence a K-subcomodule algebra.

Consider now an object $A$ in ${ }^{K} \mathcal{A}^{H}$, with right $H$-coaction denoted by $\delta^{A}: A \rightarrow A \otimes H, a \mapsto$ $a_{(0)} \otimes a_{(1)}$ and left $K$-coaction by $\rho^{A}: A \rightarrow K \otimes A, a \mapsto a_{(-1)} \otimes a_{(0)}$. We trivially have $\underline{H} \in{ }^{K} \mathcal{A}^{H}$ with the $K$-coaction $\rho \underline{H}: \underline{H} \rightarrow K \otimes \underline{H}, h \mapsto 1_{K} \otimes h$. Since the category of $(K, H)$-bicomodules ${ }^{K} \mathcal{M}^{H}$ is a monoidal category and $A, \underline{H}$ are in particular objects in ${ }^{K} \mathcal{M}^{H}$, then $A \otimes A$ and $A \otimes \underline{H}$ are objects in ${ }^{K} \mathcal{M}^{H}$. Moreover $A \otimes A$ and $A \otimes \underline{H} \in{ }^{K}{ }_{A} \mathcal{M}_{A}{ }^{H}$ since the left and the right $A$-actions are $K$-comodule morphisms, indeed we easily prove commutativity of the diagrams:
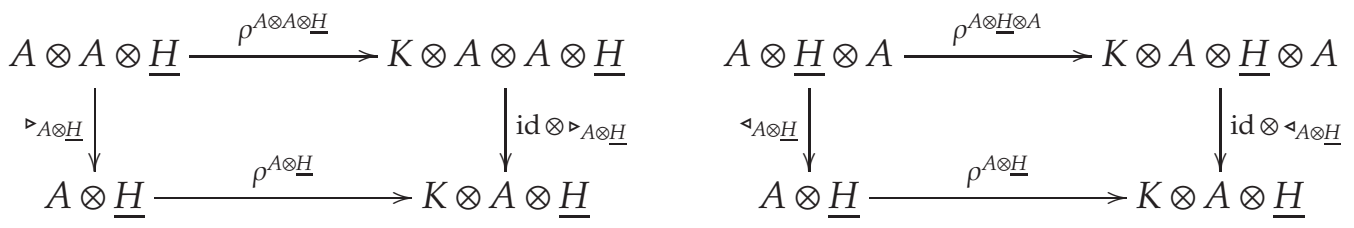

and of the corresponding ones for $A \otimes A$ (the proof that $A \otimes \underline{H}$ and $A \otimes A \in{ }_{A} \mathcal{M}$ and that $A \otimes A \in{ }^{K} \mathcal{M}_{A}$ can be also seen to follow from the property that ${ }^{K}{ }_{A} \mathcal{M}$ and ${ }^{K} \mathcal{M}_{A}$ are respectively right and left module categories over the monoidal category ${ }^{K} \mathcal{M}$ ).

Furthermore, since $B$ is a $K$-subcomodule then it is easy to see that the $K$-comodule structure of $A \otimes A$ is induced on the quotient $A \otimes_{B} A$, that is therefore an object in the relative Hopf module category ${ }_{A}{ }_{A} \mathcal{M}_{A}{ }^{H}$. We have thus proven the following

Proposition 3.13. Let $H$ and $K$ be Hopf algebras, $A \in K^{K} \mathcal{A}^{H}$ and $B=A^{c o H}$ be a K-subcomodule. Then $A \otimes_{B} A$ and $A \otimes \underline{H}$ are objects in ${ }_{A} \mathcal{M}_{A}{ }^{H}$.

Explicitly the $K$-coactions on $A \otimes_{B} A$ and on $A \otimes \underline{H}$ read

$$
\rho^{A \otimes_{B} A}: A \otimes_{B} A \longrightarrow K \otimes\left(A \otimes_{B} A\right), a \otimes_{B} c \longmapsto a_{(-1)} c_{(-1)} \otimes\left(a_{(0)} \otimes_{B} c_{(0)}\right)
$$

and

$$
\rho^{A \otimes \underline{H}}: A \otimes \underline{H} \longrightarrow K \otimes A \otimes \underline{H}, a \otimes h \longmapsto a_{(-1)} \otimes a_{(0)} \otimes h .
$$

The canonical map preserves this additional structure:

Proposition 3.14. If $A \in K^{K} \mathcal{A}^{H}$ and $B=A^{c o H}$ is a K-subcomodule, then the canonical map $\chi$ : $A \otimes_{B} A \rightarrow A \otimes \underline{H}$ is a morphism in ${ }^{K}{ }_{A} \mathcal{M}_{A}{ }^{H}$. 
Proof. Recalling from Proposition 2.10 that the canonical map $\chi$ is a morphisms in ${ }_{A} \mathcal{M}_{A}{ }^{H}$, we just have to show that it preserves the left $K$-coactions, i.e. $\rho^{A \otimes H} \circ \chi=(\mathrm{id} \otimes \chi) \circ \rho^{A \otimes_{B} A}$. This indeed holds true:

$$
\begin{aligned}
\rho^{A \otimes \underline{H}}\left(\chi\left(a \otimes_{B} c\right)\right) & =\left(a c_{(0)}\right)_{(-1)} \otimes\left(a c_{(0)}\right)_{(0)} \otimes c_{(1)} \\
& =a_{(-1)}\left(c_{(0)}\right)_{(-1)} \otimes a_{(0)}\left(c_{(0)}\right)_{(0)} \otimes c_{(1)} \\
& =a_{(-1)} c_{(-1)} \otimes a_{(0)} c_{(0)} \otimes c_{(1)} \\
& =a_{(-1)} c_{(-1)} \otimes \chi\left(a_{(0)} \otimes_{B} c_{(0)}\right) \\
& =(\mathrm{id} \otimes \chi)\left(\rho^{A \otimes_{B} A}\left(a \otimes_{B} c\right)\right),
\end{aligned}
$$

where in the third and fourth equality we have used the equivariance condition (2.45).

Let us now consider a 2-cocycle $\sigma: K \otimes K \rightarrow \mathbb{K}$ on $K$. We deform according to Proposition 2.17 the Hopf algebra $K$ into the Hopf algebra $K_{\sigma}$. Using the machinery of \$2.2.3 and \$2.2.4 we can also deform the $(K, H)$-bicomodule algebra $A$ into the $\left(K_{\sigma}, H\right)$-bicomodule algebra ${ }_{\sigma} A \in K_{\sigma} \mathcal{A}^{H}$ (choose in Proposition 2.27 the trivial 2-cocycle $\gamma\left(h \otimes h^{\prime}\right)=\varepsilon(h) \varepsilon\left(h^{\prime}\right)$ on $H$ ). If $B=A^{c o H}$ is a $K$-comodule then it is a $(K, H)$-bicomodule algebra and is as well deformed into the $\left(K_{\sigma}, H\right)$-bicomodule algebra ${ }_{\sigma} B:={ }_{\sigma}\left(A^{c o H}\right)=\left({ }_{\sigma} A\right)^{c o H} \in K_{\sigma} \mathcal{A}^{H}$. As a consequence we have the twisted canonical map ${ }_{\sigma} \chi:{ }_{\sigma} A^{\sigma} \otimes_{\sigma} B{ }_{\sigma} A \rightarrow{ }_{\sigma} A^{\sigma} \otimes \underline{H}$, which by Proposition 3.14 is a morphism in ${ }_{\sigma}{ }_{\sigma} A \mathcal{M}_{\sigma} A{ }^{H}$.

The following theorem relates the twisted canonical map ${ }_{\sigma} \chi$ with the original canonical $\operatorname{map} \chi$.

Theorem 3.15. Let $A \in{ }^{K} \mathcal{A}^{H}$ and $B=A^{c o H}$ a K-subcomodule. Given a 2-cocycle $\sigma: K \otimes K \rightarrow \mathbb{K}$ the diagram

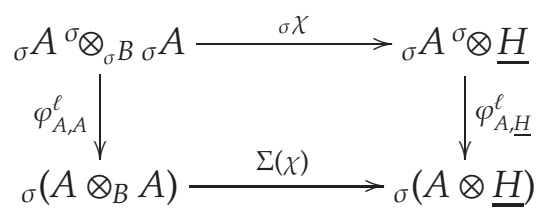

in $\mathrm{K}_{\sigma}{ }_{\sigma} A \mathcal{M}_{\sigma} A^{H}$ commutes.

Proof. First we notice that the left vertical arrow is the induction to the quotient of the isomorphism $\varphi_{A, A}^{\ell}:{ }_{\sigma} A^{\sigma} \otimes_{\sigma} A \longrightarrow{ }_{\sigma}(A \otimes A)$ defined in (2.40); it is well defined thanks to the cocycle condition (2.21) for $\sigma$. Next let us observe that $\varphi_{A, \underline{H}}^{\ell}$ is the identity; indeed, since $\underline{H}$ is equipped with the trivial left $K$-coaction $h \mapsto 1_{K} \otimes h$ and $\sigma$ is unital, we have

$$
\varphi_{A, \underline{H}^{\prime}}^{\ell}\left(a^{\sigma} \otimes h\right)=\sigma\left(a_{(-1)} \otimes h_{(-1)}\right) a_{(0)} \otimes h_{(0)}=\sigma\left(a_{(-1)} \otimes 1_{K}\right) a_{(0)} \otimes h=a \otimes h,
$$

for all $a \in{ }_{\sigma} A$ and $h \in \underline{H}$. Furthermore, it is clear by Proposition 3.14 and $\$ 2.2 .4$ that all arrows are morphisms in ${ }_{\mathrm{K}_{\sigma}}{ }_{\sigma} \mathcal{M}_{\sigma}{ }^{H}$, so it remains to prove the commutativity of the diagram:

$$
\begin{aligned}
\chi\left(\varphi_{A, A}^{\ell}\left(a^{\sigma} \otimes_{\sigma B} a^{\prime}\right)\right) & =\sigma\left(a_{(-1)} \otimes a^{\prime}{ }_{(-1)}\right) \chi\left(a_{(0)} \otimes_{B} a_{(0)}^{\prime}\right) \\
& =\sigma\left(a_{(-1)} \otimes a_{(-1)}^{\prime}\right) a_{(0)}\left(a^{\prime}{ }_{(0)}\right)_{(0)} \otimes\left(a^{\prime}{ }_{(0)}\right)_{(1)} \\
& =\sigma\left(a_{(-1)} \otimes\left(a^{\prime}{ }_{(0)}\right)_{(-1)}\right) a_{(0)}\left(a^{\prime}{ }_{(0)}\right)_{(0)} \otimes a^{\prime}{ }_{(1)} \\
& =a_{\sigma} \bullet a^{\prime}{ }_{(0)} \otimes a^{\prime}{ }_{(1)} \\
& =\sigma \chi\left(a^{\sigma} \otimes_{\sigma B} a^{\prime}\right),
\end{aligned}
$$


for all $a, a^{\prime} \in{ }_{\sigma} A$.

Corollary 3.16. $B \subseteq A$ is an H-Hopf-Galois extension if and only if ${ }_{\sigma} B \subseteq{ }_{\sigma} A$ is an H-Hopf-Galois extension.

Proof. The statement follows from the invertibility of the morphisms $\varphi_{A, \underline{H}}$ and $\varphi_{A, A}$ in diagram (3.24).

In order to prove that twist deformations of principal $H$-comodule algebras are principal $H$-comodule algebras we need the following

Proposition 3.17. Let $B \in K_{\mathcal{A}}{ }^{H}, V, W \in K_{B} \mathcal{M}^{H}$ and ${ }_{B} H_{o m}{ }^{H}(V, W)$ be the $\mathbb{K}$-module of $\mathbb{K}$-linear maps $V \rightarrow W$ that are left $B$-module maps and right $H$-comodule maps. Let $\sigma: K \otimes K \rightarrow \mathbb{K}$ be a 2-cocycle on $K$, then there is a $\mathbb{K}$-module isomorphism

$$
\begin{aligned}
\mathfrak{S}:{ }_{B} \operatorname{Hom}^{H}(V, W) & \longrightarrow{ }_{\sigma}{ }^{H} \operatorname{Hom}^{H}\left({ }_{\sigma} V_{,}{ }_{\sigma} W\right) \\
S & \longmapsto \Im(s)
\end{aligned}
$$

defined by, for all $v \in{ }_{\sigma} V$,

$$
\widetilde{S}(s)(v)=\sigma\left(v_{(-2)} \otimes S\left(v_{(-1)}\right) s\left(v_{(0)}\right)_{(-1)}\right) s\left(v_{(0)}\right)_{(0)},
$$

with inverse $\mathfrak{S}^{-1}:{ }_{\sigma} B \operatorname{Hom}^{H}\left({ }_{\sigma} V,{ }_{\sigma} W\right) \longrightarrow{ }_{B} \operatorname{Hom}^{H}(V, W), \tilde{s} \mapsto \mathfrak{\Xi}^{-1}(\tilde{s})$ given by, for all $v \in V$,

$$
\widetilde{S}^{-1}(\tilde{s})(v)=\sigma\left(S\left(v_{(-2)}\right) \otimes \tilde{S}\left(v_{(0)}\right)_{(-1)}\right) \bar{u}_{\sigma}\left(v_{(-1)}\right) \tilde{S}\left(v_{(0)}\right)_{(0)} .
$$

On K-comodule maps we have $\mathfrak{S}=\Sigma$.

Proof. Use of property (3.6) for the 2-cocycle $\sigma$ shows that an expression equivalent to (3.26) is $\widetilde{S}(s)(v)=u_{\sigma}\left(v_{(-2)}\right) \bar{\sigma}\left(S\left(v_{(-1)}\right) \otimes s\left(v_{(0)}\right)_{(-1)}\right) s\left(v_{(0)}\right)_{(0)}$. Then it is easy to directly check that (3.27) defines the inverse of $\mathfrak{\Xi}$. The $H$-comodule property of $\subseteq(s)$ is a straightforward consequence of the $H$-comodule property of $s$ and of the compatibility between the $H$ - and $K$-coactions. We now show that $\mathfrak{S}(s)$ is a left ${ }_{\sigma} B$-linear map: for all $b \in{ }_{\sigma} B, v \in{ }_{\sigma} V$,

$$
\begin{aligned}
& \mathfrak{S}(s)\left(b_{\sigma} \bullet v\right)=\sigma\left(b_{(-1)} \otimes v_{(-1)}\right) \mathfrak{S}(s)\left(b_{(0)} v_{(0)}\right) \\
& =\sigma\left(b_{(-4)} \otimes v_{(-3)}\right) \sigma\left(b_{(-3)} v_{(-2)} \otimes S\left(b_{(-2)} v_{(-1)}\right) b_{(-1)} s\left(v_{(0)}\right)_{(-1)}\right) b_{(0)} s\left(v_{(0)}\right)_{(0)} \\
& =\sigma\left(b_{(-2)} \otimes v_{(-3)}\right) \sigma\left(b_{(-1)} v_{(-2)} \otimes S\left(v_{(-1)}\right) s\left(v_{(0)}\right)_{(-1)}\right) b_{(0)} s\left(v_{(0)}\right)_{(0)} \\
& =\sigma\left(b_{(-3)} \otimes v_{(-4)}\right) \bar{\sigma}\left(b_{(-2)} \otimes v_{(-3)}\right) \sigma\left(v_{(-2)} \otimes S\left(v_{(-1)}\right) s\left(v_{(0)}\right)_{(-2)}\right) \sigma\left(b_{(-1)} \otimes s\left(v_{(0)}\right)_{(-1)}\right) b_{(0)} s\left(v_{(0)}\right)_{(0)} \\
& =\sigma\left(b_{(-1)} \otimes s\left(v_{(0)}\right)_{(-1)}\right) \sigma\left(v_{(-2)} \otimes S\left(v_{(-1)}\right) s\left(v_{(0)}\right)_{(-2)}\right) b_{(0)} s\left(v_{(0)}\right)_{(0)} \\
& =b_{\sigma} \bullet\left(\sigma\left(v_{(-2)} \otimes S\left(v_{(-1)}\right) s\left(v_{(0)}\right)_{(-1)}\right) s\left(v_{(0)}\right)_{(0)}\right) \\
& =b_{\sigma} \bullet \mathfrak{S}(s)(v)
\end{aligned}
$$

where in the second equality we used left B-linearity of $s$, and in the fourth Lemma 3.2 . Finally if $s$ is a $K$-comodule map then we immediately see that $\mathfrak{S}(s)=\Sigma(s)$. 
Remark 3.18. Consider a Hopf algebra $\mathcal{V}$ dually paired to $K$. If $V, W$ are left $K$-comodules, then they are right $\mathcal{V}$-modules and left $\mathcal{V}^{o p}$-modules; let $\triangleright_{V}$ and $\triangleright_{W}$ be the corresponding $\mathcal{V}^{o p_{-}}$ actions, cf. Appendix A.2 (recall that $\mathcal{V}^{o p}$ is the Hopf algebra with opposite product, inverse antipode and same coproduct and counit as $\mathcal{V})$. The set of $\mathbb{K}$-linear maps $\operatorname{Hom}_{\mathbb{K}}(V, W)$ is canonically a left $\mathcal{V}^{o p}$-module with the adjoint action $\mathcal{V}^{o p} \otimes \operatorname{Hom}_{\mathbb{K}}(V, W) \rightarrow \operatorname{Hom}_{\mathbb{K}}(V, W)$, $(v, s) \mapsto v \triangleright^{c o p} s:=v_{(2)} \triangleright_{W} \circ S \circ S^{\mathcal{V}^{o p}}\left(v_{(1)}\right) \triangleright_{V}$.

A twist $\mathcal{G}$ on $\mathcal{V}$ defines a 2-cocycle $\sigma$ on $K$ (cf. A.4 in Appendix A.2) and a twist $\mathcal{G}^{o p}=\mathcal{G}^{-1}$ on $\mathcal{V}^{o p}$. In this case ${ }_{\sigma} V,{ }_{\sigma} W$ are right $\mathcal{V}^{\mathcal{G}}$-modules and henceforth left $\mathcal{V}^{\mathcal{G}^{o p}}=\left(\mathcal{V}^{o p}\right)^{\mathcal{G}^{o p}}$ modules and so is $\operatorname{Hom}_{\mathbb{K}}\left({ }_{\sigma} V,{ }_{\sigma} W\right)$ with the $\mathcal{V}^{\mathcal{G}^{o p}}$-adjoint action; also ${ }_{\sigma}\left(\operatorname{Hom}_{\mathbb{K}}(V, W)\right)$ is a left $\mathcal{V}^{\mathcal{G}^{o p}}$-module. As proven in [4] Theorem 4.8, these last two are isomorphic left $\mathcal{V}^{\mathcal{G}^{o p}}$-modules via $D_{G^{o p}}^{c o p}:{ }_{\sigma}\left(\operatorname{Hom}_{\mathbb{K}}(V, W)\right) \rightarrow \operatorname{Hom}_{\mathbb{K}}\left({ }_{\sigma} V,{ }_{\sigma} W\right)$. This map is the same as that in Proposition 3.17 (with $B$ and $H$ trivial). Explicitly

$$
D_{\mathcal{G}^{o p}}^{c o p}(s)=\mathrm{g}_{\alpha_{(2)}} \triangleright_{W} \circ S \circ \mathrm{g}^{\alpha} S\left(\mathrm{~g}_{\alpha_{(1)}}\right) \triangleright_{V}=\mathfrak{S}(s),
$$

where $\mathcal{G}=\mathrm{g}^{\alpha} \otimes \mathrm{g}_{\alpha}$, and the product, coproduct and antipode are those of $\mathcal{V}$. We refer to [4] for further properties of this left deformation map.

The categorical viewpoint is also instructive. We first define the functor hom : $\left({ }^{\mathcal{V}} \mathcal{M}\right)^{o p} \times$ ${ }^{\mathcal{V}^{o p}} \mathcal{M} \rightarrow \mathcal{V}^{\text {op }} \mathcal{M}$, that on objects is given by $\operatorname{hom}(V, W)=\operatorname{Hom}_{\mathbb{K}}(V, W)$ (the $\mathcal{V}^{o p}$-module of $\mathbb{K}$ linear maps $V \rightarrow W)$, while on morphisms $V \stackrel{f}{\leftarrow} X, W \stackrel{g}{\rightarrow} Y$ it is given by $h o m(f, g)(L)=$ $g \circ L \circ f \in \operatorname{hom}(X, Y)$ for all $\mathbb{K}$-linear maps $L \in \operatorname{hom}(V, W)$. The functor hom is an internal hom functor in the monoidal category $\left({ }^{o p} \mathcal{M}, \otimes\right)$ because, for all $V$, the functor $h o m(V,-)$ is right adjoint to the tensor product functor $V \otimes-$; a proof is in [5] §2.5, where the quasi-Hopf algebra $H$ there is the Hopf algebra $\mathcal{V}^{o p}{ }^{c o p}$ (i.e., $\mathcal{V}^{o p}$ with opposite coproduct and antipode). Thus hom defines a closed monoidal category structure on $\left({ }^{\mathcal{o p}} \mathcal{M}, \otimes\right)$. Similarly we have the closed monoidal category $\left({ }^{\mathcal{V}^{\mathcal{o p}}} \mathcal{M},{ }^{\sigma} \otimes, h o m_{\sigma}\right)$. Then, in this framework, the left $\mathcal{V}^{\mathcal{G}^{o p}}$-module isomorphisms $\mathfrak{S}:{ }_{\sigma}\left(\operatorname{Hom}_{\mathbb{K}}(V, W)\right) \rightarrow \operatorname{Hom}_{\mathbb{K}}\left({ }_{\sigma} V,{ }_{\sigma} W\right)$, that could be denoted $\mathfrak{S}_{V, W}$, are the components of a natural isomorphism $\Sigma \circ$ hom $\Rightarrow h_{\sigma} m_{\sigma} \circ\left(\Sigma^{o p} \times \Sigma\right)$ between functors from the category $\left({ }^{\mathcal{V}^{o p}} \mathcal{M}\right){ }^{o p} \times \mathcal{V}^{o p} \mathcal{M}$ to the category ${ }^{\mathcal{V}}{ }^{o p} \mathcal{M}$. Via this natural isomorphism ${ }^{\mathcal{N o p}} \mathcal{M}$ and $\mathcal{V}^{\mathcal{G} p} \mathcal{M}$ are equivalent as closed monoidal categories.

Corollary 3.19. $A$ is a principal $H$-comodule algebra if and only if ${ }_{\sigma} A$ is a principal $H$-comodule algebra.

Proof. The proof is similar to that of Corollary 3.9 with the caveat that since $s$ is not a $K$ comodule map we have to consider its deformation via the map $\subseteq$. Hence we consider the commutative diagram

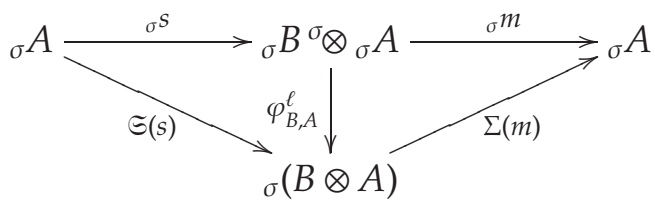

where by definition ${ }_{\sigma} S:=\subseteq(s) \circ\left(\varphi_{B, A}^{\ell}\right)^{-1}$. Left ${ }_{\sigma} B$-linearity and right $H$-colinearity of $\subseteq(s)$ and of $\varphi_{B, A}^{\ell}$ imply that also ${ }_{\sigma} S$ is a map in ${ }_{\sigma} B \mathcal{M}^{H}$. Furthermore it is a section of the (restricted) 
product ${ }_{\sigma} m:{ }_{\sigma} B^{\sigma} \otimes{ }_{\sigma} A \rightarrow{ }_{\sigma} A$ since, for all $a \in{ }_{\sigma} A$

$$
\begin{aligned}
\left({ }_{\sigma} m \circ{ }_{\sigma} s\right)(a) & =(\Sigma(m) \circ \mathbb{S}(s))(a) \\
& =\sigma\left(a_{(-2)} \otimes S\left(a_{(-1)}\right) s\left(a_{(0)}\right)_{(-1)}\right) m\left(s\left(a_{(0)}\right)_{(0)}\right) \\
& =\sigma\left(a_{(-2)} \otimes S\left(a_{(-1)}\right) m\left(s\left(a_{(0)}\right)\right)_{(-1)}\right) m\left(s\left(a_{(0)}\right)\right)_{(0)} \\
& \left.=\sigma\left(a_{(-2)} \otimes S\left(a_{(-1)}\right) a_{(0)}\right) a_{(-1)}\right) \\
& =a
\end{aligned}
$$

where in the third equality we used that $m$ is a $K$-comodule map, and in the fourth that $m \circ s=\mathrm{id}_{A}$.

Remark 3.20. Concerning cleftness of the extension ${ }_{\sigma} B \simeq{ }_{\sigma} A^{\mathrm{coH}} \subset{ }_{\sigma} A$, if $\theta: B \otimes H \rightarrow A$ is an isomorphisms of left $B$-modules, left $K$-comodules and right $H$-comodules then, as in Corollary 3.7, $\Sigma(\theta):{ }_{\sigma} B \otimes H \rightarrow{ }_{\sigma} A$ is an isomorphisms of left ${ }_{\sigma} B$-modules, left ${ }_{\sigma} K$-comodules and right $H$-comodules, with inverse $\Sigma\left(\theta^{-1}\right)$. In general however $\theta: B \otimes H \rightarrow A$ is not a left $K$-comodule map, then we can consider $\subseteq(\theta):{ }_{\sigma} B \otimes H \rightarrow{ }_{\sigma} A$ that is a map in ${ }_{\sigma} B \mathcal{M}^{H}$; if this map is invertible then cleftness of $A^{\mathrm{coH}} \subset A$ implies cleftness of ${ }_{\sigma} A^{\mathrm{coH}} \subset{ }_{\sigma} A$. In the context of formal deformation quantization this is always the case, and considering also $\subseteq^{-1}$ we obtain that $A^{\mathrm{coH}} \subset A$ is cleft if and only if ${ }_{\sigma} A^{\mathrm{coH}} \subset{ }_{\sigma} A$ is cleft.

Example 3.21 (The instanton bundle on the isospectral sphere $S_{\theta}^{4}$ [23, 8].). The classical SU(2)Hopf bundle $\pi: S^{7} \rightarrow S^{4}$ over the four-sphere $S^{4}$ can be described in different ways. We take here a pure algebraic approach well suited for the application of the deformation theory developed above.

Let $O\left(\mathbb{R}^{8}\right)$ be the commutative $*$-algebra over $\mathbb{K}=\mathbb{C}$ generated by elements $\left\{z_{i}, z_{i}^{*}, i=\right.$ $1, \ldots, 4\}$. Let $A:=O\left(S^{7}\right)$ be the algebra of coordinate functions on the 7-sphere $S^{7}$ obtained as the quotient of $O\left(\mathbb{R}^{8}\right)$ by the two-sided $*$-ideal generated by the element $\sum z_{i}^{*} z_{i}-1$. Let $H:=O(S U(2))$ be the Hopf algebra of coordinate functions on $S U(2)$ realized as the *-algebra generated by commuting elements $\left\{w_{i}, w_{i}^{*}, i=1,2\right\}$ with $\sum w_{i}^{*} w_{i}=1$ and standard Hopf algebra structure induced from the group structure of $S U(2)$.

The classical principal action of $S U(2)$ on $S^{7}$ can be described at the algebraic level by the data of the following right coaction of $O(S U(2))$ on $O\left(S^{7}\right)$ :

$$
\begin{aligned}
& \delta^{O\left(S^{7}\right)}: \quad O\left(S^{7}\right) \longrightarrow O\left(S^{7}\right) \otimes O(S U(2)) \\
& u \longmapsto u \dot{\otimes}\left(\begin{array}{cc}
w_{1} & -w_{2}^{*} \\
w_{2} & w_{1}^{*}
\end{array}\right), \quad u:=\left(\begin{array}{cccc}
z_{1} & z_{2} & z_{3} & z_{4} \\
-z_{2}^{*} & z_{1}^{*} & -z_{4}^{*} & z_{3}^{*}
\end{array}\right)^{t}
\end{aligned}
$$

where $\dot{\otimes}$ stands for the composition of $\otimes$ with the matrix multiplication. The map $\delta O\left(S^{7}\right)$ defined above on the algebra generators, and extended to the whole $O\left(S^{7}\right)$ as a $*$-algebra morphism, structures $O\left(S^{7}\right)$ as a right $O(S U(2))$-comodule algebra. As expected, the subalgebra $B:=O\left(S^{7}\right)^{\operatorname{co}(O(S U(2)))} \subset O\left(S^{7}\right)$ of coinvariants under the coaction $\delta^{O\left(S^{7}\right)}$ can be identified with the algebra of coordinate functions on the 4-sphere $S^{4}$. Indeed the entries of the matrix

$$
\mathrm{P}:=u u^{*}=\frac{1}{2}\left(\begin{array}{cccc}
1+x & 0 & \alpha & -\beta^{*} \\
0 & 1+x & \beta & \alpha * \\
\alpha^{*} & \beta^{*} & 1-x & 0 \\
-\beta & \alpha & 0 & 1-x
\end{array}\right)
$$


where

$$
\alpha:=2\left(z_{1} z_{3}^{*}+z_{2}^{*} z_{4}\right), \quad \beta:=2\left(z_{2} z_{3}^{*}-z_{1}^{*} z_{4}\right), \quad x:=z_{1} z_{1}^{*}+z_{2} z_{2}^{*}-z_{3} z_{3}^{*}-z_{4} z_{4}^{*},
$$

(and their *-conjugated $\alpha^{*}, \beta^{*}$, with $x^{*}=x$ ), form a set of generators for $B$ and from the 7-sphere relation $\sum z_{i}^{*} z_{i}=1$ it follows that they satisfy

$$
\alpha^{*} \alpha+\beta^{*} \beta+x^{2}=1
$$

Thus the subalgebra $B$ of coinvariants is isomorphic to the algebra $O\left(S^{4}\right)$ of coordinate functions on $S^{4}$. The algebra inclusion $O\left(S^{4}\right) \hookrightarrow O\left(S^{7}\right)$ dualizes the Hopf map $\pi: S^{7} \rightarrow S^{4}$. The algebra $O\left(S^{7}\right)$ is a non cleft Hopf-Galois extension of $O\left(S^{4}\right)$. Moreover, since $O(S U(2))$ is cosemisimple and has a bijective antipode, then $O\left(S^{7}\right)$ is a principal comodule algebra (recall the last paragraph of $\$ 2.1$.

We now apply the theory developed above and deform this extension of commutative algebras by using a symmetry of the classical Hopf bundle. Let $K:=O\left(\mathbb{T}^{2}\right)$ be the commutative *-Hopf algebra of functions on the 2-torus $\mathbb{T}^{2}$ with generators $t_{j}, t_{j}^{*}=t_{j}^{-1}, j=1,2$ and co-structures $\Delta\left(t_{i}\right)=t_{i} \otimes t_{i}, \varepsilon\left(t_{i}\right)=1, S\left(t_{i}\right)=t_{i}^{-1}=t_{i}^{*}$. Let $\sigma$ be the exponential 2-cocycle on $K$ which is determined by its value on the generators:

$$
\sigma\left(t_{j} \otimes t_{k}\right)=\exp \left(i \pi \Theta_{j k}\right), \quad \Theta=\frac{1}{2}\left(\begin{array}{cc}
0 & \theta \\
-\theta & 0
\end{array}\right), \quad \theta \in \mathbb{R}
$$

and extended to the whole algebra by requiring $\sigma(a b \otimes c)=\sigma\left(a \otimes c_{(1)}\right) \sigma\left(b \otimes c_{(2)}\right)$ and $\sigma(a \otimes b c)=$ $\sigma\left(a_{(1)} \otimes c\right) \sigma\left(a_{(2)} \otimes b\right)$, for all $a, b, c \in O\left(\mathbb{T}^{n}\right)$. There is a left coaction of $O\left(\mathbb{T}^{2}\right)$ on the algebra $O\left(S^{7}\right)$ : it is given on the generators as

$$
\rho^{O\left(S^{7}\right)}: O\left(S^{7}\right) \longrightarrow O\left(\mathbb{T}^{2}\right) \otimes O\left(S^{7}\right), \quad z_{i} \longmapsto \tau_{i} \otimes z_{i},
$$

where $\left(\tau_{i}\right):=\left(t_{1}, t_{1}^{*}, t_{2}, t_{2}^{*}\right)$, and it is extended to the whole of $O\left(S^{7}\right)$ as a *-algebra homomorphism. It is easy to prove that the two coactions $\delta^{O\left(S^{7}\right)}$ and $\rho^{O\left(S^{7}\right)}$ satisfy the compatibility condition (2.44), hence they structure $O\left(S^{7}\right)$ as a $\left(O\left(\mathbb{T}^{2}\right), O(S U(2))\right)$-bicomodule algebra; furthermore $O\left(S^{4}\right)$ is a $\left(O\left(\mathbb{T}^{2}\right), O(S U(2))\right)$-subbicomodule algebra as can be easily checked on its generators, or indirectly inferred from Proposition 3.12 (since vector spaces are flat). Explicitly the $O\left(\mathbb{T}^{2}\right)$-coaction reads

$$
\alpha \longmapsto t_{1} t_{2}^{*} \otimes \alpha, \quad \beta \longmapsto t_{1}^{*} t_{2}^{*} \otimes \beta, \quad x \longmapsto 1 \otimes x .
$$

We can therefore apply the theory of deformation by 2-cocycles to both the comodule algebras $O\left(S^{7}\right)$ and $O\left(S^{4}\right)$ (recall $\$ 2.2 .4$ and the discussion above Theorem 3.15). The resulting noncommutative algebras, denoted respectively by $O\left(S_{\theta}^{7}\right)$ and $O\left(S_{\theta}^{4}\right)$, are two representatives of the class of $\theta$-spheres in [14]. In particular, the classical Hopf fibration $O\left(S^{4}\right) \hookrightarrow O\left(S^{7}\right)$ described above deforms to a quantum Hopf bundle on $O\left(S_{\theta}^{4}\right) \simeq O\left(S_{\theta}^{7}\right)^{c o H}$ with undeformed structure Hopf algebra $H=O(S U(2))$. Indeed, from Corollary 3.19 , we further obtain

Proposition 3.22. The algebra $O\left(S_{\theta}^{7}\right)$ is a principal $O(S U(2))$-comodule algebra. 
The noncommutative bundle so obtained is the quantum Hopf bundle on the ConnesLandi sphere $O\left(S_{\theta}^{4}\right)$ that was originally constructed in [23], and further studied in the context of 2-cocycles deformation in [8]. The principality of the algebra inclusion $O\left(S_{\theta}^{4}\right) \subseteq O\left(S_{\theta}^{7}\right)$ was first proven in [23, §5] by explicit construction of the inverse of the canonical map. Proposition 3.22 follows instead as a straightforward result of the general theory developed in the present section (out of the principality of the underlying classical bundle).

\subsection{Combination of deformations}

We now consider the combination of the previous two constructions. This leads to HopfGalois extensions in which the structure Hopf algebra, total space and base space are all deformed.

As before, we let $H$ and $K$ be Hopf algebras and $A \in K^{K} \mathcal{A}^{H}$ a $(K, H)$-bicomodule algebra, with $B=A^{c o H}$ a $K$-subcomodule. Let $\sigma: K \otimes K \rightarrow \mathbb{K}$ and $\gamma: H \otimes H \rightarrow \mathbb{K}$ be 2-cocycles and denote by $K_{\sigma}$ and $H_{\gamma}$ the twisted Hopf algebras and by ${ }_{\sigma} A_{\gamma}:={ }_{\sigma}\left(A_{\gamma}\right)=\left({ }_{\sigma} A\right)_{\gamma} \in K_{\sigma} \mathcal{A}^{H_{\gamma}}$ the deformed $\left(K_{\sigma}, H_{\gamma}\right)$-bicomodule algebra, see $\$ 2.2 .4$. We also have the deformed $\left(K_{\sigma}, H_{\gamma}\right)$ bicomodule algebra ${ }_{\sigma} B:={ }_{\sigma} B_{\gamma} \subseteq{ }_{\sigma} A_{\gamma}$ of $H_{\gamma}$-coinvariants in ${ }_{\sigma} A_{\gamma}$. The canonical map ${ }_{\sigma} \chi_{\gamma}$ : ${ }_{\sigma} A_{\gamma}{ }^{\sigma} \otimes{ }_{\sigma}{ }_{\sigma}{ }_{\sigma} A_{\gamma} \rightarrow{ }_{\sigma} A_{\gamma}{ }^{\sigma} \otimes{ }^{\gamma} H_{\gamma}$ is a ${ }_{\sigma}^{K_{\sigma}} A_{\gamma} \mathcal{M}_{\sigma} A_{\gamma}{ }_{\gamma}$-morphism because of Proposition 3.14. There are two equivalent ways to relate it to the canonical map $\chi: A \otimes_{B} A \rightarrow A \otimes \underline{H}$. We can apply the functor $\Sigma$ to the commutative diagram (3.12) of Theorem 3.6 and then top the resulting diagram with the analogue of the commutative diagram (3.24) of Theorem 3.15, or we can first apply the functor $\Gamma$ to (3.24) and then top it with the analogue of (3.12).

Theorem 3.23. Given two 2-cocycles $\sigma: K \otimes K \rightarrow \mathbb{K}$ and $\gamma: H \otimes H \rightarrow \mathbb{K}$ the diagrams
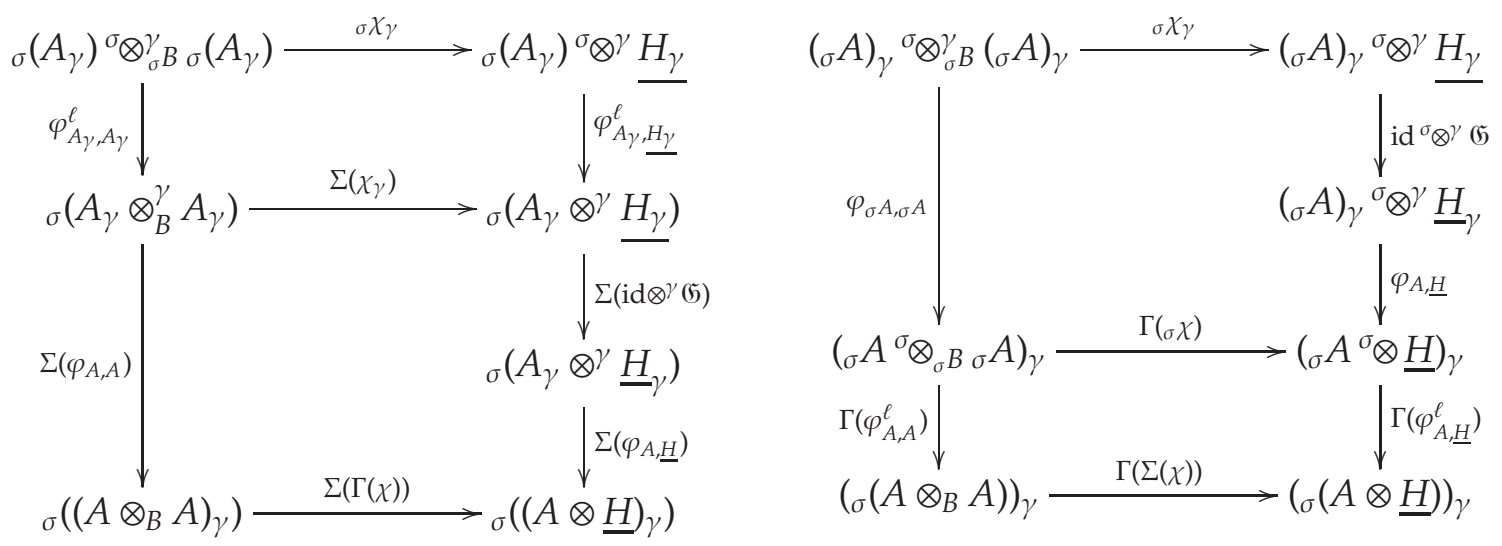

in $\mathrm{K}_{\sigma}{ }_{\sigma} A_{\gamma} \mathcal{M}_{\sigma} A_{\gamma} H_{\gamma}$ commute and have the same external square diagram. Moreover:

(i) $B \subseteq A$ is an H-Hopf-Galois extension if and only if ${ }_{\sigma} B \subseteq{ }_{\sigma} A_{\gamma}$ is an $H_{\gamma}$-Hopf-Galois extension.

(ii) $A$ is a principal $H$-comodule algebra if and only if ${ }_{\sigma} A_{\gamma}$ is a principal $H_{\gamma}$-comodule algebra.

Proof. Commutativity follows from commutativity of the internal diagrams, statements (i) and (ii) also immediately follow combining the analogue statements for each of the 2-cocycles $\gamma$ and $\sigma$. The equality of the external square diagrams follows from diagram (2.48) of Proposition 2.26 applied to the left vertical arrows, and from $\varphi_{A_{\gamma}, \underline{H_{\gamma}}}^{\ell}=\Gamma\left(\varphi_{A, \underline{H}}^{\ell}\right)=\mathrm{id}$ as well as the triviality of the functor $\Sigma$ on morphisms. 
Example 3.24 (Formal deformation quantization). Let $G$ be a Lie group, $M$ a manifold and $\pi: P \rightarrow M$ a principal $G$-bundle over $M$ with right $G$-action denoted by $r_{P}: P \times G \rightarrow P$. Then, by Example 2.13, we have a Fréchet $H=C^{\infty}(G)$-Hopf-Galois extension $B=C^{\infty}(M) \simeq$ $A^{c o H} \subseteq A=C^{\infty}(P)$ with $\mathbb{K}=\mathbb{C}$. Let us further assume that there exists another Lie group $L$ acting on the $G$-principal bundle $P \rightarrow M$, i.e. that there are left $L$-actions $l_{P}: L \times P \rightarrow P$ and $l_{M}: L \times M \rightarrow M$, such that the diagrams
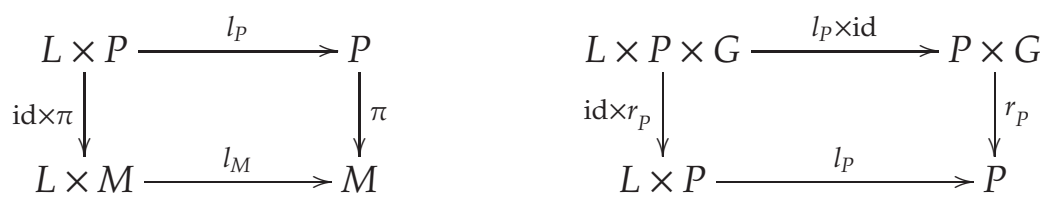

commute. For example $L$ may be a finite-dimensional Lie subgroup of the automorphism group of the bundle, which comes with a canonical left action on $P$ and $M$. Due to the left $L$-actions on $P$ and $M$ we obtain a Fréchet left $K=C^{\infty}(L)$-comodule structure on $A$ and $B$, which is compatible with the right $H$-coaction on $A$ and the canonical map because of the diagrams in (3.35), i.e. $A=C^{\infty}(P)$ is a Fréchet $\left(K=C^{\infty}(L), H=C^{\infty}(G)\right)$-bicomodule algebra.

In order to deform this example into a noncommutative Hopf-Galois extension, in the context of formal power series in a deformation parameter $\hbar$, we consider the formal power series extension of the $\mathbb{C}$-modules $H, A, B$ and $K$, denoted as usual $H[[\hbar]], A[[\hbar]], B[[\hbar]]$ and $K[[\hbar]]$. The natural topology on these $\mathbb{C}[[\hbar]]$-modules is a combination of the original Fréchet topology in each order of $\hbar$ together with the $\hbar$-adic topology, see e.g. [20, Chapter XVI]. The canonical map induces a continuous $\mathbb{C}[[\hbar]]$-linear isomorphism (denoted with abuse of notation by the same symbol)

$$
\chi: A[[\hbar]] \widehat{\otimes}_{B[[\hbar]]} A[[\hbar]] \simeq C^{\infty}\left(P \times_{M} P\right)[[\hbar]] \longrightarrow A[[\hbar]] \widehat{\otimes} \underline{H}[[\hbar]] \simeq C^{\infty}(P \times G)[[\hbar]],
$$

where now $\widehat{\otimes}$ denotes the completion of the algebraic tensor product with respect to the natural topologies described above. Hence we have obtained a topological $H[[\hbar]]-H o p f-$ Galois extension $B[[\hbar]] \simeq A[[\hbar]]^{c o H[[\hbar]]} \subseteq A[[\hbar]]$.

Notice that for $G$ a Lie group we have a (in general degenerate) pairing between the universal enveloping algebra $U(\mathfrak{g})$ of its Lie algebra $\mathfrak{g}$ and $C^{\infty}(G)$; it is determined by evaluating at the unit element $e \in G$ left invariant vector fields on functions. Explicitly, $\langle\cdot, \cdot\rangle: U(\mathfrak{g}) \times C^{\infty}(G) \rightarrow \mathbb{C}$ is defined by extending

$$
\langle 1, h\rangle:=h(e), \quad\langle v, h\rangle:=\left.\frac{d}{d t} h\left(r_{P}(e, \exp (-t v))\right)\right|_{t=0},
$$

for all $h \in C^{\infty}(G)$ and $v \in \mathfrak{g}$, to all $U(\mathfrak{g})$ via linearity and requiring $\left\langle\xi \xi^{\prime}, h\right\rangle=\left\langle\xi, h_{(1)}\right\rangle\left\langle\xi^{\prime}, h_{(2)}\right\rangle$ for all $\xi, \xi^{\prime} \in U(\mathfrak{g})$. The $\mathbb{K}$-linear maps $\langle v, \cdot\rangle: C^{\infty}(G) \rightarrow \mathbb{K}$ are continuous and since the coproduct $\Delta: C^{\infty}(G)=H \rightarrow H \widehat{\otimes} H \simeq C^{\infty}(G \times G)$ is continuous also the $\mathbb{K}$-linear maps $\langle\xi, \cdot\rangle: C^{\infty}(G) \rightarrow \mathbb{K}$ are continuous for all $\xi \in U(\mathfrak{g})$. (These maps are actually the Lie derivative along $\xi, L_{\xi}: C^{\infty}(G) \rightarrow C^{\infty}(G)$, composed with the counit in $C^{\infty}(G)$; where $L_{v}$ is the Lie derivative along the left invariant vector field defined by $v \in \mathfrak{g}$, and $L$ is extended to all $U(\mathfrak{g})$ by $\left.L_{\xi^{\prime}}=L_{\xi} \circ L_{\xi^{\prime}}\right)$. Because of this pairing we can assign to a twist $\mathcal{F}=\mathrm{f}^{\alpha} \widehat{\otimes} \mathrm{f}_{\alpha} \in$ $U(\mathfrak{g})[[\hbar]] \widehat{\otimes} U(\mathfrak{g})[[\hbar]] \simeq(U(\mathfrak{g}) \otimes U(\mathfrak{g}))[[\hbar]]$ a continuous 2-cocycle $\gamma: H[[\hbar]] \widehat{\otimes} H[[\hbar]] \rightarrow \mathbb{K}[[\hbar]]$ by defining $\gamma(h \otimes k)=\left\langle\mathbf{f}^{\alpha}, h\right\rangle\left\langle\mathrm{f}_{\alpha}, k\right\rangle$ on the dense subset $H[[\hbar]] \otimes H[[\hbar]] \subseteq H[[\hbar]] \widehat{\otimes} H[[\hbar]]$ and extending it by continuity. (See Appendix $\mathrm{A}$ for more on the duality between twists and 
2-cocycles). Similarly we may consider a twist $\mathcal{G} \in U(\mathrm{I})[[\hbar]] \widehat{\otimes} U(\mathrm{l})[[\hbar]] \simeq(U(\mathrm{I}) \otimes U(\mathrm{l}))[[\hbar]]$, where I is the Lie algebra of $L$, and define a continuous 2-cocycle $\sigma: K[[\hbar]] \widehat{\otimes} K[[\hbar]] \rightarrow \mathbb{K}[[\hbar]]$.

We now twist the $\mathbb{C}[[h]]$-modules $H[[\hbar]], A[[\hbar]], B[[\hbar]]$ and $K[[\hbar]]$ as described in general in Section 2.2, and obtain a noncommutative topological $H[[\hbar]]_{\gamma}$-Hopf-Galois extension $\left.{ }_{\sigma} B[[\hbar]] \simeq{ }_{\sigma} A[[\hbar]]_{\gamma}{ }^{c o H[[\hbar]]} \subseteq_{\sigma} A[[\hbar]]\right]_{\gamma}$. (The canonical map ${ }_{\sigma} \chi_{\gamma}$ is a continuous isomorphism, since $\chi$ and all vertical arrows in the diagrams in Theorem 3.23 are continuous isomorphisms). Recalling Remark 3.20, the Hopf-Galois extension $A[[\hbar]]^{c o H[[\hbar]]} \subseteq A[[\hbar]]$ is cleft if and only if $\left.{ }_{\sigma} A[[\hbar]]_{\gamma}^{\mathrm{coH}[[\hbar]]]_{\gamma}} \subseteq{ }_{\sigma} A[[\hbar]]\right]_{\gamma}$ is cleft.

\section{Applications}

We apply the theory so far developed first to the study of deformations of quantum homogeneous spaces in 84.1 including the explicit example of the even $\theta$-spheres $S_{\theta}^{2 n}$ in $\$ 4.1 .1$, and then to the study of deformations of sheaves of Hopf Galois extensions in $\$ 4.2$, providing the example of the Hopf bundle over $S_{\theta}^{4}$ as a twisted sheaf in 84.2 .1 .

\subsection{Twisting quantum homogeneous spaces associated with quantum subgroups}

The theory of twists, in particular the combination of deformations developed in $\$ 3.3$, can be used to study deformations of bundles over quantum homogeneous spaces arising from Hopf algebra projections. This is the subject of the present subsection.

Recall that given a Hopf algebra $G$, a quantum subgroup of $G$ is a Hopf algebra $H$ together with a surjective bialgebra (and thus Hopf algebra) homomorphism $\pi: G \rightarrow H$. The restriction via $\pi$ of the coproduct of $G$

$$
\delta^{G}:=(\mathrm{id} \otimes \pi) \circ \Delta: G \longrightarrow G \otimes H
$$

induces on $G$ the structure of a right $H$-comodule algebra. The subalgebra $B:=G^{c o H} \subseteq G$ of coinvariants is called a quantum homogeneous G-space. When the associated canonical map

$$
\chi: G \otimes_{B} G \longrightarrow G \otimes H, \quad g \otimes_{B} g^{\prime} \longmapsto g g_{(1)}^{\prime} \otimes \pi\left(g_{(2)}^{\prime}\right)
$$

is bijective, i.e. $B \subseteq G$ is a Hopf Galois-extension, we call $G$ a quantum principal bundle over the quantum homogeneous space B. (See e.g. [21, §11.6.2], [11, §5.1]).

Given a quantum principal bundle $B=G^{c o H} \subseteq G$ over a quantum homogeneous space $B$ and a 2-cocycle $\gamma$ on $H$ we can consider two different constructions:

- On the one hand we can lift the 2-cocycle $\gamma$ on $H$ to a 2-cocycle $\tilde{\gamma}$ on $G$ (see Lemma 4.1 below) and thus apply the theory of 2-cocycle deformations for Hopf algebras (\$2.2.1) to deform both $G$ and $H$ into new Hopf algebras $G_{\tilde{\gamma}}$ and $H_{\gamma}$. It turns out that the condition for $H$ to be a quantum subgroup of $G$ is preserved under deformation, i.e. $H_{\gamma}$ is a quantum subgroup of $G_{\widetilde{\gamma}}$, and thus there is an associated twisted quantum homogeneous space $B_{\tilde{\gamma}}$.

- On the other hand, we can direct the attention to the algebra inclusion $B=G^{c o H} \subseteq G$ as a Hopf-Galois extension, and twist it. In this case, we forget the Hopf algebra structure of $G$ and use $\gamma$ to deform $G$ just as an object in $\mathcal{A}^{H}$, as in $\$ 3.1$. Denote by $G_{\gamma}$ the resulting comodule algebra. 
These two deformations $G_{\widetilde{\gamma}}$ and $G_{\gamma}$ of $G$ do not coincide. In particular, $G_{\gamma}$ is not in general a Hopf algebra and thus the base space of the twisted bundle is no longer a quantum homogeneous space of the total space. Nevertheless the second construction can be reconciled with the first one by applying a further twist deformation and thus considering a combination of deformations as in $\$ 3.3$. As a corollary of this second approach we obtain that $B_{\widetilde{\gamma}} \subseteq G_{\widetilde{\gamma}}$ is a Hopf-Galois extension. Indeed we show below that, given a 2-cocycle $\gamma$ on $H$, quantum principal bundles $B=G^{c o H} \subseteq G$ over quantum homogeneous spaces $B$ deform into new quantum principal bundles over new quantum homogeneous spaces.

We proceed by first showing that given a 2-cocycle $\gamma$ on $H$ we can twist both the Hopf algebras $H$ and $G$ is such a way to still have a quantum homogeneous space.

Lemma 4.1. Let $\gamma: H \otimes H \rightarrow \mathbb{K}$ be a 2-cocycle on $H$. Then

$$
\widetilde{\gamma}: G \otimes G \longrightarrow \mathbb{K}, g \otimes g^{\prime} \longmapsto \gamma\left(\pi(g) \otimes \pi\left(g^{\prime}\right)\right)
$$

is a 2-cocycle on $\mathrm{G}$.

Proof. The proof relies on the fact that $\gamma$ is a 2-cocycle and $\pi$ is a bialgebra homomorphism, i.e. in particular $\Delta_{H} \circ \pi=(\pi \otimes \pi) \circ \Delta_{G}$ and $\varepsilon_{H} \circ \pi=\varepsilon_{G}$. We have

$$
\widetilde{\gamma}(1 \otimes g)=\gamma(1 \otimes \pi(g))=\varepsilon_{H}(\pi(g))=\varepsilon_{G}(g),
$$

and similarly $\tilde{\gamma}(g \otimes 1)=\varepsilon_{G}(g)$, for all $g \in G$. For the cocycle property (2.21) we compute

$$
\begin{aligned}
\tilde{\gamma}\left(g_{(1)} \otimes h_{(1)}\right) \tilde{\gamma}\left(g_{(2)} h_{(2)} \otimes k\right) & =\gamma\left(\pi\left(g_{(1)}\right) \otimes \pi\left(h_{(1)}\right)\right) \gamma\left(\pi\left(g_{(2)}\right) \pi\left(h_{(2)}\right) \otimes \pi(k)\right) \\
& =\gamma\left(\pi(g)_{(1)} \otimes \pi(h)_{(1)}\right) \gamma\left(\pi(g)_{(2)} \pi(h)_{(2)} \otimes \pi(k)\right) \\
& =\gamma\left(\pi(h)_{(1)} \otimes \pi(k)_{(1)}\right) \gamma\left(\pi(g) \otimes \pi(h)_{(2)} \pi(k)_{(2)}\right),
\end{aligned}
$$

for all $g, h, k \in G$, and proceeding in a similar way one proves that $\tilde{\gamma}\left(h_{(1)} \otimes k_{(1)}\right) \widetilde{\gamma}\left(g \otimes h_{(2)} k_{(2)}\right)$ has the same expression. The convolution inverse of $\tilde{\gamma}$ is $\overline{\widetilde{\gamma}}(g \otimes h)=\bar{\gamma}(\pi(g) \otimes \pi(h))$ as easily proven by using again the fact that $\pi$ intertwines the coproducts.

We can deform the algebra product and antipode in the Hopf algebra $G$, and $H$, by using the 2-cocycles $\widetilde{\gamma}$ and $\gamma$ respectively. By Proposition 2.17 we obtain two new Hopf algebras which we denote by $G_{\tilde{\gamma}}$ and $H_{\gamma}$. Their algebra products are given respectively by

$$
g \cdot \tilde{\gamma} g^{\prime}=\gamma\left(\pi\left(g_{(1)}\right) \otimes \pi\left(g^{\prime}{ }_{(1)}\right)\right) g_{(2)} g_{(2)}^{\prime} \bar{\gamma}\left(\pi\left(g_{(3)}\right) \otimes \pi\left(g^{\prime}{ }_{(3)}\right)\right),
$$

for all $g, g^{\prime} \in G_{\widetilde{\gamma}}$, and

$$
h \cdot{ }_{\gamma} h^{\prime}=\gamma\left(h_{(1)} \otimes h_{(1)}^{\prime}\right) h_{(2)} h_{(2)}^{\prime} \bar{\gamma}\left(h_{(3)} \otimes h_{(3)}^{\prime}\right),
$$

for all $h, h^{\prime} \in H_{\gamma}$. The map $\pi: G \rightarrow H$ remains a Hopf algebra homomorphism with respect to the deformed Hopf algebra structures on $G_{\tilde{\gamma}}$ and $H_{\gamma}$ :

Lemma 4.2. The map

$$
\pi_{\gamma}: G_{\tilde{\gamma}} \longrightarrow H_{\gamma}, g \longmapsto \pi(g)
$$

is a surjective bialgebra homomorphism. 
Proof. Since the coproducts and counits of $G_{\widetilde{\gamma}}$ and $H_{\gamma}$ are not deformed by the twisting procedure it is clear that $\pi_{\gamma}$ is still a coalgebra map. We can easily check that $\pi_{\gamma}$ preserves also the deformed algebra product:

$$
\begin{aligned}
\pi_{\gamma}\left(g \cdot \tilde{\gamma} g^{\prime}\right) & =\gamma\left(\pi\left(g_{(1)}\right) \otimes \pi\left(g_{(1)}^{\prime}\right)\right) \pi\left(g_{(2)}\right) \pi\left(g_{(2)}^{\prime}\right) \bar{\gamma}\left(\pi\left(g_{(3)}\right) \otimes \pi\left(g_{(3)}^{\prime}\right)\right) \\
& =\gamma\left(\pi(g)_{(1)} \otimes \pi\left(g^{\prime}\right)_{(1)}\right) \pi\left(g_{(2)}\right) \pi\left(g_{(2)}^{\prime}\right) \bar{\gamma}\left(\pi(g)_{(3)} \otimes \pi\left(g^{\prime}\right)_{(3)}\right)=\pi_{\gamma}(g) \cdot \gamma \pi_{\gamma}\left(g^{\prime}\right),
\end{aligned}
$$

for all $g, g^{\prime} \in G_{\tilde{\gamma}}$.

It follows that the twisting procedure deforms the quantum homogeneous space $B=G^{c o H}$ into another quantum homogeneous space $B_{\widetilde{\gamma}}=G_{\widetilde{\gamma}}^{c o H_{\gamma}}$, which is isomorphic to $B$ only as a K-module but not in general as an algebra.

On the other hand, given a 2-cocycle $\gamma$ on $H$, we can deform $H$ into the Hopf algebra $H_{\gamma}$ as above, but consider $G$ simply as a right $H$-comodule algebra with coaction given in (4.1) and twist its algebra product accordingly to (2.28). In this way we get an $H_{\gamma}$-comodule algebra, $G_{\gamma}$, with product

$$
g \bullet \gamma g^{\prime}=g_{(1)} g_{(1)}^{\prime} \bar{\gamma}\left(\pi\left(g_{(2)}\right) \otimes \pi\left(g_{(2)}^{\prime}\right)\right),
$$

for all $g, g^{\prime} \in G_{\gamma}$. By Corollary 3.7, the extension $B=G_{\gamma}^{c o H_{\gamma}} \subseteq G_{\gamma}$ is an $H_{\gamma}$-Hopf-Galois extension if and only if the original extension $B \subseteq G$ was $H$-Hopf-Galois. However, as already remarked above, this twisted bundle has a total space which is just an algebra and the condition for $H_{\gamma}$ to be a quantum subgroup is lost, and so that of $B$ to be a quantum homogeneous space. To resolve this problem let us consider $K=H$ as an external Hopf algebra of symmetries coacting from the left on $G$. The Hopf algebra $G$ is also a left $H$ comodule algebra via

$$
\rho^{G}:=(\pi \otimes \mathrm{id}) \circ \Delta: G \longrightarrow H \otimes G, g \longmapsto \pi\left(g_{(1)}\right) \otimes g_{(2)} .
$$

Clearly, the left and right $H$-coactions $\rho^{G}$ and $\delta^{G}$ satisfy the compatibility condition (2.44), hence they structure $G$ as an $(H, H)$-bicomodule. Assume $B$ is a subcomodule for the left $H$-coaction. We can therefore twist the product in $G$ accordingly to Proposition 2.27(i) (with the special choice $\sigma=\gamma: H \otimes H \rightarrow \mathbb{K})$ in order to get an $\left(H_{\gamma}, H_{\gamma}\right)$-bicomodule algebra ${ }_{\gamma} G_{\gamma}$ with product

$$
g_{\gamma} \bullet_{\gamma} g^{\prime}=\gamma\left(\pi\left(g_{(1)}\right) \otimes \pi\left(g_{(1)}^{\prime}\right)\right) g_{(2)} g_{(2)}^{\prime} \bar{\gamma}\left(\pi\left(g_{(3)}\right) \otimes \pi\left(g_{(3)}^{\prime}\right)\right),
$$

for all $g, g^{\prime} \in{ }_{\gamma} G_{\gamma}$. Theorem 3.23 then implies that ${ }_{\gamma} B:={ }_{\gamma} G_{\gamma}^{c o H_{\gamma}} \subseteq{ }_{\gamma} G_{\gamma}$ is an $H_{\gamma}$-Hopf-Galois extension if and only if $B=G^{\mathrm{coH}} \subseteq G$ is a H-Hopf-Galois extension.

Proposition 4.3. The algebra ${ }_{\gamma} G_{\gamma}$ is isomorphic to the algebra underlying the Hopf algebra $G_{\tilde{\gamma}}$ and hence inherits from it a Hopf algebra structure. The subalgebra of coinvariants ${ }_{\gamma} B$ is isomorphic to the quantum homogeneous space $B_{\tilde{\gamma}}$.

Proof. By comparing (4.9) with (4.4) we have that the algebras ${ }_{\gamma} G_{\gamma}$ and $G_{\widetilde{\gamma}}$ are isomorphic via the identity map. For $b, b^{\prime} \in B_{\widetilde{\gamma}}$ we have

$$
b \cdot \tilde{\gamma} b^{\prime}=\gamma\left(\pi\left(b_{(1)}\right) \otimes \pi\left(b^{\prime}{ }_{(1)}\right)\right) b_{(2)} b_{(2)}^{\prime} \bar{\gamma}\left(\pi\left(b_{(3)}\right) \otimes \pi\left(b^{\prime}{ }_{(3)}\right)\right)=\gamma\left(\pi\left(b_{(1)}\right) \otimes \pi\left(b^{\prime}{ }_{(1)}\right)\right) b_{(2)} b_{(2)}^{\prime},
$$

because $B=B_{\widetilde{\gamma}}$ as $\mathbb{K}$-modules and hence $b, b^{\prime}$ are right $H$-coinvariant. Hence the result ${ }_{\gamma} B \simeq B_{\widetilde{\gamma}}$. 
As a direct consequence of Theorem 3.23 we then obtain that quantum principal bundles over quantum homogeneous spaces deform into quantum principal bundles over quantum homogeneous spaces:

Corollary 4.4. The extension $B_{\tilde{\gamma}} \subseteq G_{\tilde{\gamma}}$ of the quantum homogeneous space $B_{\tilde{\gamma}}$ is $H_{\gamma}$-Hopf-Galois if and only if the extension $B \subseteq G$ of the quantum homogeneous space B is H-Hopf-Galois.

\subsubsection{The quantum homogeneous spaces $S_{\theta}^{2 n}$ and their associated quantum principal bundles}

The $\theta$-spheres $S_{\theta}^{2 n}$ were introduced in [14] as noncommutative manifolds with the property that the Hochschild dimension equals the commutative dimension. They were shown to be homogeneous spaces of twisted deformations of $S O(2 n+1, \mathbb{R})$ in [35]. Their geometry was further studied in [13], see also [1]. We here revisit their explicit construction and as a corollary of the previous section conclude that the Hopf algebra of noncommutative coordinate functions $O\left(S O_{\theta}(2 n+1, \mathbb{R})\right)$ is a quantum principal bundle over the quantum homogeneous space $O\left(S_{\theta}^{2 n}\right)$ of noncommutative coordinate functions on the sphere. We then immediately conclude that the Hopf-Galois extension $O\left(S_{\theta}^{2 n}\right) \subset O\left(S O_{\theta}(2 n+1, \mathbb{R})\right)$ is a principal comodule algebra.

We begin by introducing the algebra of coordinate functions on $S O(2 n, \mathbb{R})$, on $S O(2 n+1, \mathbb{R})$ and on their quotient $S^{2 n}$. Let $O(M(2 n, \mathbb{R})), n \in \mathbb{N}$ be the commutative *-algebra over $\mathbb{C}$ with generators $a_{i j}, b_{i j}, a_{i j}^{*}=*\left(a_{i j}\right), b_{i j}^{*}=*\left(b_{i j}\right), i, j=1, \ldots n$. It is a bialgebra with coproduct and counit given in matrix notation as

$$
\Delta(M)=M \dot{\otimes} M \quad, \quad \varepsilon(M)=\mathbb{1}, \text { for } \quad M=\left(M_{I J}\right):=\left(\begin{array}{ll}
\left(a_{i j}\right) & \left(b_{i j}\right) \\
\left(b_{i j}^{*}\right) & \left(a_{i j}^{*}\right)
\end{array}\right),
$$

where $\dot{\otimes}$ denotes the combination of tensor product and matrix multiplication, $\mathbb{1}$ is the identity matrix and capital indices $I, J$ run from 1 to $2 n$. The Hopf algebra of coordinate functions on $S O(2 n, \mathbb{R})$ is the quotient $O(S O(2 n, \mathbb{R}))=O(M(2 n, \mathbb{R})) / I_{Q}$ where $I_{Q}$ is the bialgebra ideal defined by

$$
I_{Q}=\left\langle M^{t} Q M-Q ; M Q M^{t}-Q ; \operatorname{det}(M)-1\right\rangle, \quad Q:=\left(\begin{array}{cc}
0 & \mathbb{1}_{n} \\
\mathbb{1}_{n} & 0
\end{array}\right)=Q^{t}=Q^{-1} .
$$

In matrix notation the *-structure in $O(M(2 n, \mathbb{R}))$ is given by $*(M)=Q M Q$ so that $I_{Q}$ is easily seen to be a $*$-ideal. The $*$-bialgebra $O(S O(2 n, \mathbb{R}))$ is a $*$-Hopf algebra with antipode $S(M):=Q M^{t} Q^{-1}$. Notice that in $O(S O(2 n, \mathbb{R}))$ we have $M^{\dagger} M=\mathbb{1}=M M^{\dagger}$, where ${ }^{\dagger}$ indicates the composition of matrix transposition ${ }^{t}$ and $*$-conjugation.

Similarly, for the odd case let $O(M(2 n+1, \mathbb{R})), n \in \mathbb{N}$, be the commutative $*$-bialgebra with generators $a_{i j}, b_{i j}, a_{i j}^{*}=*\left(a_{i j}\right), b_{i j}^{*}=*\left(b_{i j}\right), u_{i}, v_{i}, u_{i}^{*}=*\left(u_{i}\right), v_{i}^{*}=*\left(v_{i}\right), i, j=1, \ldots n$, and $x=*(x)$. The coproduct and counit are given as

$$
\Delta(N)=N \dot{\otimes} N \quad, \quad \varepsilon(N)=\mathbb{1}, \text { where } N:=\left(\begin{array}{ccc}
\left(a_{i j}\right) & \left(b_{i j}\right) & \left(u_{i}\right) \\
\left(b_{i j}^{*}\right) & \left(a_{i j}^{*}\right) & \left(u_{i}^{*}\right) \\
\left(v_{i}\right) & \left(v_{i}^{*}\right) & x
\end{array}\right) .
$$


The algebra of coordinate functions on $S O(2 n+1, \mathbb{R})$ is the quotient $O(S O(2 n+1, \mathbb{R}))=$ $O(M(2 n+1, \mathbb{R})) / J_{Q}$ where $J_{Q}$ is the bialgebra $*$-ideal

$$
J_{Q}=\left\langle N^{t} Q N-Q ; N Q N^{t}-Q ; \operatorname{det}(N)-1\right\rangle, \quad Q:=\left(\begin{array}{ccc}
0 & \mathbb{1}_{n} & 0 \\
\mathbb{1}_{n} & 0 & 0 \\
0 & 0 & 1
\end{array}\right) .
$$

The *-structure can be written in terms of $Q$ as $*(N)=Q N Q^{-1}$. The *-bialgebra $O(S O(2 n+1, \mathbb{R}))$ is a *-Hopf algebra with antipode $S(N)=Q N^{t} Q=N^{\dagger}$.

The (commutative) Hopf algebra $O(S O(2 n, \mathbb{R}))$ is a quantum subgroup of $O(S O(2 n+1, \mathbb{R}))$ with surjective Hopf algebra morphism

$$
\pi: O(S O(2 n+1, \mathbb{R})) \longrightarrow O(S O(2 n, \mathbb{R})), \quad\left(\begin{array}{ccc}
\left(a_{i j}\right) & \left(b_{i j}\right) & \left(u_{i}\right) \\
\left(b_{i j}^{*}\right) & \left(a_{i j}^{*}\right) & \left(u_{i}^{*}\right) \\
\left(v_{i}\right) & \left(v_{i}^{*}\right) & x
\end{array}\right) \longmapsto\left(\begin{array}{ccc}
\left(a_{i j}\right) & \left(b_{i j}\right) & 0 \\
\left(b_{i j}^{*}\right) & \left(a_{i j}^{*}\right) & 0 \\
0 & 0 & 1
\end{array}\right)
$$

Hence there is a natural right coaction of $O(S O(2 n, \mathbb{R}))$ on $O(S O(2 n+1, \mathbb{R}))$, given by (cf. (4.1))

$$
\begin{aligned}
\delta:=(\mathrm{id} \otimes \pi) \Delta: O(S O(2 n+1, \mathbb{R})) & \longrightarrow O(S O(2 n+1, \mathbb{R})) \otimes O(S O(2 n, \mathbb{R})), \\
N & \longmapsto N \dot{\otimes} \pi(N) .
\end{aligned}
$$

The subalgebra $B \subset O(S O(2 n+1, \mathbb{R}))$ of coinvariants is generated by the elements in the last column of the defining matrix $N: u_{i}, u_{i}^{*}$ and $x$. It is isomorphic to the algebra of coordinate functions $O\left(S^{2 n}\right)$ on the even sphere $S^{2 n} \subset \mathbb{R}^{2 n+1}$, indeed from $N^{\dagger} N=\mathbb{1}$ we have that the generators of $B$ (rescaling the $u_{i}^{\prime}$ 's by $1 / \sqrt{2}$ ) satisfy the sphere equation $\sum_{i=1}^{n} u_{i}^{*} u_{i}+x^{2}=1$.

Finally, in this affine variety setting we can identify $O(S O(2 n+1, \mathbb{R}) \times S O(2 n, \mathbb{R}))$ with $O(S O(2 n+1, \mathbb{R})) \otimes O(S O(2 n, \mathbb{R}))$, and $O\left(S O(2 n+1, \mathbb{R}) \times{ }_{S^{2 n}} S O(2 n+1, \mathbb{R})\right)$ with $O(S O(2 n+$ $1, \mathbb{R})) \otimes_{O\left(S^{2 n}\right)} O(S O(2 n+1, \mathbb{R}))$, hence principality of the $S O(2 n, \mathbb{R})$-bundle $S O(2 n+1, \mathbb{R}) \rightarrow S^{2 n}$ implies that the algebra extension $O\left(S^{2 n}\right) \subset O(S O(2 n+1, \mathbb{R}))$ is Hopf Galois with $H=$ $O(S O(2 n, \mathbb{R}))$.

Next we consider a 2-cocycle $\gamma$ on the quantum subgroup $O(S O(2 n, \mathbb{R}))$, or rather on its maximal torus $\mathbb{T}^{n}$, and use it to deform the quantum homogeneous space $O\left(S^{2 n}\right)$ and the principal fibration on it. Let $O\left(\mathbb{T}^{n}\right)$ be the commutative *-algebra of functions on the $n$-torus with generators $t_{j}, t_{j}{ }^{*}=*\left(t_{j}\right)$ satisfying $t_{j} t_{j}{ }^{*}=1=t_{j}{ }^{*} t_{j}$ (no sum on $j$ ) for $j=1, \ldots n$. It is a Hopf algebra with

$$
\Delta(T)=T \dot{\otimes} T, \varepsilon(T)=\mathbb{1}, S(T)=T^{*}, \quad T:=\operatorname{diag}\left(t_{1}, \ldots t_{n}, t_{1}^{*}, \ldots t_{n}^{*}\right) .
$$

We consider the exponential 2-cocycle $\gamma$ on $O\left(\mathbb{T}^{n}\right)$ defined on the generators $t_{i}$ by

$$
\gamma\left(t_{j} \otimes t_{k}\right)=\exp \left(i \pi \theta_{j k}\right) ; \quad \theta_{j k}=-\theta_{k j} \in \mathbb{R}
$$

and extended to the whole algebra by requiring $\gamma(a b \otimes c)=\gamma\left(a \otimes c_{(1)}\right) \gamma\left(b \otimes c_{(2)}\right)$ and $\gamma(a \otimes b c)=$ $\gamma\left(a_{(1)} \otimes c\right) \gamma\left(a_{(2)} \otimes b\right)$, for all $a, b, c, \in O\left(\mathbb{T}^{n}\right)$, (cf. 3.31). The Hopf algebra $O\left(\mathbb{T}^{n}\right)$ is a quantum subgroup of $O(S O(2 n, \mathbb{R}))$ with projection

$$
M \mapsto T, \quad \text { i.e., } \quad a_{i j} \mapsto \delta_{j}^{i} t_{i} \quad ; \quad a_{i j}^{*} \mapsto \delta_{j}^{i} t_{i}^{*} \quad ; \quad b_{i j} \mapsto 0 \quad ; \quad b_{i j}^{*} \mapsto 0
$$


and hence the 2-cocycle $\gamma$ lifts by pullback to a 2-cocycle on $O(S O(2 n, \mathbb{R}))$ (see Lemma 4.1), that we still denote by $\gamma$. Now to deform with $\gamma$ the Hopf algebra $O(S O(2 n, \mathbb{R}))$ into the noncommutative Hopf algebra $O\left(S O_{\theta}(2 n, \mathbb{R})\right.$ ). The twisted algebra product is given by (cf. $(\underline{2.23})$

$$
M_{I J} \cdot \gamma M_{K L}=\gamma\left(T_{I} \otimes T_{K}\right) M_{I J} M_{K L} \bar{\gamma}\left(T_{J} \otimes T_{L}\right), \quad I, J, K, L=1, \ldots 2 n .
$$

Since $\gamma\left(T_{I} \otimes T_{K}\right)=\left(\gamma\left(T_{K} \otimes T_{I}\right)\right)^{-1}$ and similarly for $\bar{\gamma}$, it follows that the generators in $O\left(S O_{\theta}(2 n, \mathbb{R})\right)$ satisfy the commutation relations

$$
M_{I J} \cdot \gamma M_{K L}=\left(\gamma\left(T_{I} \otimes T_{K}\right)\right)^{2}\left(\bar{\gamma}\left(T_{J} \otimes T_{L}\right)\right)^{2} M_{K L} \cdot \gamma M_{I J}, \quad I, J, K, L=1, \ldots 2 n .
$$

Explicitly, setting $\lambda_{I J}:=\left(\gamma\left(T_{I} \otimes T_{J}\right)\right)^{2}$, so that $\lambda_{i j}=\exp \left(2 i \pi \theta_{i j}\right)$, and since $\bar{\gamma}\left(T_{J} \otimes T_{L}\right)=\gamma\left(T_{L} \otimes T_{J}\right)$, they read

$$
\begin{array}{ll}
a_{i j} \cdot \gamma a_{k l}=\lambda_{i k} \lambda_{l j} a_{k l} \cdot \gamma a_{i j} \quad, \quad a_{i j} \cdot \gamma b_{k l}^{*}=\lambda_{k i} \lambda_{l j} b_{k l}^{*} \cdot \gamma a_{i j} \\
a_{i j} \cdot \gamma b_{k l}=\lambda_{i k} \lambda_{j l} b_{k l} \cdot \gamma a_{i j}, & a_{i j} \cdot \gamma a_{k l}^{*}=\lambda_{k i} \lambda_{j l} a_{k l}^{*} \cdot \gamma a_{i j} \\
b_{i j} \cdot \gamma b_{k l}=\lambda_{i k} \lambda_{l j} b_{k l} \cdot \gamma b_{i j} \quad, & b_{i j} \cdot \gamma b_{k l}^{*}=\lambda_{k i} \lambda_{j l} b_{k l}^{*} \cdot \gamma \cdot b_{i j}
\end{array}
$$

together with their *-conjugated. It is also not difficult to show the equivalence of the quotient conditions (4.11) with the relations

$$
M^{t} \cdot \gamma Q \cdot \gamma M=Q, M \cdot \gamma, Q \cdot \gamma M^{t}=Q, \operatorname{det}_{\theta}(M)=1
$$

where the quantum determinant is defined by

$$
\operatorname{det}_{\theta}(M)=\sum_{\sigma \in \mathcal{P}_{2 n}}(-1)^{|\sigma|}\left(\prod_{\substack{I<J \\ \sigma_{I}>\sigma_{J}}} \lambda_{\sigma_{I} \sigma_{J}}\right) M_{1 \sigma_{1}} \cdot \gamma \ldots M_{2 n \sigma_{2 n}} .
$$

A quick way to prove the orthogonality relations is to observe that the new antipode, obtained according to Proposition 2.17 remains undeformed (sum on $L, K, R, P$ indices understood)

$$
\begin{aligned}
S_{\gamma}\left(M_{I J}\right) & =u_{\gamma}\left(M_{I L}\right) S\left(M_{L K}\right) \bar{u}_{\gamma}\left(M_{K J}\right)=\gamma\left(M_{I R} \otimes S\left(M_{R L}\right)\right) S\left(M_{L K}\right) \bar{\gamma}\left(S\left(M_{K P}\right) \otimes M_{P J}\right) \\
& =\gamma\left(T_{I} \otimes T_{I}\right) S\left(M_{I J}\right) \bar{\gamma}\left(T_{J} \otimes T_{J}\right)=S\left(M_{I J}\right),
\end{aligned}
$$

so that the orthogonality relations are the Hopf algebra relations $m_{\gamma} \circ\left(S_{\gamma} \otimes \mathrm{id}\right) \Delta(M)=\epsilon(M)$ and $m_{\gamma} \circ\left(\mathrm{id} \otimes S_{\gamma}\right) \Delta(M)=\epsilon(M)$. In order to obtain the quantum determinant relation first use $\gamma\left(T_{\sigma_{I}} \otimes T_{\sigma_{J}}\right)=\gamma\left(T_{\sigma_{J}} \otimes T_{\sigma_{I}}\right) \lambda_{\sigma_{I} \sigma_{J}}$ to show that for each permutation $\sigma$ we have the equality $\prod_{I<J, \sigma_{I}>\sigma_{I}} \lambda_{\sigma_{I} \sigma_{J}}=\prod_{I<J} \bar{\gamma}\left(T_{I} \otimes T_{J}\right) \gamma\left(T_{\sigma_{I}} \otimes T_{\sigma_{J}}\right)$. Next expand the twisted products in (4.19) in terms of the commutative products using $\gamma(a b \otimes c)=\gamma\left(a \otimes c_{(1)}\right) \gamma\left(b \otimes c_{(2)}\right)$ as well as the equivalent relation $\bar{\gamma}(a b \otimes c)=\bar{\gamma}\left(a \otimes c_{(2)}\right) \bar{\gamma}\left(b \otimes c_{(1)}\right)$ for all $a, b, c \in O(S O(2 n, \mathbb{R}))$ and notice that (4.19) becomes the usual determinant of $M$.

From (4.17) and (4.18) we see that the twisted Hopf algebra $O\left(S O_{\theta}(2 n, \mathbb{R})\right)$ can be described algebraically as the algebra over $\mathbb{C}$ freely generated by the matrix entries $M_{I J}$ modulo the ideal implementing the relations (4.18). The twisted Hopf algebras $O\left(S_{\theta}(n, \mathbb{R})\right)$ were studied in [3] (see also [31]) and in [13] as symmetries of $\theta$-planes and spheres.

We can lift the 2-cocycle from the quantum subgroup $O(S O(2 n, \mathbb{R}))$ to the Hopf algebra $O(S O(2 n+1, \mathbb{R}))$ by using the projection $\pi$ in (4.14) (or equivalently we can consider the torus 
$\mathbb{T}^{n}$ embedded in $\left.S O(2 n+1)\right)$. The resulting Hopf algebra is denoted by $O\left(S O_{\theta}(2 n+1, \mathbb{R})\right)$. It is the Hopf algebra over $\mathbb{C}$ freely generated by the matrix entries $N_{I J}$ modulo the relations

$$
N_{I J} \cdot \gamma N_{K L}=\left(\gamma\left(T_{I} \otimes T_{K}\right)\right)^{2}\left(\bar{\gamma}\left(T_{J} \otimes T_{L}\right)\right)^{2} N_{K L} \cdot \gamma N_{I J}, \quad I, J, K, L=1, \ldots 2 n+1,
$$

where now $T:=\operatorname{diag}\left(t_{1}, \ldots t_{n}, t_{1}^{*}, \ldots t_{n}^{*}, 1\right)$, and

$$
N^{t} \cdot{ }_{\gamma} Q \cdot{ }_{\gamma} N=Q, N \cdot{ }_{\gamma} Q \cdot{ }_{\gamma} N^{t}=Q, \operatorname{det}_{\theta}(N)=1,
$$

where $\operatorname{det}_{\theta}(N)$ is defined as in (4.19), just consider the permutation group $\mathcal{P}_{2 n+1}$.

As from Lemma 4.2 the quantum homogeneous space $B=O\left(S^{2 n}\right)$ is deformed into the quantum homogeneous space of coinvariants of $O\left(S O_{\theta}(2 n+1, \mathbb{R})\right)$ under the $O\left(S O_{\theta}(2 n, \mathbb{R})\right)$ coaction. This is the subalgebra $B_{\theta}=: O\left(S_{\theta}^{2 n}\right) \subset O\left(S O_{\theta}(2 n+1, \mathbb{R})\right)$ which is generated by the elements $u_{i}, u_{i}^{*}$ and $x$ entering the last column of the matrix $N$. Their commutation relations follow from (4.21)

$$
u_{i} \cdot \gamma u_{j}=\lambda_{i j} u_{j} \cdot \gamma u_{i} \quad ; \quad u_{i}^{*} \cdot \gamma u_{j}^{*}=\lambda_{i j} u_{j}^{*} \cdot \gamma u_{i}^{*} ; \quad u_{i} \cdot \gamma u_{j}^{*}=\lambda_{j i} u_{j}^{*} \cdot \gamma u_{i},
$$

while the orthogonality conditions (4.22) imply the sphere relation $\sum_{i=1}^{n} u_{i}^{*} \cdot \gamma u_{i}+x^{2}=1$. By Corollary 4.4 we conclude

Proposition 4.5. The algebra extension $O\left(S_{\theta}^{2 n}\right) \subset O\left(S O_{\theta}(2 n+1, \mathbb{R})\right)$ of the quantum homogeneous space $O\left(S_{\theta}^{2 n}\right)=O\left(S O_{\theta}(2 n+1, \mathbb{R})\right)^{\operatorname{coO}\left(S O_{\theta}(2 n, \mathbb{R})\right)}$ is Hopf-Galois.

Invertibility of the antipode and injectivity of $O\left(S O_{\theta}(2 n+1, \mathbb{R})\right)$ as an $O\left(S O_{\theta}(2 n, \mathbb{R})\right)$ comodule imply that $O\left(S_{\theta}^{2 n}\right) \subset O\left(S O_{\theta}(2 n+1, \mathbb{R})\right)$ is a principal comodule algebra.

\subsection{Twisting sheaves of Hopf-Galois extensions}

In classical geometry a principal bundle over a topological space $X$ can be given in terms of the local data of trivial product bundles over the open sets of a covering of $X$ and a set of transition functions which specify how to glue the local trivial pieces into a (possibly non trivial) global one. A local-type approach to noncommutative principal bundles was given in [30] by using sheaf theoretical methods. A quantum principle bundle consists in the data of two sheaves of $\mathbb{C}$-algebras over a (classical) topological space together with a quantum group, playing the role of the structure group, and a family of sheaf morphisms, satisfying some suitable conditions, as local trivializations. The two sheaves of algebras have to be regarded as the quantum analogues of the sheaves of functions over the base and total space of a classical fibration. The basic idea behind is that of considering a quantum space as a 'quantum ringed space' $\left(M, O_{M}\right)$, i.e. a topological space $M$ whose structure sheaf $O_{M}$ is a sheaf of (not necessarily commutative) algebras rather than of commutative rings.

A refinement of this sheaf theoretical approach to noncommutative bundles was proposed in [12] in terms of sheaves of Hopf-Galois extensions. For simplicity let us here assume all algebras are over a field.

Definition 4.6. Let $X$ be a topological space and $H$ a Hopf algebra. A sheaf $\mathscr{A}$ of (not necessarily commutative) algebras over $X$ is said to be a sheaf of $H$-Hopf-Galois extensions if: 
(i) $\mathscr{A}$ is a sheaf of (say) right $H$-comodules algebras: for each open $U \subseteq X, \mathscr{A}(U)$ is a right $H$-comodule algebra and for each open $W \subseteq U$ the restriction map $r_{U W}: \mathscr{A}(U) \rightarrow \mathscr{A}(W)$ is a morphism of $H$-comodule algebras;

(ii) for each open $U \subseteq X, \mathscr{A}(U)^{c o H} \subseteq \mathscr{A}(U)$ is a $H$-Hopf-Galois extension.

A sheaf $\mathscr{A}$ of $H$-Hopf-Galois extensions over a topological space $X$ is called locally cleft if there exists an open covering $\left\{U_{i}\right\}_{i \in I}$ of $X$ such that $\mathscr{A}\left(U_{i}\right)$ is cleft, $\forall i \in I$.

The sheaf $\mathscr{A}$ and its subsheaf $\mathscr{A}^{\mathrm{coH}}: U \mapsto \mathscr{A}(U)^{\mathrm{coH}}$ play the role of noncommutative analogues of the sheaf of functions on the total space, respectively base space, of the bundle. Notice that condition (ii) is equivalent to requiring just the algebra $\mathscr{A}(X)$ to be an $H$-HopfGalois extension, indeed it was observed in [12] that the property of being Hopf-Galois restricts locally: if on an open set $U$, the algebra extension $\mathscr{A}(U)^{c o H} \subseteq \mathscr{A}(U)$ is Hopf-Galois, then $\mathscr{A}(W)^{c o H} \subseteq \mathscr{A}(W)$ is a Hopf-Galois extension for any $W \subseteq U$. (This is the algebraic counterpart of the well-known classical fact that the restriction of a principal action is still principal).

The notions of quantum principal bundle introduced in [30] and that of locally cleft sheaf of Hopf-Galois extensions are closely related: every locally cleft sheaf of Hopf-Galois extensions is a quantum principal bundle in the sense of [30]. On the other hand, a sufficient condition for a quantum principal bundle in the sense of [30] to be a sheaf of Hopf-Galois extensions (in fact, locally cleft) is that the restriction maps are surjective (see [12, §4]).

Since a (locally cleft) sheaf $\mathscr{A}$ of $H$-Hopf-Galois extensions is in particular a sheaf of $H$-comodule algebras, given a 2-cocycle in $H$ we can apply the functor $\Gamma$ in (2.24) and obtain a new sheaf $\mathscr{A}_{\gamma}$ over the same topological space $X$. The sheaf $\mathscr{A}_{\gamma}$ is a sheaf of $H_{\gamma}$-comodule algebras and is defined by $\mathscr{A}_{\gamma}(U):=\Gamma(\mathscr{A}(U))=(\mathscr{A}(U))_{\gamma}$, with restriction maps given by morphisms of $H_{\gamma}$-comodule algebras $\Gamma\left(r_{U W}\right)=r_{U W}: \mathscr{A}_{\gamma}(U) \rightarrow \mathscr{A}_{\gamma}(W)$, for all $W \subset U$ open sets.

By Corollary 3.7 , we can conclude that $\mathscr{A}_{\gamma}$ is a (locally cleft) sheaf of $H_{\gamma}$-Hopf-Galois extensions if and only if $\mathscr{A}$ is a sheaf of (locally cleft) H-Hopf-Galois extensions. The subsheaves $\mathscr{A}_{\gamma}^{\mathrm{coH}_{\gamma}}$ and $\mathscr{A}^{\mathrm{coH}}$ over X coincide (i.e. they are isomorphic via the identity maps).

Let now $K$ be another Hopf algebra; we may assume the additional (restrictive) condition for the sheaf $\mathscr{A}$ to be valued in the category of $(K, H)$-bicomodule algebras, i.e. $\mathscr{A}(U) \in{ }^{K} \mathcal{A}^{H}$ for each open $U$ and the restriction maps are morphisms of $(K, H)$-bicomodule algebras. In this case we can deform $\mathscr{A}$ also by using a 2-cocycle $\sigma$ on the external Hopf algebra $K$, or even by using both $\sigma$ on $K$ and $\gamma$ on $H$. With the same reasoning as above, by using the results obtained in $\$ 3.2$ and $\$ 3.3$, the two sheaves $\sigma \mathscr{A}$ and $\sigma \mathscr{A} \gamma$ obtained in this way are sheaves of Hopf-Galois extensions if and only if the original sheaf $\mathscr{A}$ is. In general, the subsheaves ${ }_{\sigma} \mathscr{A}^{\mathrm{coH}}$ and ${ }_{\sigma} \mathscr{A}_{\gamma}^{\mathrm{CoH}_{\gamma}}$ of coinvariants will not coincide with $\mathscr{A}^{\mathrm{coH}}$. In the following subsection we provide an example.

\subsubsection{The Hopf bundle over $S_{\theta}^{4}$ as a twisted sheaf}

We describe the Hopf bundle over $S_{\theta}^{4}$ of Example 3.21 as a twist deformation of a sheaf of $(K, H)$-bicomodules algebras over the classical 4 -sphere $S^{4}$, where $H=O(S U(2))$ and 
$K=O\left(\mathbb{T}^{2}\right)$. The algebras ${ }_{\sigma} A=O\left(S_{\theta}^{7}\right)$ and ${ }_{\sigma} A^{c o H}=O\left(S_{\theta}^{4}\right)$ will be replaced by a locally cleft sheaf $_{\sigma} \mathscr{A}$ of $H$-Hopf-Galois extensions over $S^{4}$ and its subsheaf $\sigma \mathscr{A}^{c o H}$ of coinvariant elements.

We here outline a bottom up approach based on local transition functions on opens of $S^{4}$, a complementary top down approach starting from the total space $S^{7}$ description of the Hopf-Galois extension $O\left(S^{4}\right) \subseteq O\left(S^{7}\right)$ is presented in Appendix $\mathrm{C}$

As a first step we define the trivial Hopf-Galois extensions

$$
\begin{aligned}
& O\left(\alpha, \beta, x, c_{N}^{ \pm 1}\right) \subseteq O\left(\alpha, \beta, x, c_{N}^{ \pm 1}\right) \otimes H=: \mathscr{A}\left(U_{N}\right) \\
& O\left(\alpha, \beta, x, c_{S}^{ \pm 1}\right) \subseteq O\left(\alpha, \beta, x, c_{S}^{ \pm 1}\right) \otimes H=: \mathscr{A}\left(U_{S}\right) \\
& O\left(\alpha, \beta, x, c_{N}^{ \pm 1}, c_{S}^{ \pm 1}\right) \subseteq O\left(\alpha, \beta, x, c_{N}^{ \pm 1}, c_{S}^{ \pm 1}\right) \otimes H=: \mathscr{A}\left(U_{N S}\right)
\end{aligned}
$$

where $O\left(\alpha, \beta, x, c_{N}^{ \pm 1}\right)$ denotes the *-algebra generated by the $S^{4}$ coordinates $\alpha, \beta, x$ and by $c_{N}^{ \pm 1}$, with $c_{N}^{2}=\frac{1}{2}(1-x)$ (thus the generator $x$ becomes redundant). Since $x \neq 1$ (i.e., $c_{N} \neq 0$ ) except in the north pole $N$, these coordinates generate the algebra of coordinate functions on the open $U_{N}:=S^{4} \backslash\{N\} \simeq \mathbb{R}^{4}$. Similarly, the other coordinate algebras are over $U_{S}:=S^{4} \backslash\{S\}$, with $c_{S}^{2}=\frac{1}{2}(1+x)$, and $U_{N S}:=U_{N} \backslash\{S\}$ (cf. Appendix C.1).

Next we introduce the restriction maps defining the sheaf $\mathscr{A}$ of locally trivial Hopf-Galois extensions, and precisely the trivial restriction map

$$
r_{N, N S}^{\mathscr{A}}: O\left(\alpha, \beta, x, c_{N}^{ \pm 1}\right) \otimes H \stackrel{i_{N} \otimes \mathrm{id}}{\longrightarrow} O\left(\alpha, \beta, x, c_{N}^{ \pm 1}, c_{S}^{ \pm 1}\right) \otimes H
$$

(where $i_{N}$ denotes the canonical injection) and the nontrivial one (defined on the generators and extended as *-algebra map) 3

$$
\begin{aligned}
r_{S, N S}^{\mathscr{A}}: O\left(\alpha, \beta, x, c_{S}^{ \pm 1}\right) \otimes H & \longrightarrow O\left(\alpha, \beta, x, c_{N}^{ \pm 1}, c_{S}^{ \pm 1}\right) \otimes H, \\
1 \otimes\left(\begin{array}{cc}
w_{1} & -w_{2}^{*} \\
w_{2} & w_{1}^{*}
\end{array}\right) & \longmapsto \frac{1}{2} c_{N}^{-1} c_{S}^{-1}\left(\begin{array}{cc}
\alpha & -\beta^{*} \\
\beta & \alpha^{*}
\end{array}\right) \dot{\otimes}\left(\begin{array}{cc}
w_{1} & -w_{2}^{*} \\
w_{2} & w_{1}^{*}
\end{array}\right) \\
f \otimes 1 & \longmapsto i_{S}(f) \otimes 1
\end{aligned}
$$

where $i_{S}$ is the canonical injection $O\left(\alpha, \beta, x, c_{S}^{ \pm 1}\right) \stackrel{i_{S}}{\hookrightarrow} O\left(\alpha, \beta, x, c_{N}^{ \pm 1}, c_{S}^{ \pm 1}\right)$. It is straightforward to check that these restriction maps are morphisms of $H$-comodule algebras. Since $\left\{\emptyset, U_{N}, U_{S}, U_{N S}\right\}$ is a basis of the topology $\left\{\emptyset, U_{N}, U_{S}, U_{N S}, S^{4}\right\}$ the Hopf-Galois extensions in (4.23) and the restriction maps (4.24), (4.25) uniquely define the locally cleft sheaf $\mathscr{A}$ on the topology $\left\{\emptyset, U_{N}, U_{S}, U_{N S}, S^{4}\right\}$ (to $\emptyset$ we assign the one element algebra, terminal object in the category of algebras).

In particular the Hopf-Galois extension on the sphere $S^{4}$ is obtained as the pull-back (in the category of $*$-algebras)

$$
\mathscr{A}\left(S^{4}\right):=\left\{\left(a_{N}, a_{S}\right) \in \mathscr{A}\left(U_{N}\right) \times \mathscr{A}\left(U_{S}\right) \mid r_{N, N S}^{\mathscr{A}}\left(a_{N}\right)=r_{S, N S}^{\mathscr{A}}\left(a_{S}\right)\right\} .
$$

\footnotetext{
${ }^{3}$ This restriction map encodes the information on the transition function $g_{N S}$ characterizing the two charts $U_{N}, U_{S}$ description of the Hopf bundle $S^{7} \rightarrow S^{4}$. Indeed we have $g_{N S}: U_{N S} \rightarrow S U(2),(\alpha, \beta, x) \mapsto \frac{1}{2} c_{N}^{-1} c_{S}^{-1}\left(\begin{array}{cc}\alpha & -\beta^{*} \\ \beta & \alpha^{*}\end{array}\right)$, (we use the same notation for the coordinate functions and the point coordinates).
} 
From Lemma C.1 in Appendix C (for the notation used see (C.1) and (C.6)) and the Hcomodule algebra isomorphism (C.2) we immediately conclude that the pull-back $\mathscr{A}\left(S^{4}\right)$ is isomorphic to $O\left(S^{7}\right)$ as an $H$-comodule algebra. Then the subalgebra of coinvariants is $O\left(S^{4}\right)$ and the Hopf-Galois extension $\mathscr{A}\left(S^{4}\right)^{c o H} \subseteq \mathscr{A}\left(S^{4}\right)$ describes the instanton bundle $S^{7} \rightarrow S^{4}$.

Finally the sheaf $\mathscr{A}$ is a sheaf of $(K, H)$-bicomodule algebras, where the $K$-coactions are given by

$$
\begin{aligned}
\rho_{N}^{\mathscr{A}}: O\left(\alpha, \beta, x, c_{N}^{ \pm 1}\right) \otimes H & \longrightarrow K \otimes O\left(\alpha, \beta, x, c_{N}^{ \pm 1}\right) \otimes H, \\
1 \otimes\left(\begin{array}{cc}
w_{1} & -w_{2}^{*} \\
w_{2} & w_{1}^{*}
\end{array}\right) & \longmapsto\left(\begin{array}{cc}
t_{2} & 0 \\
0 & t_{2}^{*}
\end{array}\right) \dot{\otimes} \dot{\otimes}\left(\begin{array}{cc}
w_{1} & -w_{2}^{*} \\
w_{2} & w_{1}^{*}
\end{array}\right) \\
\alpha \otimes 1 & \longmapsto t_{1} t_{2}^{*} \otimes \alpha \otimes 1 \\
\beta \otimes 1 & \longmapsto t_{1}^{*} t_{2}^{*} \otimes \beta \otimes 1 \\
\rho_{S}^{\mathscr{A}}: O\left(\alpha, \beta, x, c_{S}^{ \pm 1}\right) \otimes H & \longrightarrow K \otimes O\left(\alpha, \beta, x, c_{S}^{ \pm 1}\right) \otimes H, \\
1 \otimes\left(\begin{array}{cc}
w_{1} & -w_{2}^{*} \\
w_{2} & w_{1}^{*}
\end{array}\right) & \longmapsto\left(\begin{array}{cc}
t_{1} & 0 \\
0 & t_{1}^{*}
\end{array}\right) \dot{\otimes} \dot{\otimes}\left(\begin{array}{cc}
w_{1} & -w_{2}^{*} \\
w_{2} & w_{1}^{*}
\end{array}\right) \\
\alpha \otimes 1 & \longmapsto t_{1} t_{2}^{*} \otimes \alpha \otimes 1 \\
\beta \otimes 1 & \longmapsto t_{1}^{*} t_{2}^{*} \otimes \beta \otimes 1
\end{aligned}
$$

with $x \otimes 1, c_{N}^{ \pm 1} \otimes 1$ and $c_{S}^{ \pm 1} \otimes 1$ coinvariant; likewise $\rho_{N S}^{\mathscr{A}}: O\left(\alpha, \beta, x, c_{N}^{ \pm 1}, c_{S}^{ \pm 1}\right) \otimes H \rightarrow K \otimes$ $O\left(\alpha, \beta, x, c_{N}^{ \pm 1}, c_{S}^{ \pm 1}\right) \otimes H$ is the extension of $\rho_{N}^{\mathscr{A}}$ obtained by defining $c_{S}^{ \pm 1} \otimes 1$ to be coinvariant. Observe that the K-coactions $\rho_{N}^{\mathscr{A}}$ and $\rho_{S}^{\mathscr{A}}$ differ from each other on $1 \otimes H$, henceforth the nontrivial restriction map $r_{S, N S}^{\mathscr{A}}$ is a morphism of $K$-comodule algebras.

We can now consider the 2-cocycle $\sigma$ in (3.31) on $K$ and use it to deform $\mathscr{A}\left(S^{4}\right)^{c o H} \subseteq \mathscr{A}\left(S^{4}\right)$ to $\sigma \mathscr{A}\left(S^{4}\right)^{\mathrm{coH}} \subseteq \sigma \mathscr{A}\left(S^{4}\right)$, and the commutative and trivial Hopf-Galois extensions in (4.23) into the noncommutative and trivial Hopf-Galois extensions

$$
\begin{aligned}
& { }_{\sigma} O\left(\alpha, \beta, x, c_{N}^{ \pm 1}\right) \subseteq{ }_{\sigma} O\left(\alpha, \beta, x, c_{N}^{ \pm 1}\right) \otimes H=:{ }_{\sigma} \mathscr{A}\left(U_{N}\right) \\
& { }_{\sigma} O\left(\alpha, \beta, x, c_{S}^{ \pm 1}\right) \subseteq{ }_{\sigma} O\left(\alpha, \beta, x, c_{S}^{ \pm 1}\right) \otimes H=:{ }_{\sigma} \mathscr{A}\left(U_{S}\right) \\
& { }_{\sigma} O\left(\alpha, \beta, x, c_{N}^{ \pm 1}, c_{S}^{ \pm 1}\right) \subseteq{ }_{\sigma} O\left(\alpha, \beta, x, c_{N}^{ \pm 1}, c_{S}^{ \pm 1}\right) \otimes H=:{ }_{\sigma} \mathscr{A}\left(U_{N S}\right) .
\end{aligned}
$$

The corresponding restriction maps are $\Sigma\left(r_{N, N S}^{\mathscr{A}}\right)=r_{N, N S}^{\mathscr{A}}: \sigma_{\sigma} \mathscr{A}\left(U_{N}\right) \rightarrow \sigma \mathscr{A}\left(U_{N S}\right), \Sigma\left(r_{S, N S}^{\mathscr{A}}\right)=r_{S, N S}^{\mathscr{A}}$ : $\sigma \mathscr{A}\left(U_{S}\right) \rightarrow \sigma \mathscr{A}\left(U_{N S}\right)$ and $\Sigma\left(p r_{1}^{\mathscr{A}}\right)=p r_{1}^{\mathscr{A}}: \sigma_{\sigma} \mathscr{A}\left(S^{4}\right) \rightarrow \sigma^{A}\left(U_{N}\right), \Sigma\left(p r_{2}^{\mathscr{A}}\right)=p r_{2}^{\mathscr{A}}:{ }_{\sigma} \mathscr{A}\left(S^{4}\right) \rightarrow$ $\sigma \mathscr{A}\left(U_{S}\right)$; they are $\left(K_{\sigma}, H\right)$-comodule maps and define the sheaf $\sigma \mathscr{A}$, that by construction is locally cleft. Since the Hopf-Galois extension $\mathscr{A}\left(S^{4}\right)^{c o H} \subseteq \mathscr{A}\left(S^{4}\right)$ is isomorphic to $O\left(S^{4}\right) \subseteq$ $O\left(S^{7}\right)$ then ${ }_{\sigma} \mathscr{A}\left(S^{4}\right)^{c o H} \subseteq \sigma_{\sigma}\left(S^{4}\right)$ is isomorphic to $O\left(S_{\theta}^{4}\right) \subseteq O\left(S_{\theta}^{7}\right)$, and the sheaf of Hopf Galois extensions ${ }_{\sigma} \mathscr{A}$ gives a sheaf description of the Hopf bundle over $S_{\theta}^{4}$ addressed in Example 3.21

\section{A Twists, 2-cocycles and untwisting}

We briefly outline the duality between the notions of Drinfeld twists [17, 18] and 2-cocycles that was mentioned in Section 2.2 and illustrate the 'untwisting procedure'. 


\section{A.1 Drinfeld twists}

Definition A.1. Let $\mathcal{U}$ be a bialgebra (or Hopf algebra). An invertible counital twist on $\mathcal{U}$, or simply a twist, is an invertible element $\mathcal{F} \in \mathcal{U} \otimes \mathcal{U}$ such that $(\varepsilon \otimes \mathrm{id})(\mathcal{F})=1=(\mathrm{id} \otimes \varepsilon)(\mathcal{F})$ and

$$
(\mathcal{F} \otimes 1)[(\Delta \otimes \mathrm{id})(\mathcal{F})]=(1 \otimes \mathcal{F})[(\mathrm{id} \otimes \Delta)(\mathcal{F})]
$$

We use the notations $\mathcal{F}=\mathrm{f}^{\alpha} \otimes \mathrm{f}_{\alpha} \in \mathcal{U} \otimes \mathcal{U}$ and $\mathcal{F}^{-1}=: \mathrm{f}^{-\alpha} \otimes \mathrm{f}_{-\alpha} \in \mathcal{U} \otimes \mathcal{U}$ (with summations understood).

Given a twist $\mathcal{F} \in \mathcal{U} \otimes \mathcal{U}$ we can deform the bialgebra (or Hopf algebra) $\mathcal{U}$ according to the following

Proposition A.2. Let $\mathcal{F}=f^{\alpha} \otimes f_{\alpha}$ be a twist on a bialgebra $\mathcal{U}$. Then the algebra $\mathcal{U}$ with coproduct

$$
\Delta_{\mathcal{F}}(\xi):=\mathcal{F} \Delta(\xi) \mathcal{F}^{-1}=f^{\alpha} \xi_{(1)} f^{-\beta} \otimes f_{\alpha} \xi_{(2)} f_{-\beta},
$$

for all $\xi \in \mathcal{U}$, and counit unchanged is a bialgebra, denoted $\mathcal{U}_{\mathcal{F}}$. If moreover $\mathcal{U}$ is a Hopf algebra, then the twisted bialgebra $\mathcal{U}_{\mathcal{F}}$ is a Hopf algebra with antipode $S_{\mathcal{F}}(\xi):=u_{\mathcal{F}} S(\xi) u_{\mathcal{F}}^{-1}$, where $u_{\mathcal{F}}:=f^{\alpha} S\left(f_{\alpha}\right)$, with inverse $u_{\mathcal{F}}^{-1}=S\left(f^{-\alpha}\right) f_{-\alpha}$.

Furthermore, if $A$ is a left $\mathcal{U}$-module algebra via $\triangleright: \mathcal{U} \otimes A \rightarrow A$, then the $\mathbb{K}$-module $A$ with unchanged unit and twisted product

$$
a \bullet_{\mathcal{F}} a^{\prime}:=\left(\mathrm{f}^{-\alpha} \triangleright a\right)\left(\mathrm{f}_{-\alpha} \triangleright a^{\prime}\right),
$$

for all $a, a^{\prime} \in A$, is a left $\mathcal{U}_{\mathcal{F}}$-module algebra with respect to the same action. We denote the twisted algebra by $A_{\mathcal{F}}$, with $\mathcal{U}_{\mathcal{F}}$-module structure given by $\triangleright$, now thought of as a map $\mathcal{U}_{\mathcal{F}} \otimes A_{\mathcal{F}} \rightarrow A_{\mathcal{F}}$.

\section{A.2 Duality between twists and 2-cocycles}

We here clarify how the two constructions of deforming by 2-cocycles and twists are dual to each other. Suppose $H$ and $\mathcal{U}$ are dually paired bialgebras (or Hopf algebras) with pairing $\langle\rangle:, \mathcal{U} \times H \rightarrow \mathbb{K}$, i.e., for all $\xi, \zeta \in \mathcal{U}$ and $h, k \in H$ we have $\langle\xi \zeta, h\rangle=\left\langle\xi, h_{(1)}\right\rangle\left\langle\zeta, h_{(2)}\right\rangle$, $\langle\xi, h k\rangle=\left\langle\xi_{(1)}, h\right\rangle\left\langle\xi_{(2)}, k\right\rangle,\left\langle\xi, 1_{H}\right\rangle=\varepsilon \mathcal{U}(\xi),\left\langle 1_{\mathcal{U}}, h\right\rangle=\varepsilon_{H}(h)$. Then to each invertible and counital twist $\mathcal{F}=\mathrm{f}^{\alpha} \otimes \mathrm{f}_{\alpha} \in \mathcal{U} \otimes \mathcal{U}$ there corresponds a convolution invertible and unital 2-cocycle $\gamma_{\mathcal{F}}: H \otimes H \rightarrow \mathbb{K}$ on $H$ defined by

$$
\gamma_{\mathcal{F}}(h \otimes k):=\left\langle\mathrm{f}^{\alpha}, h\right\rangle\left\langle\mathrm{f}_{\alpha}, k\right\rangle,
$$

for all $h, k \in H$, with convolution inverse $\bar{\gamma}_{\mathcal{F}}(h \otimes k)=\left\langle\mathrm{f}^{-\alpha}, h\right\rangle\left\langle\mathrm{f}_{-\alpha}, k\right\rangle$, for all $h, k \in H$. The 2-cocycle condition for $\gamma_{\mathcal{F}}$ follows from the twist condition for $\mathcal{F}$; indeed condition (A.1) in the $\mathcal{F}=\mathrm{f}^{\alpha} \otimes \mathrm{f}_{\alpha}$ notation reads as

$$
\mathbf{f}^{\alpha} \mathbf{f}^{\beta}{ }_{(1)} \otimes \mathbf{f}_{\alpha} \mathbf{f}_{(2)}^{\beta} \otimes \mathbf{f}_{\beta}=\mathbf{f}^{\alpha} \otimes \mathbf{f}^{\beta} \mathbf{f}_{\alpha(1)} \otimes \mathbf{f}_{\beta} \mathbf{f}_{\alpha(2)},
$$


so that

$$
\begin{aligned}
\gamma_{\mathcal{F}}\left(g_{(1)} \otimes h_{(1)}\right) \gamma_{\mathcal{F}}\left(g_{(2)} h_{(2)} \otimes k\right) & =\left\langle\mathrm{f}^{\alpha}, g_{(1)}\right\rangle\left\langle\mathrm{f}_{\alpha}, h_{(1)}\right\rangle\left\langle\mathrm{f}^{\beta}{ }_{(1)}, g_{(2)}\right\rangle\left\langle\mathrm{f}^{\beta}{ }_{(2)}, h_{(2)}\right\rangle\left\langle\mathrm{f}_{\beta}, k\right\rangle \\
& =\left\langle\mathrm{f}^{\alpha} \mathrm{f}^{\beta}{ }_{(1)}, g\right\rangle\left\langle\mathrm{f}_{\alpha} \mathrm{f}^{\beta}{ }_{(2)}, h\right\rangle\left\langle\mathrm{f}_{\beta}, k\right\rangle \\
& =\left\langle\mathrm{f}^{\alpha}, g\right\rangle\left\langle\mathrm{f}^{\beta} \mathbf{f}_{\alpha(1)}, h\right\rangle\left\langle\mathrm{f}_{\beta} \mathrm{f}_{\alpha(2)}, k\right\rangle \\
& =\left\langle\mathrm{f}^{\alpha}, g\right\rangle\left\langle\mathrm{f}^{\beta}, h_{(1)}\right\rangle\left\langle\mathrm{f}_{\alpha_{(1)},}, h_{(2)}\right\rangle\left\langle\mathrm{f}_{\beta}, k_{(1)}\right\rangle\left\langle\mathrm{f}_{\alpha(2)}, k_{(2)}\right\rangle \\
& =\left\langle\mathrm{f}^{\alpha}, g\right\rangle\left\langle\mathrm{f}^{\beta}, h_{(1)}\right\rangle\left\langle\mathrm{f}_{\beta}, k_{(1)}\right\rangle\left\langle\mathrm{f}_{\alpha}, h_{(2)} k_{(2)}\right\rangle \\
& =\gamma_{\mathcal{F}}\left(h_{(1)} \otimes k_{(1)}\right) \gamma_{\mathcal{F}}\left(g \otimes h_{(2)} k_{(2)}\right) .
\end{aligned}
$$

If we use $\mathcal{F}$ to twist the coproduct in $\mathcal{U}$ according to Proposition A.2 and $\gamma_{\mathcal{F}}$ to deform the product in $H$ as in Proposition 2.17 then the deformed bialgebras (or Hopf algebras) $\mathcal{U}_{\mathcal{F}}$ and $H_{\gamma_{\mathcal{F}}}$ are dually paired via the same pairing $\langle$,$\rangle ; indeed, it is easy to prove that$ $\left\langle\Delta_{\mathcal{F}}(\xi), h \otimes k\right\rangle=\left\langle\xi, h \cdot \gamma_{\mathcal{F}} k\right\rangle$ for all $\xi \in \mathcal{U}$ and $h, k \in H$.

Notice that if $A$ is a right $H$-comodule algebra via $\delta^{A}: A \rightarrow A \otimes H, a \mapsto a_{(0)} \otimes a_{(1)}$, then $A$ is a left $\mathcal{U}$-module algebra with left $\mathcal{U}$-action $\triangleright: \mathcal{U} \otimes A \rightarrow A,(\xi, a) \mapsto \xi \triangleright a:=a_{(0)}\left\langle\xi, a_{(1)}\right\rangle$. Hence, we can twist the product in $A$ by using $\mathcal{F}$ as in (A.3) or by using $\gamma_{\mathcal{F}}$ as in (2.28). The two constructions give the same algebra $A_{\mathcal{F}}=A_{\gamma_{\mathcal{F}}} ;$ indeed,

$$
\begin{aligned}
a \bullet_{\gamma_{\mathcal{F}}} a^{\prime} & =a_{(0)} a^{\prime}{ }_{(0)} \bar{\gamma}_{\mathcal{F}}\left(a_{(1)} \otimes a^{\prime}{ }_{(1)}\right)=a_{(0)} a^{\prime}{ }_{(0)}\left\langle\mathrm{f}^{-\alpha}, a_{(1)}\right\rangle\left\langle\mathrm{f}_{-\alpha}, a^{\prime}{ }_{(1)}\right\rangle \\
& =\left(\mathrm{f}^{-\alpha} \triangleright a\right)\left(\mathrm{f}_{-\alpha} \triangleright a^{\prime}\right)=a \bullet_{\mathcal{F}} a^{\prime},
\end{aligned}
$$

for all $a, a^{\prime} \in A$. Finally we observe that for a 2-cocycle $\gamma_{\mathcal{F}}$ associated with a twist $\mathcal{F}=$ $\mathrm{f}^{\alpha} \otimes \mathrm{f}_{\alpha} \in \mathcal{U} \otimes \mathcal{U}$, the map $\varphi_{A, A}$ introduced in Theorem 2.19 reads

$$
\varphi_{A, A}\left(a \otimes a^{\prime}\right)=\left(\mathrm{f}^{-\alpha} \triangleright a\right) \otimes\left(\mathrm{f}_{-\alpha} \triangleright a^{\prime}\right)=: \mathcal{F}^{-1} \triangleright\left(a \otimes a^{\prime}\right),
$$

for all $a, a^{\prime} \in A_{\gamma_{\mathcal{F}}}$.

\section{A.3 Untwisting with 2-cocycles}

We show that if we twist a bialgebra (or Hopf algebra) $H$ to $H_{\gamma}$ via a 2-cocycle $\gamma$ on $H$ we can untwist $H_{\gamma}$ to $H$ via the 2-cocycle $\bar{\gamma}$ on $H_{\gamma}$. More in general we have,

Proposition A.3. Let $\gamma$ be a 2-cocycle on a bialgebra (or Hopf algebra) $H$, and let $H_{\gamma}$ be the corresponding twisted bialgebra (or Hopf algebra). Then $\tau$ is a 2-cocycle on $H_{\gamma}$ if and only if $\tau * \gamma$ is a 2-cocycle on $H$. Furthermore, the twisted bialgebras (or Hopf algebras) $\left(H_{\gamma}\right)_{\tau}$ and $H_{\tau * \gamma}$ coincide.

Proof. By definition $\tau * \gamma$ is a 2-cocycle on $H$ if and only if for all $g, h, k \in H$,

$$
(\tau * \gamma)\left(g_{(1)} \otimes h_{(1)}\right)(\tau * \gamma)\left(g_{(2)} h_{(2)} \otimes k\right)=(\tau * \gamma)\left(h_{(1)} \otimes k_{(1)}\right)(\tau * \gamma)\left(g \otimes h_{(2)} k_{(2)}\right),
$$

this equality equivalently reads

$$
\begin{aligned}
\tau\left(g_{(1)} \otimes h_{(1)}\right) \gamma\left(g_{(2)} \otimes h_{(2)}\right) \tau\left(g_{(3)} h_{(3)} \otimes k_{(1)}\right) \gamma\left(g_{(4)} h_{(4)} \otimes k_{(2)}\right) \\
=\tau\left(h_{(1)} \otimes k_{(1)}\right) \gamma\left(h_{(2)} \otimes k_{(2)}\right) \tau\left(g_{(1)} \otimes h_{(3)} k_{(3)}\right) \gamma\left(g_{(2)} \otimes h_{(4)} k_{(4)}\right),
\end{aligned}
$$


and since $\gamma\left(g_{(1)} \otimes h_{(1)}\right) g_{(2)} h_{(2)}=g_{(1)} \cdot \gamma h_{(1)} \gamma\left(g_{(2)} \otimes h_{(2)}\right)$ for all $g, h$, the equality holds if and only if

$$
\begin{aligned}
\tau\left(g_{(1)} \otimes h_{(1)}\right) \tau\left(g_{(2)} \cdot \gamma h_{(2)} \otimes k\right) \gamma\left(g_{(3)} \otimes h_{(3)}\right) \gamma\left(g_{(4)} h_{(4)} \otimes k_{(2)}\right) \\
=\tau\left(h_{(1)} \otimes k_{(1)}\right) \tau\left(g \otimes h_{(2)} \cdot \gamma k_{(2)}\right) \gamma\left(h_{(3)} \otimes k_{(3)}\right) \gamma\left(g_{(2)} \otimes h_{(4)} k_{(4)}\right),
\end{aligned}
$$

i.e., since $\gamma$ is a twist on $H$, if and only if $\tau$ is a twist on $H_{\gamma}$ :

$$
\tau\left(g_{(1)} \otimes h_{(1)}\right) \tau\left(g_{(2)} \cdot \gamma h_{(2)} \otimes k\right)=\tau\left(h_{(1)} \otimes k_{(1)}\right) \tau\left(g \otimes h_{(2)} \cdot \gamma k_{(2)}\right) .
$$

It is straightforward to show that the twisted product ${ }_{\tau * \gamma}$ in $H_{\tau * \gamma}$ equals the twisted product $\gamma_{\tau}$ in $\left(H_{\gamma}\right)_{\tau}$; indeed,

$$
h \cdot \tau * \gamma=(\tau * \gamma)\left(h_{(1)} \otimes k_{(1)}\right) h_{(2)} k_{(2)}(\overline{\tau * \gamma})\left(h_{(3)} \otimes k_{(3)}\right)=\tau\left(h_{(1)} \otimes k_{(1)}\right) h_{(2)} \cdot \gamma k_{(2)} \tau\left(h_{(3)} \otimes k_{(3)}\right)=h \cdot \gamma_{\tau} k .
$$

Since the antipode if it exists is unique we immediately have the statement for Hopf algebras.

Setting $\tau=\bar{\gamma}$, since $\bar{\gamma} * \gamma=\varepsilon \otimes \varepsilon$ is trivially a 2-cocycle, we conclude that the twisted bialgebra (or Hopf algebra) $H_{\gamma}$ can be 'untwisted' via the convolution inverse $\bar{\gamma}$ :

Corollary A.4. If $\gamma$ is a 2-cocycle on the bialgebra (or Hopf algebra) $H$, then its convolution inverse $\bar{\gamma}$ is a 2-cocycle on the bialgebra (or Hopf algebra) $H_{\gamma}$, and $\left(H_{\gamma}\right)_{\bar{\gamma}}$ is isomorphic to $H$ (through the identity map).

\section{B Equivalence of closed monoidal categories and the (5-map}

In this section we show how the (5-map of Theorem 3.4 is related (by duality) to the natural transformation which establishes that twisting may be regarded as an equivalence of closed monoidal categories.

Recall from Theorem 3.4 that $65: H_{\gamma} \rightarrow \underline{H}_{\gamma}$ is a right $H_{\gamma}$-comodule isomorphism, where $H_{\gamma}$ carries the $\mathrm{Ad}_{\gamma}$-coaction and $\underline{H}_{\gamma}$ the Ad-coaction (regarded as an $H_{\gamma}$-coaction). Assume that $H^{\prime}$ and $H$ are dually paired Hopf algebras with pairing $\langle\rangle:, H^{\prime} \otimes H \rightarrow \mathbb{K}$, and let $\gamma$ be a 2-cocycle on $H$ with corresponding dual twist $\mathcal{F} \in H^{\prime} \otimes H^{\prime}$. Then the Hopf algebras $H_{\gamma}$ and $H_{\mathcal{F}}^{\prime}$ are dually paired and the right $H_{\gamma}$-comodules $\underline{H}_{\gamma}$ and $\underline{H}_{\gamma}$ are dually paired to the right

$H_{\mathcal{F}}^{\prime}$-modules $H_{\mathcal{F}}^{\prime}$ and $\underline{H}_{\mathcal{F}}^{\prime}$. These coincide with $H^{\prime}$ as $\overline{\mathbb{K}}$-modules and by definition have right $H_{\mathcal{F}}^{\prime}$-adjoint actions respectively given by

$$
\varangle_{\mathcal{F}}: \underline{H_{\mathcal{F}}^{\prime}} \otimes H_{\mathcal{F}}^{\prime} \longrightarrow \underline{H_{\mathcal{F}}^{\prime}}, \zeta \otimes \xi \longmapsto S_{\mathcal{F}}\left(\xi_{(1)_{\mathcal{F}}}\right) \zeta \xi_{(2)_{\mathcal{F}}}
$$

and

$$
\varangle: \underline{H}_{\mathcal{F}}^{\prime} \otimes H_{\mathcal{F}}^{\prime} \longrightarrow \underline{H}_{\mathcal{F}}^{\prime}, \zeta \otimes \xi \longmapsto S\left(\xi_{(1)}\right) \zeta \xi_{(2)} .
$$

(The dual pairing extends also to a dual pairing between right $H_{\gamma}$-comodule coalgebras and right $H_{\mathcal{F}}^{\prime}$-module algebras). 
The isomorphism $15: \underline{H}_{\gamma} \rightarrow \underline{H}_{\gamma}$ of Theorem 3.4 can be dualized to an isomorphism

$$
\mathfrak{6}^{\prime}: \underline{H}_{\mathcal{F}}^{\prime} \longrightarrow \underline{H_{\mathcal{F}}^{\prime}}
$$

by setting $\left\langle\mathfrak{G}^{\prime}(\xi), h\right\rangle=\left\langle\xi,(\mathfrak{\zeta}(h)\rangle\right.$, for all $\xi \in \underline{H}_{\mathcal{F}}^{\prime}$ and $h \in H_{\gamma}$. Explicitly, we have that

$$
\mathfrak{G}^{\prime}(\xi)=\mathbf{f}^{\beta}\left(\xi \triangleleft \mathrm{f}_{\beta}\right)=\mathrm{f}^{\beta} S\left(\mathrm{f}_{\beta_{(1)}}\right) \xi \mathrm{f}_{\left.\beta_{(2)}\right)}
$$

for all $\xi \in \underline{H}_{\mathcal{F}}^{\prime}$. Recall that right $H^{\prime}$-modules are equivalently left $H^{\prime o p c o p}$-modules and right $H_{\mathcal{F}}^{\prime}$-modules are left $\left(H_{\mathcal{F}}^{\prime}\right)^{\text {opcop }}=\left(H^{\prime o p c o p}\right)_{\mathcal{F} \text { opcop-modules, where }} \mathcal{F}^{\text {opcop }}=\mathcal{F}_{21}^{-1}=\mathrm{f}_{-\alpha} \otimes \mathfrak{f}^{-\alpha}$. The map $\mathfrak{5}^{\prime}: \underline{H}_{\mathcal{F}}^{\prime} \rightarrow H_{\mathcal{F}}^{\prime}$ is therefore equivalently an isomorphism of left $\left(H^{\prime \text { opcop }}\right)_{\mathcal{F} \text { opcop-modules, }}$ and using the left $\left(H^{\prime o p c o p}\right)_{\mathcal{F} \text { opcop-action it reads as }}$

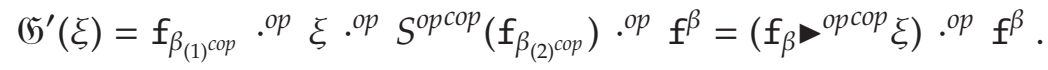

Referring to [4, Section 3.2], it follows that $\mathfrak{H}^{\prime}$ is precisely the isomorphism $D_{\mathcal{F} \text { opcop }}$ for the Hopf algebra $H^{\prime o p c o p}$ twisted by the twist $\mathcal{F}^{\text {opcop }}$. It has been shown in [5] that such $D$-maps have a categorical interpretation in terms of the natural isomorphism which establishes that twisting is an equivalence of closed monoidal categories. Hence, in conclusion, the dual of our $\mathfrak{5}$-map can be given a categorical interpretation.

\section{The twisted sheaf of the Hopf bundle over $S_{\theta}^{4}$ : top down ap- proach}

We complement the example of the twisted sheaf in \$4.2.1 by presenting a top down approach: we first describe $S^{7}$ as a ringed space, then on these algebras (rings) of coordinate functions on opens of $S^{7}$ we induce the $H$-coaction leading to a sheaf $\mathcal{A}$ of $H$-comodule algebras (with $H=O(S U(2)))$. Next we show that this is a locally cleft sheaf of $H$-Hopf Galois extensions, and as a corollary that it is naturally isomorphic to the sheaf $\mathscr{A}$ of $44.2 .1 \mathcal{A} \simeq \mathscr{A}$. Finally, in the last of the paragraphs titled in italics, the torus action on $\pi: S^{7} \rightarrow S^{4}$ is pulled back to this sheaf description and the corresponding twist deformation is obtained. In Section C.1 we study the subsheaf of $H$-coinvariants, it is generated by two copies (of the exponential version) of the Moyal-Weyl algebra on $\mathbb{R}_{\theta}^{4}$ that describe $S_{\theta}^{4}$ as a ringed space.

The sheaf $\mathcal{A}$ over $S^{4}$ of coordinate functions on opens of $S^{7}$

As in 84.2 .1 we consider the sphere $S^{4}$ with topology $\left\{\emptyset, U_{N}, U_{S}, U_{N S}, S^{4}\right\}$; it is generated by the basis with (open) sets $\emptyset, U_{N}, U_{S}$ and their intersection $U_{N S}$. The topology on $S^{4}$ induces a topology on $S^{7}$ given by the opens $\pi^{-1}(U)$, with $U$ open in $S^{4}$.

We define a sheaf $\mathcal{A}$ of algebras on $S^{4}$ by assigning an algebra to each open of the basis for the topology on $S^{4}$. To the empty set $\emptyset$ we assign the algebra $\mathcal{A}(\emptyset)$ that is the one-element algebra (where unit and zero elements coincide), while to the remaining open sets of the basis we define $\mathcal{A}(U)$ as quotients of central real extensions of $A=O\left(S^{7}\right)$, the coordinate algebra on $S^{7}$ generated by the commuting elements $z_{i}, z_{i}^{*}(i=1, \ldots 4)$ satisfying the sphere condition 
$\sum z_{i} z_{i}^{*}=1$. Explicitly we define

$$
\begin{aligned}
& \mathcal{A}\left(U_{N}\right):=O\left(S^{7}\right)\left[c_{N}, c_{N}^{-1}\right] /\left\langle z_{3} z_{3}^{*}+z_{4} z_{4}^{*}-c_{N}^{2}, c_{N} c_{N}^{-1}-1\right\rangle, \\
& \mathcal{A}\left(U_{S}\right):=O\left(S^{7}\right)\left[c_{S}, c_{S}^{-1}\right] /\left\langle z_{1} z_{1}^{*}+z_{2} z_{2}^{*}-c_{S}^{2}, c_{S} c_{S}^{-1}-1\right\rangle \\
& \mathcal{A}\left(U_{N S}\right):=O\left(S^{7}\right)\left[c_{N}^{ \pm 1}, c_{S}^{ \pm 1}\right] /\left\langle z_{3} z_{3}^{*}+z_{4} z_{4}^{*}-c_{N}^{2}, z_{1} z_{1}^{*}+z_{2} z_{2}^{*}-c_{S}^{2}, c_{N} c_{N}^{-1}-1, c_{S} c_{S}^{-1}-1\right\rangle .
\end{aligned}
$$

For each open set $U \subset S^{4}, \mathcal{A}(U)$ is the algebra of coordinate functions on $\pi^{-1}(U) \subset S^{7}$. Indeed extending the algebra $O\left(S^{7}\right)$ by the generator $c_{N}^{-1}$ corresponds, geometrically, to restricting to the subspace of $S^{7}$ of those points with $z_{3} z_{3}^{*}+z_{4} z_{4}^{*}$ never vanishing. Now recalling relation (3.30) between coordinates on $S^{7}$ and on $S^{4}$, we see that these are the points with $x \neq 1$, i.e., they are the points of $\pi^{-1}\left(U_{N}\right)$ (we use the same notation for the coordinate functions and the point coordinates). Conversely, enlarging the algebra with $c_{N}$ does not have a geometrical significance, but it is a pure algebraic operation designed to add the square root of the positive real element $z_{3} z_{3}^{*}+z_{4} z_{4}^{*}=c_{N}^{2}$ already belonging to the algebra $O\left(S^{7}\right)$. The same discussion is valid for the elements $c_{S}^{ \pm 1}$, so that $\mathcal{A}\left(U_{S}\right)$ and $\mathcal{A}\left(U_{N S}\right)$ are coordinate algebras on $\pi^{-1}\left(U_{S}\right)$ and $\pi^{-1}\left(U_{N S}\right)$ respectively.

The assignment $\mathcal{A}: U \mapsto \mathcal{A}(U)$, with restriction morphisms given by the canonical inclusions $i_{N}: \mathcal{A}\left(U_{N}\right) \hookrightarrow \mathcal{A}\left(U_{N S}\right)$ and $i_{S}: \mathcal{A}\left(U_{S}\right) \hookrightarrow \mathcal{A}\left(U_{N S}\right)$ defines 4 a sheaf of algebras over $S^{4}$. The algebra of global sections $\mathcal{A}\left(S^{4}\right)$ is the pull-back

$$
\mathcal{A}\left(S^{4}\right):=\left\{\left(a_{N}, a_{S}\right) \in \mathcal{A}\left(U_{N}\right) \times \mathcal{A}\left(U_{S}\right) \mid i_{N}\left(a_{N}\right)=i_{S}\left(a_{S}\right)\right\} \simeq O\left(S^{7}\right) .
$$

where $\times$ is the (categorical) product of *-algebras. In the last equality we have observed that $\mathcal{A}\left(S^{4}\right)$ is isomorphic to the coordinate algebra $O\left(S^{7}\right)$ of $S^{7}$, indeed $\mathcal{A}\left(S^{4}\right)$ is the diagonal of $O\left(S^{7}\right) \times O\left(S^{7}\right) \hookrightarrow \mathcal{A}\left(U_{N}\right) \times \mathcal{A}\left(U_{S}\right)$

The $H=O(S U(2))$-comodule structure on $\mathcal{A}$ and the subsheaf $\mathcal{B}=\mathcal{A}^{\mathrm{coH}}$

We recall from Example 3.21 that $\mathcal{A}\left(S^{4}\right)$ is a right $H=O(S U(2))$-comodule algebra with right coaction given by (cf. (3.28)):

$$
u \longmapsto u \dot{\otimes}\left(\begin{array}{cc}
w_{1} & -w_{2}^{*} \\
w_{2} & w_{1}^{*}
\end{array}\right) \quad, \quad u:=\left(\begin{array}{cccc}
z_{1} & z_{2} & z_{3} & z_{4} \\
-z_{2}^{*} & z_{1}^{*} & -z_{4}^{*} & z_{3}^{*}
\end{array}\right)^{t}
$$

(where $\dot{\otimes}$ denotes the composition of $\otimes$ with the matrix multiplication) and that the $H$ coinvariant subalgebra $B=A^{\mathrm{coH}}$ is generated by the elements

$$
\alpha:=2\left(z_{1} z_{3}^{*}+z_{2}^{*} z_{4}\right), \quad \beta:=2\left(z_{2} z_{3}^{*}-z_{1}^{*} z_{4}\right), \quad x:=z_{1} z_{1}^{*}+z_{2} z_{2}^{*}-z_{3} z_{3}^{*}-z_{4} z_{4}^{*},
$$

(and their *-conjugated $\alpha^{*}, \beta^{*}$, with $x^{*}=x$ ) that satisfy $\alpha^{*} \alpha+\beta^{*} \beta+x^{2}=1$. Thus the subalgebra $B=A^{c o H}$ of coinvariants is isomorphic to the algebra $O\left(S^{4}\right)$ of coordinate functions on $S^{4}$.

\footnotetext{
${ }^{4}$ We also have the restriction morphisms $\mathcal{A}(U) \rightarrow \mathcal{A}(\emptyset)$ that are canonical (and characterize the one-element algebra as the terminal object in the category of algebras).
} 
Since in particular the elements

$$
c_{N}^{2}=z_{3} z_{3}^{*}+z_{4} z_{4}^{*}=\frac{1}{2}(1-x), \quad c_{S}^{2}=z_{1} z_{1}^{*}+z_{2} z_{2}^{*}=\frac{1}{2}(1+x)
$$

are $H$-coinvariant, we then define, for each open set $U \subset S^{4}$, the $H$-comodule structure on $\mathcal{A}(U)$ via (C.3) and by requiring $c_{N}$ and $c_{S}$ to be $H$-coinvariant. In this way the canonical inclusions $\mathcal{A}\left(S^{4}\right) \hookrightarrow \mathcal{A}\left(U_{N}\right), \mathcal{A}\left(S^{4}\right) \hookrightarrow \mathcal{A}\left(U_{S}\right), \mathcal{A}\left(U_{N}\right) \stackrel{i_{N}}{\hookrightarrow} \mathcal{A}\left(U_{N S}\right)$ and $\mathcal{A}\left(U_{S}\right) \stackrel{i_{S}}{\hookrightarrow} \mathcal{A}\left(U_{N S}\right)$ are trivially right $H$-comodule algebra inclusions. We have thus shown that $\mathcal{A}$ is a sheaf of $H=O(S U(2))$-comodule algebras. The $H$-comodule structure on $\mathcal{A}\left(S^{4}\right)$ is obtained from the pull-back (C.2), thought now as pull-back of $H$-comodule algebras; the isomorphism $\mathcal{A}\left(S^{4}\right) \simeq O\left(S^{7}\right)$ then becomes an $H$-comodule algebra isomorphism.

The subalgebras of $H$-coinvariants are given by

$$
\mathcal{A}\left(U_{N}\right)^{c o H}=O\left(\alpha, \beta, x, c_{N}^{ \pm 1}\right) \quad, \quad \mathcal{A}\left(U_{S}\right)^{c o H}=O\left(\alpha, \beta, x, c_{S}^{ \pm 1}\right) \quad, \quad \mathcal{A}\left(U_{N S}\right)^{c o H}=O\left(\alpha, \beta, x, c_{N}^{ \pm 1}, c_{S}^{ \pm 1}\right)
$$

where $\mathcal{O}\left(\alpha, \beta, x, c_{N}^{ \pm 1}\right)$ denotes the *-subalgebra of $\mathcal{A}\left(U_{N}\right)$ generated by the elements $\alpha, \beta, x, c_{N}^{ \pm 1}$, and similarly for the other basic open sets. Notice that $\mathcal{A}^{\mathrm{coH}}(U)=\mathscr{A}^{\mathrm{coH}}(U)$ as defined in (4.23). The algebra of global coinvariant sections is $\mathcal{A}\left(S^{4}\right)^{c o H} \simeq O\left(S^{4}\right)$, and, similarly to (C.2), it is isomorphic to the pull-back

$$
\left\{\left(b_{N}, b_{S}\right) \in \mathcal{A}\left(U_{N}\right)^{c o H} \times \mathcal{A}\left(U_{S}\right)^{c o H} \mid i_{N}\left(b_{N}\right)=i_{S}\left(b_{S}\right)\right\} .
$$

In 8 C.1 we explicitly show that the subsheaf $\mathcal{A}^{c o H}$ of coinvariant elements (complemented by $\left.\mathcal{A}(\emptyset)^{c o H}=\mathcal{A}(\emptyset)\right)$ is that of coordinate functions on the opens $\emptyset, U_{N}, U_{S}, U_{N S}, S^{4}$.

The sheaf $\mathcal{A}$ is a locally cleft sheaf of $H-H o p f-G a l o i s ~ e x t e n s i o n s$

The $H$-comodule algebra isomorphism $\mathcal{A}\left(S^{4}\right) \simeq O\left(S^{7}\right)$ shows that the global sections $\mathcal{A}\left(S^{7}\right)$ are an $H=O(S U(2))$-Hopf-Galois extensions of the global coinvariant sections $\mathcal{A}\left(S^{4}\right)^{c o H} \simeq O\left(S^{4}\right)$. Recalling the general theory, cf. $\$ 4.2$ this shows that the sheaf $\mathcal{A}$ of $H$-comodule algebras is a sheaf of $H$-Hopf-Galois extensions.

In order to prove that $\mathcal{A}$ is locally cleft we consider the open covering $\left\{U_{N}, U_{S}\right\}$ of $S^{4}$ and show that $\mathcal{A}\left(U_{N}\right)^{c o H} \subseteq \mathcal{A}\left(U_{N}\right)$ and $\mathcal{A}\left(U_{S}\right)^{c 0 H} \subseteq \mathcal{A}\left(U_{S}\right)$ are cleft extensions.

We first observe that the matrix elements

$$
\left(\begin{array}{cc}
w_{1}^{N} & -\left(w_{2}^{N}\right)^{*} \\
w_{2}^{N} & \left(w_{1}^{N}\right)^{*}
\end{array}\right):=c_{N}^{-1}\left(\begin{array}{cc}
z_{3} & -z_{4}^{*} \\
z_{4} & z_{3}^{*}
\end{array}\right)
$$

generate a *-subalgebra of $\mathcal{A}\left(U_{N}\right)$ isomorphic to $H=O(S U(2))$; indeed this matrix has unit determinant since $c_{N}^{-2}\left(z_{3} z_{3}^{*}+z_{4} z_{4}^{*}\right)=1$. Similarly the matrix elements

$$
\left(\begin{array}{cc}
w_{1}^{S} & -\left(w_{2}^{S}\right)^{*} \\
w_{2}^{S} & \left(w_{1}^{S}\right)^{*}
\end{array}\right):=c_{S}^{-1}\left(\begin{array}{cc}
z_{1} & -z_{2}^{*} \\
z_{2} & z_{1}^{*}
\end{array}\right),
$$

generate an $H=O(S U(2)) *$-subalgebra of $\mathcal{A}\left(U_{N}\right)$. 
By using the matrix $u$ in (C.3) and the matrix projector $\mathrm{P}=u u^{*}$ (whose entries are the generators of $S^{4}$, see (3.29) ) we introduce "local trivialization maps": 5

$$
\begin{aligned}
& \Psi_{N}: \mathcal{A}\left(U_{N}\right) \longrightarrow \mathcal{A}\left(U_{N}\right)^{c o H} \otimes H \\
& \Psi_{S}: \mathcal{A}\left(U_{S}\right) \longrightarrow \mathcal{A}\left(U_{S}\right)^{c o H} \otimes H \\
& u \longmapsto c_{N}^{-1} \mathrm{P} \dot{\otimes} c_{N}^{-1}\left(\begin{array}{cc}
1 & 0 \\
0 & 1 \\
z_{3} & -z_{4}^{*} \\
z_{4} & z_{3}^{*}
\end{array}\right), \\
& u \longmapsto c_{S}^{-1} \mathrm{P} \dot{\otimes} c_{S}^{-1}\left(\begin{array}{cc}
z_{1} & -z_{2}^{*} \\
z_{2} & z_{1}^{*} \\
1 & 0 \\
0 & 1
\end{array}\right),
\end{aligned}
$$

(extended as *-algebra maps).

Lemma C.1. The maps $\Psi_{N}$ and $\Psi_{S}$ in (C.10) are well defined algebra morphisms and are isomorphisms of left $\mathcal{A}\left(U_{N}\right)^{c o H}$-modules (respectively $\mathcal{A}\left(U_{S}\right)^{c o H}$-modules) and also of right $H$-comodule algebras, where $\mathcal{A}\left(U_{N}\right)^{c o H} \otimes H_{N}$ and $\mathcal{A}\left(U_{S}\right)^{c o H} \otimes H_{S}$ have H-coaction given by the coproduct, id $\otimes \Delta$ (cf. (2.3). Hence they structure $\mathcal{A}\left(U_{N}\right)$ and $\mathcal{A}\left(U_{S}\right)$ as cleft Hopf-Galois extension.

Proof. Explicitly, these local trivialization maps are given by

$$
\begin{aligned}
\Psi_{N}: \mathcal{A}\left(U_{N}\right) & \longrightarrow \mathcal{A}\left(U_{N}\right)^{c H} \otimes H \\
z_{1} & \longmapsto z_{1}^{N}:=\frac{c_{N}^{-1}}{2}\left(\alpha \otimes w_{1}^{N}-\beta^{*} \otimes w_{2}^{N}\right) \\
z_{2} & \longmapsto z_{2}^{N}:=\frac{c_{N}^{-1}}{2}\left(\beta \otimes w_{1}^{N}+\alpha^{*} \otimes w_{2}^{N}\right) \\
z_{3} & \longmapsto z_{3}^{N}:=c_{N} \otimes w_{1}^{N} \\
z_{4} & \longmapsto z_{4}^{N}:=c_{N} \otimes w_{2}^{N} \\
c_{N}^{ \pm 1} & \longmapsto c_{N}^{ \pm 1} \otimes\left|w^{N}\right|^{2}=c_{N}^{ \pm 1} \otimes 1,
\end{aligned}
$$

\footnotetext{
${ }^{5}$ Recall that the Hopf bundle $\pi: S^{7} \rightarrow S^{4}$ trivializes on the two charts $U_{N}, U_{S}$ with trivializations (we use the same notation for the coordinate functions and the point coordinates)

$$
\pi^{-1}\left(U_{N}\right) \longrightarrow U_{N} \times S U(2), z=\left(z_{1}, z_{2}, z_{3}, z_{4}\right) \longmapsto\left(\pi(z), \frac{1}{\left(\left|z_{3}\right|^{2}+\left|z_{4}\right|^{2}\right)^{\frac{1}{2}}}\left(\begin{array}{cc}
z_{3} & -z_{4}^{*} \\
z_{4} & z_{3}^{*}
\end{array}\right)\right)
$$

and

$$
\pi^{-1}\left(U_{S}\right) \longrightarrow U_{S} \times S U(2), \quad z=\left(z_{1}, z_{2}, z_{3}, z_{4}\right) \longmapsto\left(\pi(z), \frac{1}{\left(\left|z_{1}\right|^{2}+\left|z_{2}\right|^{2}\right)^{\frac{1}{2}}}\left(\begin{array}{cc}
z_{1} & -z_{2}^{*} \\
z_{2} & z_{1}^{*}
\end{array}\right)\right) .
$$

The datum of the transition functions characterizing the bundle is contained in the trivialization maps. In the present case we have just one intersection $U_{N S}$, defining the transition function

$$
g_{N S}: U_{N S} \longrightarrow S U(2), \quad \pi(z) \longmapsto \frac{\left(\left|z_{3}\right|^{2}+\left|z_{4}\right|^{2}\right)^{\frac{1}{2}}}{\left(\left|z_{1}\right|^{2}+\left|z_{2}\right|^{2}\right)^{\frac{1}{2}}}\left(\begin{array}{cc}
z_{1} & -z_{2}^{*} \\
z_{2} & z_{1}^{*}
\end{array}\right)\left(\begin{array}{cc}
z_{3} & -z_{4}^{*} \\
z_{4} & z_{3}^{*}
\end{array}\right)^{-1},
$$
}

i.e.

$$
(\alpha, \beta, x) \longmapsto \frac{1}{2} c_{N}^{-1} c_{S}^{-1}\left(\begin{array}{cc}
\alpha & -\beta^{*} \\
\beta & \alpha^{*}
\end{array}\right)
$$


and

$$
\begin{aligned}
\Psi_{S}: \mathcal{A}\left(U_{S}\right) & \longrightarrow \mathcal{A}\left(U_{S}\right)^{c o H} \otimes H \\
z_{1} & \longmapsto z_{1}^{S}:=c_{S} \otimes w_{1}^{S} \\
z_{2} & \longmapsto z_{2}^{S}:=c_{S} \otimes w_{2}^{S} \\
z_{3} & \longmapsto z_{3}^{S}:=\frac{c_{S}^{-1}}{2}\left(\alpha^{*} \otimes w_{1}^{S}+\beta^{*} \otimes w_{2}^{S}\right) \\
z_{4} & \longmapsto z_{4}^{S}:=\frac{c_{S}^{-1}}{2}\left(-\beta \otimes w_{1}^{S}+\alpha \otimes w_{2}^{S}\right) \\
c_{S}^{ \pm 1} & \longmapsto c_{S}^{ \pm 1} \otimes\left|w^{S}\right|^{2}=c_{S}^{ \pm 1} \otimes 1 .
\end{aligned}
$$

Since

$$
\begin{aligned}
& z_{1}^{N}\left(z_{3}^{N}\right)^{*}+\left(z_{2}^{N}\right)^{*} z_{4}^{N}=\frac{1}{2} \alpha \otimes 1, \quad z_{2}^{N}\left(z_{3}^{N}\right)^{*}-\left(z_{1}^{N}\right)^{*} z_{4}^{N}=\frac{1}{2} \beta \otimes 1, \\
& z_{1}^{N}\left(z_{1}^{N}\right)^{*}+z_{2}^{N}\left(z_{2}^{N}\right)^{*}-z_{3}^{N}\left(z_{3}^{N}\right)^{*}-z_{4}^{N}\left(z_{4}^{N}\right)^{*}=\left(1-2 c_{N}^{2}\right)=x \otimes 1,
\end{aligned}
$$

the elements $z_{i}^{N}, c_{N}^{ \pm}$generate $\mathcal{A}\left(U_{N}\right)^{c o H} \otimes H$. Use of (C.5) shows that $\Psi_{N}\left(\sum z_{i} z_{i}^{*}\right)=\sum z_{i}^{N}\left(z_{i}^{N}\right)^{*}=1$, and $\Psi_{N}\left(z_{3} z_{3}^{*}+z_{4} z_{4}^{*}\right)=z_{3}^{N}\left(z_{3}^{N}\right)^{*}+z_{4}^{N}\left(z_{4}^{N}\right)^{*}=c_{N}^{2}$ so that $\Psi_{N}$ is a well defined algebra map. It is a one to one correspondence between generators and relations defining $\mathcal{A}\left(U_{N}\right)$ and generators and relations defining $\mathcal{A}\left(U_{N}\right)^{c o H} \otimes H$, hence it is a *-algebra isomorphism. Identical expressions hold for the elements $z_{i}^{S}, c_{S}^{ \pm 1}$, so that also $\Psi_{S}$ is a $*$-algebra isomorphism. It is evident from (C.3) that $\Psi_{N}$ and $\Psi_{S}$ are $H$-comodule maps and left $\mathcal{A}\left(U_{N}\right)^{c o H}$, respectively $\mathcal{A}\left(U_{S}\right)^{c 0 H}$-module maps.

Notice that as a corollary of this lemma the sheaf $\mathcal{A}$ and the sheaf $\mathscr{A}$ of $\$ 4.2 .1$ are naturally isomorphic sheaves of $H$-Hopf-Galois extensions on $S^{4}$.

The K-comodule structure on $\mathcal{A}$ and the locally cleft sheaf of $H$-Hopf-Galois extensions ${ }_{\sigma} \mathcal{A}$

Recall from Example 3.21 that $K=O\left(\mathbb{T}^{2}\right)$ denotes the commutative *-Hopf algebra of coordinates on the torus $\mathbb{T}^{2}$. For each open set $U$, the algebra $\mathcal{A}(U)$ carries a $K$-comodule structure where the left $K$-coaction is the *-algebra map defined on generators by (cf. (3.32))

$$
u \longmapsto \operatorname{diag}\left(t_{1}, t_{1}^{*}, t_{2}, t_{2}^{*}\right) \dot{\otimes} u \quad, \quad c_{N}^{ \pm 1} \longmapsto 1 \otimes c_{N}^{ \pm 1} \quad, \quad c_{S}^{ \pm 1} \longmapsto 1 \otimes c_{S}^{ \pm 1}
$$

(here $\dot{\otimes}$ denotes the composition of $\otimes$ with the matrix multiplication). For each open $U$ the $K$-coaction and the $H$-coaction given in (C.3) satisfy the compatibility condition (2.44) and thus $\mathcal{A}(U)$ is a $(K, H)$-bicomodule algebra. Furthermore the restriction morphisms of $\mathcal{A}$ are morphisms of $(K, H)$-bicomodule algebras, so that $\mathcal{A}$ is a sheaf of $(K, H)$-bicomodule algebras.

We can now consider the 2-cocycle $\sigma$ in (3.31) on $K$ and use it to deform the sheaf $\mathcal{A}$. According to the general theory in $\$ 4.2$, for each open set $U \subseteq S^{4}, \mathcal{A}(U)$ is deformed into a $\left(K_{\sigma}, H\right)$-bicomodule algebra ${ }_{\sigma} \mathcal{A}(U)$ that is also an $H$-Hopf-Galois extension. The resulting sheaf of $H$-Hopf-Galois extensions ${ }_{\sigma} \mathcal{A}$ gives a sheaf-description of the Hopf bundle over $S_{\theta}^{4}$ addressed in Example 3.21 because, since $\mathcal{A}\left(S^{4}\right) \simeq O\left(S^{7}\right)$ and $\mathcal{A}\left(S^{4}\right)^{c 0 H} \simeq O\left(S^{4}\right)$ (cf. discussion after (C.2) and before (C.7)), then ${ }_{\sigma} \mathcal{H}\left(S^{4}\right) \simeq O\left(S_{\theta}^{7}\right)$ and ${ }_{\sigma} \mathcal{A}\left(S^{4}\right)^{c o H} \simeq O\left(S_{\theta}^{4}\right)$. 


\section{C.1 The spheres $S^{4}$ and $S_{\theta}^{4}$ as ringed spaces}

We show that the subsheaf of coinvariant elements $\mathscr{A}^{\mathrm{coH}}=\mathcal{A}^{\mathrm{coH}}$ introduced in $\$ 4.2 .1$ (see also (C.6)) is that of the algebras (rings) of coordinate functions on the opens $U_{N}, U_{S}, U_{N S}, S^{4}$, and correspondingly, that the subsheaf of coinvariant elements $\sigma_{\sigma} \mathscr{A}^{\mathrm{coH}}$ arises from the Moyal-Weyl algebra on $\mathbb{R}_{\theta}^{4}$.

Lemma C.2. Let $\mathscr{B}\left(U_{N}\right)$ denote the commutative *-algebra generated by elements $x_{1}, x_{2}, x_{1}^{*}, x_{2}^{*}$ together with $\rho_{N}^{ \pm 1}$ satisfying $\rho_{N}^{-2}\left(1+x_{1} x_{1}^{*}+x_{2} x_{2}^{*}\right)=1$ and $\rho_{N}^{-1} \rho_{N}=1$. Let $\mathscr{B}\left(U_{S}\right)$ denote the commutative *-algebra generated by elements $y_{1}, y_{2}, y_{1}^{*}, y_{2}^{*}$ together with $\rho_{s}^{ \pm 1}$ satisfying $\rho_{s}^{-2}\left(1+y_{1} y_{1}^{*}+y_{2} y_{2}^{*}\right)=1$ and $\rho_{s}^{-1} \rho_{s}=1$. The maps

$$
\begin{aligned}
& \phi_{N}: \mathscr{B}\left(U_{N}\right) \longrightarrow \mathscr{A}\left(U_{N}\right)^{c o H} \quad \phi_{S}: \mathscr{B}\left(U_{S}\right) \longrightarrow \mathscr{A}\left(U_{S}\right)^{c o H} \\
& x_{1} \longmapsto \frac{1}{2} \alpha c_{N}^{-2} \quad y_{1} \longmapsto \frac{1}{2} \alpha c_{S}^{-2} \\
& x_{2} \longmapsto \frac{1}{2} \beta c_{N}^{-2} \quad y_{2} \longmapsto \frac{1}{2} \beta c_{S}^{-2} \\
& \rho_{N}^{ \pm 1} \longmapsto c_{N}^{\mp 1} \quad \rho_{S}^{ \pm 1} \longmapsto c_{S}^{\mp 1}
\end{aligned}
$$

(extended as *-algebra maps) define *-algebra isomorphisms.

Proof. The inverse maps are given by

$$
\begin{array}{ll}
\phi_{N}^{-1}: & c_{N}^{\mp 1} \longmapsto \rho_{N}^{ \pm 1} ; \quad \alpha \longmapsto 2 x_{1} \rho_{N}^{-2} ; \quad \beta \longmapsto 2 x_{2} \rho_{N}^{-2} ; \\
\phi_{S}^{-1}: \quad c_{S}^{\mp 1} \longmapsto \rho_{S}^{ \pm 1} ; \quad \alpha \longmapsto 2 y_{1} \rho_{S}^{-2} ; \quad \beta \longmapsto 2 y_{2} \rho_{S}^{-2} .
\end{array}
$$

By using $(1-x)=2 c_{N}^{2}$, valid in $\mathscr{A}\left(U_{N}\right)^{c o H}$, it is easy to show that the map $\phi_{N}$ is an algebra map, i.e. preserves the identity $\rho_{N}^{-2}\left(1+x_{1} x_{1}^{*}+x_{2} x_{2}^{*}\right)=1$. An analogous computation, using again (C.5), shows that $\phi_{s}$ is an algebra map.

Because of this lemma the algebras $\mathscr{A}\left(U_{N}\right)^{c o H}$ and $\mathscr{A}\left(U_{S}\right)^{c o H}$ are interpreted as two (isomorphic) copies of the algebra of coordinate functions on $\mathbb{R}^{4}$. (Adding to the algebra generated by $x_{1}, x_{2}, x_{1}^{*}, x_{2}^{*}$ the generators $\rho_{N}^{ \pm 1}$ is geometrically ineffective, similarly for $\rho_{S}^{ \pm 1}$ ). Specifically, they describe the algebras of coordinate functions on the open sets $U_{N}, U_{S}$, obtained via stereographic projections from the North and South poles of the 4-sphere 6

Similarly, the following lemma shows that the algebra $\mathscr{A}\left(U_{N S}\right)^{c O H}$ is that of coordinate functions on $\mathbb{R}^{4}$ minus the origin.

Lemma C.3. We denote by $\mathscr{B}\left(U_{N S}\right)$ the algebra extension of $\mathscr{B}\left(U_{N}\right)$ by central real elements $r^{ \pm 1}$, satisfying $r r^{-1}=1, r^{2}:=x_{1} x_{1}^{*}+x_{2} x_{2}^{*}$. The map $\phi_{N}$ in (C.15) extends to an algebra isomorphism $\phi_{\mathrm{NS}}: \mathscr{B}\left(U_{N S}\right) \stackrel{\simeq}{\rightarrow} \mathscr{A}\left(U_{N S}\right)^{c o H}$ by setting

$$
r^{-1} \longmapsto c_{N} c_{S}^{-1} \quad, \quad r \longmapsto c_{S} c_{N}^{-1}
$$

\footnotetext{
${ }^{6}$ Using the same notation for the coordinate functions and the point coordinates, a point $\left(\alpha, \alpha^{*}, \beta, \beta^{*}, x\right) \in S^{4}$ maps via the stereographic projection from the North pole to the point $\left(x_{1}, x_{1}^{*}, x_{2}, x_{2}^{*}\right) \in \mathbb{R}^{4}$ with coordinates $x_{1}=\alpha(1-x)^{-1}, x_{2}=\beta(1-x)^{-1}$. While, via stereographic projection from the South pole, it projects to the point with coordinates $y_{1}=\alpha(1+x)^{-1}, y_{2}=\beta(1+x)^{-1}$. The coordinate function $\rho_{N}^{-1}$ in $\mathscr{B}\left(U_{N}\right)$, as $\rho_{S}^{-1}$ in $\mathscr{B}\left(U_{S}\right)$, has no geometrical significance since $1+x_{1} x_{1}^{*}+x_{2} x_{2}^{*}$ has always a well defined and invertible square root (being $\left.1+x_{1} x_{1}^{*}+x_{2} x_{2}^{*} \geq 1\right)$. Conversely, from $\alpha \alpha^{*}+\beta \beta^{*}+x^{2}=1$, it follows that $r^{2}=x_{1} x_{1}^{*}+x_{2} x_{2}^{*}=(1+x)(1-x)^{-1}$ is defined and is non-zero when the point $\left(\alpha, \alpha^{*}, \beta, \beta^{*}, x\right)$ we started from belongs to $S^{4} \backslash\{N, S\}$. The algebra extension of $\mathscr{B}\left(U_{N}\right)$ by $r^{-2}$ considered in LemmaC.3 geometrically corresponds indeed to the restriction to the points in the intersection of the charts $U_{N}$ and $U_{S}$ of $S^{4}$.
} 
Proof. Observe that $\phi_{N}\left(r^{2}\right)=\frac{1}{2}(1+x) c_{N}^{-2}$. Then, since $2 c_{S}^{2}=(1+x)$ (see (C.5) ), we immediately conclude that $\phi_{N}\left(r^{2}\right)=\phi_{N S}\left(r^{2}\right)$. For the inverse map set $\phi_{N S}^{-1}: c_{S}^{\mp 1} \mapsto \rho_{N}^{ \pm 1} r^{\mp 1}$.

The restriction maps characterizing the subsheaf of coinvariants $\mathscr{B} \simeq \mathscr{A}^{c o H}$ are the composition of the canonical inclusions $i_{N}: \mathscr{A}\left(U_{N}\right)^{c O H} \hookrightarrow \mathscr{A}\left(U_{N S}\right)^{c O H}$ and $i_{S}: \mathscr{A}\left(U_{S}\right)^{c O H} \hookrightarrow \mathscr{A}\left(U_{N S}\right)^{c O H}$ with the isomorphisms $\phi_{N}, \phi_{S}$ and $\phi_{N S}^{-1}$. These restriction maps are *-algebra homomorphisms and their explicit expression on the generators reads

$$
\begin{aligned}
r_{N, N S}^{\mathscr{B}}: \mathscr{B}\left(U_{N}\right) \longrightarrow \mathscr{B}\left(U_{N S}\right), \quad x_{i} \longmapsto x_{i}, \quad \rho_{N}^{ \pm 1} \longmapsto \rho_{N}^{ \pm 1}, \\
r_{S, N S}^{\mathscr{B}}: \mathscr{B}\left(U_{S}\right) \longrightarrow \mathscr{B}\left(U_{N S}\right), \quad y_{i} \longmapsto x_{i} r^{-2}, \quad \rho_{S}^{ \pm 1} \longmapsto \rho_{N}^{ \pm 1} r^{\mp 1} .
\end{aligned}
$$

The algebra of global sections $\mathscr{B}\left(S^{4}\right)$ is the pull-back

$$
\mathscr{B}\left(S^{4}\right)=\left\{\left(b_{N}, b_{S}\right) \in \mathscr{B}\left(U_{N}\right) \times \mathscr{B}\left(U_{S}\right) \mid r_{N, N S}^{\mathscr{B}}\left(b_{N}\right)=r_{S, N S}^{\mathscr{B}}\left(b_{S}\right)\right\} .
$$

The algebra $\mathscr{B}\left(S^{4}\right)$ is generated by the elements $\left(\rho_{N}^{-2} x_{i}, \rho_{S}^{-2} y_{i}\right),\left(\rho_{N}^{-2} x_{i}^{*}, \rho_{S}^{-2} y_{i}^{*}\right), i=1,2$ and $\left(1-2 \rho_{N}^{-2}, 2 \rho_{S}^{-2}-1\right)$ and is a copy of the coordinate algebra $O\left(S^{4}\right)=O\left(S^{7}\right)^{c o H}$.

Using the isomorphisms of Lemma C.2 and Lemma C.3 it is immediate to induce from $\mathscr{A}(U)^{\mathrm{coH}}$ the $K=O\left(\mathbb{T}^{2}\right)$-comodule structure on $\mathscr{B}(U),\left(U=U_{N}, U_{S}, U_{N S}\right)$ and to see that the restriction maps (C.16) are $K$-comodule maps. Considering the twist (3.31) on $K$ we then obtain the noncommutative algebra ${ }_{\sigma} \mathscr{B}\left(U_{N}\right)$ that is the (geometrically trivial central extension via the real elements $\rho_{N}^{ \pm}$of the) coordinate algebra on $\mathbb{R}_{\theta}^{4}$; i.e. the (exponential version of the Moyal-Weyl) algebra defined by the commutation relations $x_{1 \sigma} \bullet x_{2}=e^{-2 \pi i \theta} x_{2} \bullet x_{1}$. Similarly for ${ }_{\sigma} \mathscr{B}\left(U_{S}\right)$, and for ${ }_{\sigma} \mathscr{B}\left(U_{N S}\right)$ that is the geometrically nontrivial central extension of $\sigma \mathscr{B}\left(U_{N}\right)$ via the real elements $r^{ \pm 1}$. These algebras and the restriction maps $\Sigma\left(r_{N, N S}^{\mathscr{B}}\right)=r_{N, N S}^{\mathscr{B}}$, $\Sigma\left(r_{S, N S}^{\mathscr{B}}\right)=r_{S, N S}^{\mathscr{B}}$, define the sheaf ${ }_{\sigma} \mathscr{B}$ of noncommutative coordinates algebras over $S^{4}$, i.e. define $S_{\theta}^{4}$ as a ringed space.

\section{References}

[1] P. Aschieri and F. Bonechi, On the noncommutative geometry of twisted spheres, Lett. Math. Phys. 59 (2002) 133-156.

[2] P. Aschieri, M. Dimitrijevic, F. Meyer and J. Wess, Noncommutative geometry and gravity, Class. Quant. Grav. 23 (2006) 1883-1911.

[3] P. Aschieri and L. Castellani, R-matrix formulation of the quantum inhomogeneous groups $I S O_{q, r}(N)$ and $I S p_{q, r}(N)$, Lett. Math. Phys. 36 (1996) 197-211.

[4] P. Aschieri and A. Schenkel, Noncommutative connections on bimodules and Drinfeld twist deformation, Adv. Theor. Math. Phys. 18 (2014) 513-612.

[5] G. E. Barnes, A. Schenkel and R. J. Szabo, Nonassociative geometry in quasi-Hopf representation categories I: Bimodules and their internal homomorphisms, J. Geom. Phys. 89 (2014) 111-152.

[6] G. E. Barnes, A. Schenkel and R. J. Szabo, Nonassociative geometry in quasi-Hopf representation categories II: Connections and curvature, J. Geom. Phys. 106 (2016) 234-255. 
[7] P. Bieliavsky and V. Gayral, Deformation Quantization for Actions of Kählerian Lie Groups, Mem. Amer. Math. Soc. 236 (2015).

[8] S. Brain and G. Landi, Moduli spaces of non-commutative instantons: gauging away noncommutative parameters, Q. J. Math. 63 (2012) 41-86.

[9] S. Brain and S. Majid, Quantisation of twistor theory by cocycle twist, Commun. Math. Phys. 284 (2008) 713-774.

[10] T. Brzeziński, G. Janelidze and T. Maszczyk, Galois structures, in P.M. Hajac (Ed.) Lecture Notes on Noncommutative Geometry and Quantum Groups. Available at http://www . mimuw. edu.pl/ pwit/toknotes/toknotes.pdf

[11] T. Brzeziński and S. Majid, Quantum group gauge theory on quantum spaces, Commun. Math. Phys. 157 (1993) 591-638. Erratum 167 (1995) 235.

[12] L. S. Cirio and C. Pagani, A 4-Sphere with non-central radius and its instanton sheaf, Lett. Math. Phys. 105 (2015) 169-197.

[13] A. Connes and M. Dubois-Violette, Noncommutative finite-dimensional manifolds. I. Spherical manifolds and related examples, Commun. Math. Phys. 230 (2002) 539-579.

[14] A. Connes and G. Landi, Noncommutative manifolds: the instanton algebra and isospectral deformations, Commun. Math. Phys. 221 (2001) 141-159.

[15] L. Dąbrowski, H. Grosse and P.M. Hajac, Strong Connections and Chern-Connes Pairing in the Hopf-Galois Theory, Commun. Math. Phys. 220 (2001) 301-331.

[16] Y. Doi, Braided bialgebras and quadratic bialgebras, Comm. Algebra 21 (1993) 1731-1749.

[17] V. G. Drinfeld, On constant quasiclassical solutions of the Yang-Baxter quantum equation, Soviet Math. Dokl. 28 (1983) 667-671.

[18] V. G. Drinfeld, Hopf algebras and the quantum Yang-Baxter equation, Soviet Math. Dokl. 32 (1985) 254-258.

[19] M. Dubois-Violette and T. Masson, On the first order operators in bimodules, Lett. Math. Phys. 37 (1996) 467-474.

[20] C. Kassel, Quantum Groups, Springer-Verlag, New York (1995).

[21] A. Klimyk and K. Schmüdgen, Quantum groups and their representations, Springer-Verlag, Berlin Heidelberg (1997).

[22] H. F. Kreimer and M. Takeuchi, Hopf algebras and Galois extensions of an algebra, Indiana Univ. Math. J. 30 (1981) 675-692.

[23] G. Landi and W. van Suijlekom, Principal fibrations from noncommutative spheres, Commun. Math. Phys. 260 (2005) 203-225.

[24] G. Landi and W. van Suijlekom, Noncommutative instantons from twisted conformal symmetries, Commun. Math. Phys. 271 (2007) 591-634. 
[25] S. Majid, Foundations of quantum group theory, Cambridge, UK: Univ. Pr. (1995).

[26] S. Montgomery, Hopf algebras and their actions on rings, AMS (1993).

[27] S. Montgomery and H.J. Schneider, Krull relations in Hopf Galois extensions: lifting and twisting, J. Algebra 288 (2005) 364-383.

[28] J. Mourad, Linear connections in noncommutative geometry, Class. Quant. Grav. 12 (1995) 965-974.

[29] V. Ostrik, Module categories, weak Hopf algebras and modular invariants, Transform. Groups 8 (2003) 177-206.

[30] M. Pflaum, Quantum groups on fibre bundles, Commun. Math. Phys. 166 (1994) 279-315.

[31] N. Reshetikhin, Multiparameter quantum groups and twisted quasitriangular Hopf algebras, Lett. Math. Phys. 20 (1990) 331-335.

[32] M. Rieffel, Deformation quantization for actions of $R^{d}$, Mem. Amer. Math. Soc. 106 (1993) 506.

[33] P. Schauenburg and H.J. Schneider, On generalized Hopf Galois extensions, J. Pure App. Algebra 202 (2005) 168-194.

[34] H.J. Schneider, Principal homogeneous spaces for arbitrary Hopf algebras, Israel J. Math. 72 (1990) 167-195.

[35] J. Varilly, Quantum symmetry groups of noncommutative spheres, Commun. Math. Phys. 221 (2001) 511-523. 